\title{
Subvariedades isoparamétricas do espaço Euclidiano
}

\author{
Jaime Leonardo Orjuela Chamorro
}

\author{
DisSERTAÇÃO APRESENTADA \\ $\mathrm{AO}$ \\ Instituto DE Matemática e Estatística \\ DA \\ Universidade de SÃo Paulo \\ PARA \\ OBTENÇÃO DO TÍTULO \\ DE \\ Mestre em CiÊnCias
}

Área de Concentração: Matemática

Orientador: Prof. Dr. Claudio Gorodski

Durante o desenvolvimento deste trabalho o autor recebeu auxílio financeiro da CNPq

São Paulo, abril de 2008 


\title{
Subvariedades isoparamétricas do espaço Euclidiano
}

\author{
Este exemplar corresponde à redação \\ final da dissertação devidamente corrigida \\ e defendida por Jaime Leonardo Orjuela Chamorro \\ e aprovada pela Comissão Julgadora.
}

Banca Examinadora:

- Prof. Dr. Claudio Gorodski (orientador) - IME-USP.

- Prof. Dr. Marcos Martins Alexandrino da Silva - IME-USP.

- Prof. Dr. Ruy Tojeiro de Figueiredo Júnior - UFSCar. 
À Aurora e à Liz Angélica... 
"É preciso notar, no entanto, que, se Deus existe, se criou verdadeiramente a terra, fê-la, como se sabe, segundo a geometria de Euclides, e não deu ao espírito humano senão a noção das três dimensões do espaço.

Entretanto, encontraram-se, encontram-se ainda geômetras e filósofos, mesmo eminentes, para duvidar de que todo o universo e até mesmo todos os mundos tenham sido criados somente de acordo com os princípios

de Euclides. Ousam mesmo supor que duas paralelas que, de acordo com as leis de Euclides, jamais se poderão encontrar na terra, possam encontrar-se em alguma parte, no infinito."

Fiodor Dostoievski, Os irmãos Karamasov. 
Agradecimentos

Agradeço à Aurora, minha mãe, e ao resto da minha família pelo apoio desde a distância. A os meus amigos pela ajuda e estímulo. Agradeço ao professor Claudio Gorodski, pela paciência e ajuda na elaboração deste trabalho, ao professor Marcos Martins Alexandrino da Silva pela colaboração ao longo do curso de mestrado e ao professor Ruy Tojeiro de Figueiredo Júnior pelas observações e sugestões. 
Resumo

O presente trabalho tem por objeto fazer uma introdução ao estudo das subvariedades isoparamétricas do espaço Euclidiano. Começamos com uma introdução ao desenvolvimento histórico desses objetos. A seguir apresentamos os conceitos básicos da teoria de subvariedades de formas espaciais. Deduzimos as equações fundamentais de primeira e segunda ordem e demonstramos o teorema fundamental da teoria de subvariedades. Em seguida damos a definição de subvariedade isoparamétrica e desenvolvemos conceitos elementares para o caso do espaço Euclidiano como são normais de curvatura, grupo de Coxeter, câmera de Weyl e variedades paralelas e focais. Provamos dois teoremas referentes à decomposição de subvariedades isoparamétricas do espaço Euclidiano adaptando ferramentas usadas em [HL97] para ocaso de subvariedades isoparamétricas de espaços de Hilbert. Demonstramos o teorema da fatia e discutimos sobre subvariedades isoparamétricas desde o ponto de vista clássico, a saber, aplicações isoparamétricas. Concluímos com alguns exemplos: hipersuperfícies isoparamétricas da esfera e órbitas principais da ação adjunta de um grupo de Lie sobre a respectiva álgebra de Lie. 
$\begin{array}{lll}\text { Abstract } & \text { Abse }\end{array}$

The goal of this dissertation is to present an introduction to the study of isoparametric submanifolds of Euclidean space. We begin with an introduction to the history of the subject. Then we present the basic results of submanifold theory of space forms. We compute the fundamental equations of first and second order, and we prove the fundamental theorem of submanifold theory. Next, we define isoparametric submanifolds and discuss some basic constructions, as curvature normals, Coxeter groups, Weyl chambers and parallel and focal submanifolds. We prove two decomposition theorems about isoprametric submanifolds using techniques that we learnt from [HL97], paper in which the case of submanifolds of Hilbert spaces is studied. Then we prove slice theorem. We also discuss those submanifold from the classical point of view, namely, isoparametric maps. We finish by explaining some examples: isoparametric hipersurfaces of spheres and principal orbits of the adjoint action of a Lie group on its Lie algebra. 
Sumário

1 Preliminares $\quad 5$

1.1 Subvariedades de formas espaciais $\ldots \ldots \ldots \ldots \ldots$

1.1.1 Equações fundamentais de primeira ordem . . . . . . . . . . . . 8

1.1.2 Equações fundamentais de segunda ordem . . . . . . . . . . . . . . . . 10

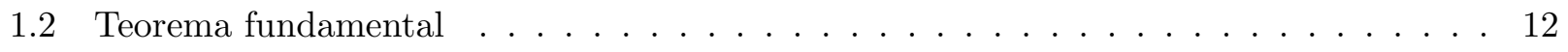

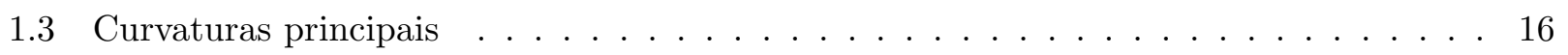

1.4 Subvariedades totalmente geodésicas e subvariedades totalmente umbílicas . . . . . . 17

1.4.1 Subvariedades totalmente geodésicas . . . . . . . . . . . . 17

1.4.2 Subvariedades totalmente umbílicas . . . . . . . . . . . . . . 17

1.5 Redução de codimensão . . . . . . . . . . . . . . . . . . 18

1.6 Submersões Riemannianas . . . . . . . . . . . . . . . . . . . . . . . 20

vii 
2 Subvariedades isoparamétricas $\quad 23$

2.1 Variedades focais e paralelas . . . . . . . . . . . . . . . . . . . 25

2.2 Normais de curvatura . . . . . . . . . . . . . . . . . . . . . . 29

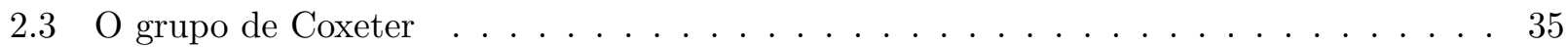

$2.3 .1 \quad$ A câmera de Weyl . . . . . . . . . . . . . . . . . . . . . . . 43

2.4 Decomposição de subvariedades isoparamétricas . . . . . . . . . . . . . . . . . . 44

2.5 Teorema da fatia . . . . . . . . . . . . . . . . . . . . . 49

2.6 Aplicações isoparamétricas . . . . . . . . . . . . . . . . . . . . . . 5 50

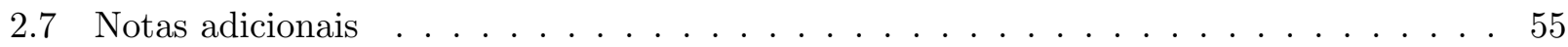

$\begin{array}{llc}3 & \text { Exemplos } & 57\end{array}$

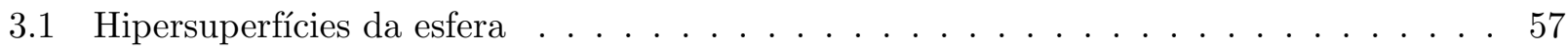

3.2 Órbitas da representação adjunta . . . . . . . . . . . . . . . . . . . 61

$\begin{array}{ll}\text { A Pontos críticos e subvariedades isoparamétricas } & 67\end{array}$

$\begin{array}{ll}\text { Referências bibliográficas } & 71\end{array}$

$\begin{array}{ll}\text { Índice Remissivo } & 72\end{array}$ 
Seja $f$ uma função real definida em uma variedade Riemanniana $\bar{M}$. O quadrado do comprimento do gradiente $|\operatorname{grad} f|^{2}$ e o Lapaciano $\Delta f$ de $f$ são chamados de primeiro e segundo parâmetro diferencial de $f$, respectivamente. O termo família isoparamétrica de hipersuperfícies de $\bar{M}$ foi introduzido por Levi-Civita, para o caso em que $\bar{M}=\mathbf{E}^{3}$ é o espaço Euclidiano tridimensional, em um artigo publicado em 1937, como sendo a coleção dos conjuntos de nível $N_{f}$ de uma função $f$ tal que o primeiro e o segundo parâmetro diferencial de $f$ são constantes ao longo de cada um dos elementos de $N_{f}$. Uma função com esta propriedade é chamada de função isoparamétrica. Ele estudou a classificação das famílias de superfícies isoparamétricas de $\mathbf{E}^{3}$ e obteve resultados que tinhan sido publicados por Somigliana em 1919 e B. Segre em 1924. Estes últimos realizaram seus trabalhos a respeito baseados em estudos de ótica geométrica feitos anteriormente por Laura.

Entre 1938 e 1940 É. Cartan publicou quatro artigos onde inicia o estudo da classificação das hipersuperfícies isoparamétricas de formas espaciais. Dentre alguns dos resultados obtidos por Cartan está a chamada fórmula fundamental de Cartan, a saber

$$
\sum_{j=1, j \neq i}^{g} m_{j} \frac{\kappa-\lambda_{i} \lambda_{j}}{\lambda_{i}-\lambda_{j}}=0
$$

onde $\lambda_{1}, \ldots, \lambda_{g}$ e $m_{1}, \ldots, m_{g}$ são as curvaturas principais e as respectivas multiplicidades de uma 
hipersuperfície isoparamétrica $M$ de uma forma espacial $\bar{M}$ com curvatura seccional $\kappa$. Cartan estudou também polinômios homogeneos $p$ definidos em $\mathbf{E}^{n+1}$ cujo primeiro parâmetro diferencial é constante na esfera $\mathbf{S}^{n}$ e com segundo parâmetro diferencial nulo (ou seja com $p$ harmônico). Em particular ele mostrou que uma família isoparamétrica de $\mathbf{S}^{n}$ com $g$ curvaturas principais, todas com a mesma multiplicidade é $N_{f}$, onde $f$ é a restrição à esfera de um polinômio homogeneo e harmônico $p$ satisfazendo

$$
|\operatorname{grad} p|^{2}=g^{2} r^{2(g-1)},
$$

sendo $r$ a função distância à origem de $\mathbf{E}^{n+1}$.

Só a partir de 1971 voltaram a aparecer artigos relacionados com os desenvolvimentos feitos por Cartan. Os primeiros depois permitiram fazer a classificação das hipersuperfícies isoparamétricas homogêneas da esfera. Em 1972 Takagi e Takahashi mostraram que para hipersuperfícies isoparamétricas homogêneas da esfera o número $g$ de curvaturas principais diferentes é $g=1,2,3,4,6$, eles também listaram as possibilidades para $m_{1}, \ldots, m_{g}$. Em 1973 Münzner escreveu dois artigos, que só foram publicados entre 1980 e 1981, nos quais estudou algumas propriedades geométricas e topológicas de hipersuperfícies isoparamétricas da esfera. Ele provou que as multiplicidades das curvaturas principais satisfazem a relação

$$
m_{i}=m_{i+2}
$$

com índices módulo $g$. Ele também generalizou os resultados de Cartan acerca de polinômios homogêneos. Mais precisamente ele mostrou que se $M \subset \mathbf{S}^{n}$ é isoparamétrica então $M \subset f^{-1}(c) \cap \mathbf{S}^{n}$ onde $f$ é um polinômio homogêneo satisfazendo

$$
|\operatorname{grad} f|^{2}=g^{2} r^{2(g-1)}
$$

e

$$
\Delta f=\frac{m_{2}-m_{1}}{2} g r^{g-2} .
$$

Tal vez o resultado mais importante obtido pelo Münzner foi a determinação do número $g$ de curvaturas principais de uma hipersuperfície isoparamétrica da esfera. Usando argumentos de topologia ele mostrou que $g \in\{1,2,3,4,6\}$.

Hão sido propostos varios conceitos que generalizam a noção de hipersuperfície isoparamétrica para codimensão maior do que um. A primeira definição do que agora é comummente chamado de 
subvariedade isoparamétrica é devida à generalização feita por Harle * da noção de função isoparamétrica para aplicações com valores vetoriais (vide seção 2.6). De acordo com sua definição ele verifucou que cada membro de uma família de subvariedades isoparamétricas de uma forma espacial tem fibrado normal raso ${ }^{\dagger}$ e os auto-valores do operador de forma são constantes ao longo de campos normais paralelos. Terng adotou esta como a definição de subvariedade isoparamétrica do espaço Euclidiano. Posteriormente esta ultima foi generalizada para subvariedades de espaços de Hilbert. Para uma apresentação mais ampla da história do conceito de subvariedade isoparamétrica ver [Tho00].

No presente trabalho faremos uma introdução ao estudo das subvariedades isoparamétricas do espaço Euclidiano na direção iniciada por Terng.

No primeiro capítulo apresentamos algumas noções básicas da teoria de subvariedades de formas espaciais. Em particular deduzimos as equações fundamentais de primeira e segunda ordem e damos uma demonstração do teorema fundamental (para o caso $\kappa=0$ ). Além disto introduzimos os conceitos de curvatura principal, subvariedades totalmente geodésicas e totalmente umbílicas . Fazemos a demonstração do teorema de redução de codimensão para caso do espaço Eulcidiano assim como o da esfera. Finalmente enunciamos e mostramos algumas propriedades das submersões Riemanianas.

No capítulo segundo introduzimos o conceito de subvariedades isoparamétricas do espaço Euclidiano e mostramos que subvariedades isoparamétricas da esfera também são isoparamétricas no espaço Euclidiano e viceversa. Introduzimos o conceito de variedades paralelas e focais e desenvolvemos algumas de suas principais propriedades, em particular deduzimos a chamada fórmula do tubo. Em seguida iniciamos o estudo de varios conceitos que nos darão informação geométrica das subvariedades isoparamétricas do espaço Euclidiano, entre otros as normais de curvatura e o grupo de Coxeter. Demonstramos o teorema de Terng que associa a cada subvariedade isoparamétrica compacta um grupo de Coxeter. Demonstraremos dois teoremas relativos à decomposição de subvariedades isoparamétricas. A saber, mostramos que uma subvariedade isoparamétrica completa do espaço Euclidiano decompõe-se em um produto de uma subvariedade isoparamétrica compacta e um factor Euclidiano e que é redutível se e somente se seu respectivo grupo de Coxeter é redutível. Para isto usamos a proposição (2.2.4). Em [PT88] e [BCO03] são usados o teorema fundamental e o lema de Moore para mostrar estes fatos, alem disto são obtidos apenas resultados locais. Nós adaptamos ferramenteas usadas em [HL97] para o caso de dimensão finita, tanto para a decomposição quanto para a proposição (2.2.4). Finalizamos este capítulo com a demonstração do teorema da fatia e com

\footnotetext{
* Seu trabalho passou desapersebido por muito tempo já que a primeira publicação foi feita em português.

†Do inglês: 'flat'.
} 
uma curta discussão sobre aplicações isoparamétricas.

No terceiro e último capítulo daremos alguns exemplos. Demostraremos o resultado de Münzner que nos diz como são exatamante as curvaturas principais de uma hipersuperfície compacta da esfera e como uma aplicação deste demonstraremos a fórmula fundamental de Cartan. Faremos notar que órbitas principais da ação adjunta de um grupo de Lie conexo compacto são isoparamétricas. 


\section{CAPÍtULO 1}

\section{Preliminares}

Seja $\phi: M \longrightarrow \bar{M}$ uma imersão, onde $\bar{M}$ é uma variedade Riemanniana. A equação

$$
\langle u, v\rangle_{p}=\left\langle d \phi_{p} u, d \phi_{p} v\right\rangle_{\phi(p)}
$$

para todo $p \in M$ e todo par $u, v \in T_{p} M$, define uma métrica Riemanniana sobre $M$ que faz com que $\phi$ seja uma isometria local de $M$ sobre $\phi(M)$. Em particular toda subvariedade imersa $M$ de um variedade Riemanniana $\bar{M}$ pode ser equipada, de maneira natural, de uma estrutura de variedade Riemanniana.

Pela forma local das imersões, existem cartas de $M$ e $\bar{M}$, nas quais a representação local de $\phi$ é uma inclusão. Como faremos apenas cálculos locais, podemos assumir de antemão, sem perder generalidade, que $\phi$ é uma inclusão. A inclusão será denotada por $\iota$.

É possível generalizar as idéias utilizadas no estudo da geometria das superfícies do espaço Euclidiano $\mathbf{E}^{3}$ ao caso das subvariedades imersas de espaços de formas. Neste capítulo introduzimos os conceitos básicos da teoria de subvariedades de variedades Riemannianas assim como as equações fundamentais das subvariedades de formas espaciais. Também enunciaremos o Teorema fundamental 
de subvariedades de formas espaciais.

Neste capítulo $\operatorname{dim} \bar{M}=n$ e $\operatorname{dim} M=m$.

\subsection{Subvariedades de formas espaciais}

Sejam $\bar{M}$ uma variedade Riemanniana e $M$ uma subvariedade de $\bar{M}$. Pela forma local das imersões podemos considerar cartas locais $\mathbf{x}: \mathcal{A} \longrightarrow \mathbf{R}^{m}$ e $\overline{\mathbf{x}}: \overline{\mathcal{A}} \longrightarrow \mathbf{R}^{n}$ de $M$ e $\bar{M}$, respectivamente, tais que $\iota(\mathcal{A}) \subset \overline{\mathcal{A}} \mathrm{e}$

$$
\iota(p)=\overline{\mathbf{x}}^{-1}\left(\mathbf{x}_{1}(p), \ldots, \mathbf{x}_{m}(p), 0, \ldots, 0\right)
$$

para todo $p \in \mathcal{A}$. Sejam $\mathbf{e}_{1}, \ldots, \mathbf{e}_{n}$, campos locais dados por

$$
\mathbf{e}_{i}(p)=\left.\frac{\partial}{\partial \overline{\mathbf{x}}_{i}}\right|_{\iota(p)},
$$

para todo $p \in \mathcal{A}$ e cada $i=1, \ldots, n$. Então $\left\{\mathbf{e}_{1}(p), \ldots, \mathbf{e}_{n}(p)\right\}$ é uma base de $T_{p} \bar{M}$, para cada $p \in \mathcal{A}$.

Consideremos o fibrado tangente de $\bar{M}$

$$
\tau: T \bar{M} \longrightarrow \bar{M}
$$

Sejam $F=\tau^{-1}(M)$ e $\varphi=\left.\tau\right|_{F}$. Então $\varphi: F \longrightarrow M$ é um fibrado vetorial sobre $M$ tal que $F_{p}=T \bar{M}_{p}$ para todo $p \in M$. Com efeito, definindo $t: \varphi^{-1}(\mathcal{A}) \longrightarrow \mathcal{A} \times \mathbf{R}^{n}$ por $t(u)=\left(p,\left(a_{1}, \ldots, a_{n}\right)\right)$, para todo $u=\sum_{i} a_{i} \mathbf{e}_{i}(p) \in \varphi^{-1}(\mathcal{A})$, vemos que $t$ é uma trivialização local de $(\varphi, F, M)$.

Consideremos o referencial local de $F$, construído acima. Observemos que os primeiros $m$ campos constituem um referencial local de $T M$. Aplicando o processo de ortogonalização de Gram-Schmidt, construímos um referencial ortogonal local de $F$ tal que os primeiros $m$ campos continuam sendo um referencial local de $T M$. Assim para cada ponto de $M$ existem $n-m$ seções de $F$, definidas em uma vizinhança do ponto em $M$, que constituem uma base para o complemento ortogonal do espaço tangente, dos pontos desta vizinhança, nas respectivas fibras de $F$. Desta maneira observamos que os complementos ortogonais das fibras de $T M$ nas fibras correspondentes de $F$ constituem um fibrado vetorial sobre $M$. Este é denotado por $\nu M$ e dizemos que $\nu M$ é o fibrado normal de $M$. 
Normalmente $F$ é denotado por $\left.T \bar{M}\right|_{M}$. Tem-se a seguinte decomposição ortogonal

$$
\left.T \bar{M}\right|_{M}=T M \oplus \nu M
$$

Dada uma seção $U \in \Gamma(F) ; U^{\top}$ e $U^{\perp}$ denotarão as projeções tangencial e normal, induzidas pela métrica de $\bar{M}$, de $U$ sobre $\Gamma(T M)$ e $\Gamma(\nu M)$ respectivamente.

Seja $\bar{\nabla}$ a conexão de Levi-Civita de $\bar{M}$. Se $X$ e $U$ seções de $T M$ e $F$ respectivamente, podemos estendé-las localmente a campos $\bar{X}$ e $\bar{U}$ de $T \bar{M}$ (por exemplo, usando a forma local das imersões

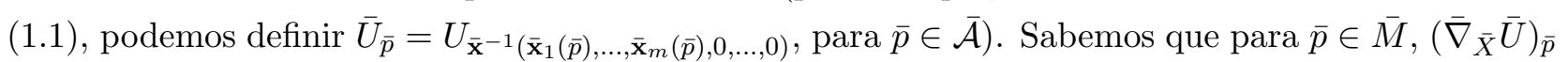
só depende de $\bar{X}_{\bar{p}}$ e da restrição de $\bar{U}$ a uma curva $\gamma:(-\epsilon, \epsilon) \longrightarrow \bar{M} \operatorname{com} \gamma(0)=\bar{p}$ e $\gamma^{\prime}(0)=\bar{X}_{\bar{p}}$. Em particular, para pontos em $M$, podemos escolher curvas em $M$. Desta maneira podemos definir $\bar{\nabla}_{X} U$ sem ambigüidade por

$$
\left(\bar{\nabla}_{X} U\right)_{p}=\left(\bar{\nabla}_{\bar{X}} \bar{U}\right)_{p}
$$

onde $\bar{X}$ e $\bar{U}$ são extensões de $X$ e $U$, respectivamente, definidos em uma vizinhança de $p$ em $\bar{M}$, para todo $p \in M$.

Observação 1.1.1. Na verdade a equação acima define uma conexão sobre $F$, que por abuso de notação denotaremos com $\bar{\nabla}$, tal que

$$
X\langle U, V\rangle=\left\langle\bar{\nabla}_{X} U, V\right\rangle+\left\langle U, \bar{\nabla}_{X} V\right\rangle
$$

para seções $X \in \Gamma(T M)$ e $U, V \in \Gamma(F)$. Nós utilizaremos com freqüência esta propriedade.

Sejam $[|\cdot, \cdot|]$ e $[\cdot, \cdot]$ os colchetes de Lie de $\bar{M}$ e $M$, respectivamente. Se $X, Y \in \Gamma(T M)$ então

$$
\bar{\nabla}_{X} Y-\bar{\nabla}_{Y} X=[X, Y]
$$

isto segue da definição de $\bar{\nabla}_{X} Y$ e do fato seguinte. Se $\bar{X}$ e $\bar{Y}$ são extensões locais de $X$ e $Y$, respectivamente, temos que $d \iota X=\bar{X}_{\iota}$ e $d \iota Y=\bar{Y}_{\iota}$, e portanto $d \iota[X, Y]=[|\bar{X}, \bar{Y}|]_{\iota}$ (vide [Spi70]).

De outro lado, seja $\bar{R}$ o tensor de curvatura de $\bar{M}$. Dados $\bar{X}, \bar{Y}, \bar{Z} \in \Gamma(T \bar{M})$ e $p \in \bar{M}$

$$
(\bar{R}(\bar{X}, \bar{Y}) \bar{Z})_{p}=\left(\left(\bar{\nabla}_{\bar{X}} \bar{\nabla}_{\bar{Y}}-\bar{\nabla}_{\bar{Y}} \bar{\nabla}_{\bar{X}}-\bar{\nabla}_{[|\bar{X}, \bar{Y}|]}\right) Z\right)_{p}
$$


só depende de $\bar{X}_{p}, \bar{Y}_{p}$ e $\bar{Z}_{p}$. Assim para seções $X, Y \in \Gamma(T M)$ e $U \in \Gamma(F)$ tem-se

$$
\bar{R}(X, Y) U=\left(\bar{\nabla}_{X} \bar{\nabla}_{Y}-\bar{\nabla}_{Y} \bar{\nabla}_{X}-\bar{\nabla}_{[X, Y]}\right) U
$$

\subsubsection{Equações fundamentais de primeira ordem}

Dada uma subvariedade $M$ de uma variedade Riemanniana $\bar{M}$. A métrica de $\bar{M}$ induz a decomposição ortogonal

$$
T_{p} \bar{M}=T_{p} M \oplus \nu_{p} M
$$

para todo $p \in M$, onde $\nu_{p} M$ denota a fibra de $\nu M$ em $p$, a qual é chamada o espaço normal de $M$ em $p$. As seções de $\nu M$ diz-sem-se campos vetoriais normais ou simplesmente campos normais.

A segunda forma fundamental $\alpha$ e o operador de forma $A$, de $M$, são definidos por

$$
\alpha(X, Y)=\left(\bar{\nabla}_{X} Y\right)^{\perp}
$$

e

$$
A_{\xi} X=-\left(\bar{\nabla}_{X} \xi\right)^{\top}
$$

para $X, Y \in \Gamma(T M)$ e $\xi \in \Gamma(\nu M)$. Claramente $A$ e $\alpha$ são R-bilineares. Fixando um campo normal $\xi \in \nu M, A_{\xi}$ diz-se operador de forma de $M$ na direção de $\xi$.

Agora, $\left\langle\bar{\nabla}_{X} Y, \xi\right\rangle=X\langle Y, \xi\rangle-\left\langle Y, \bar{\nabla}_{X} \xi\right\rangle=\left\langle-\bar{\nabla}_{X} \xi, Y\right\rangle$, logo a segunda forma fundamental e o operador de forma de $M$ estão relacionados por

$$
\langle\alpha(X, Y), \xi\rangle=\left\langle A_{\xi} X, Y\right\rangle
$$

Sabemos que, para $p \in M, \alpha(X, Y)_{p}$ depende de $X_{p}$ e da restrição de $\mathrm{Y}$ a uma curva $\gamma:(-\epsilon, \epsilon) \longrightarrow M$, com $\gamma(0)=p$ e $\gamma^{\prime}(0)=Y_{p}$. Dado $Y^{\prime} \in \Gamma(T M)$ tem-se $\left\langle\alpha(X, Y)-\alpha\left(X, Y^{\prime}\right), \xi\right\rangle=\left\langle A_{\xi} X, Y-Y^{\prime}\right\rangle$. Então se $p \in M \operatorname{com} Y_{p}=Y_{p}^{\prime}$, obtemos $\alpha(X, Y)_{p}=\alpha\left(X, Y^{\prime}\right)_{p}$. Portanto para todo $p \in M \alpha(X, Y)_{p}$ só depende de $X_{p}$ e $Y_{p}$. Analogamente, usando (1.2), vemos que $\left(A_{\xi} X\right)_{p}$ depende somente de $X_{p}$ e 
$\xi_{p}$. Assim, na verdade

$$
\alpha: \Gamma(T M) \times \Gamma(T M) \longrightarrow \Gamma(\nu M) \text { e } A: \Gamma(T M) \times \Gamma(\nu M) \longrightarrow \Gamma(T M)
$$

são tensores.

Sejam $X, Y$ e $Z$ seções de $T M$ e $f: M \longrightarrow \mathbf{R}$ uma função suave. Temos que

$$
\left(\bar{\nabla}_{f X} Y\right)^{\top}=\left(f \bar{\nabla}_{X} Y\right)^{\top}=f\left(\bar{\nabla}_{X} Y\right)^{\top}
$$

e

$$
\left(\bar{\nabla}_{X} f Y\right)^{\top}=\left(X(f) Y+\bar{\nabla}_{X} Y\right)^{\top}=X(f) Y+\left(\bar{\nabla}_{X} Y\right)^{\top}
$$

De outro lado

$$
X\langle Y, Z\rangle=\left\langle\bar{\nabla}_{X} Y, Z\right\rangle+\left\langle X, \bar{\nabla}_{X} Z\right\rangle=\left\langle\left(\bar{\nabla}_{X} Y\right)^{\top}, Z\right\rangle+\left\langle Y,\left(\bar{\nabla}_{X} Z\right)^{\top}\right\rangle
$$

e

$$
\left(\bar{\nabla}_{X} Y\right)^{\top}-\left(\bar{\nabla}_{Y} X\right)^{\top}=\left(\bar{\nabla}_{X} Y-\bar{\nabla}_{Y} X\right)^{\top}=[X, Y]^{\top}=[X, Y] .
$$

Portanto a conexão de Levi-Civita $\nabla$ de $M$ esta dada por $\nabla_{X} Y=\left(\bar{\nabla}_{X} Y\right)^{\top}$. Assim obtemos a fórmula de Gauss

$$
\bar{\nabla}_{X} Y=\nabla_{X} Y+\alpha(X, Y) .
$$

Da fórmula de Gauss e do fato de $\nabla$ ter torção nula, segue que

$$
\alpha(X, Y)-\alpha(Y, X)=\left(\bar{\nabla}_{X} Y-\bar{\nabla}_{Y} X\right)-[X, Y]=0
$$

portanto $\alpha$ é um tensor simétrico. A simetria de $\alpha$ e a equação (1.2) mostram que $A_{v}$ é um operador auto-adjunto de $T_{p} M$ para todo $v \in \nu_{p} M$ e todo $p \in M$.

Se $X \in \Gamma(T M), \xi \in \Gamma(\nu M)$ e $f: M \longrightarrow \mathbf{R}$ é uma função suave, então

$$
\left(\bar{\nabla}_{f X} \xi\right)^{\perp}=\left(f \bar{\nabla}_{X} \xi\right)^{\perp}=f\left(\bar{\nabla}_{X} \xi\right)^{\perp}
$$

e

$$
\left(\bar{\nabla}_{X} f \xi\right)^{\perp}=\left(X(f) \xi+f \bar{\nabla}_{X} \xi\right)^{\perp}=X(f) \xi+\left(\bar{\nabla}_{X} \xi\right)^{\perp}
$$


Assim a projeção normal de $\bar{\nabla}_{X} \xi$ define uma conexão sobre $\nu M$ chamada conexão normal, que é denotada por $\nabla^{\perp}$. Desta maneira obtemos a fórmula de Weingarten

$$
\bar{\nabla}_{X} \xi=-A_{\xi} X+\nabla_{X}^{\perp} \xi
$$

As fórmulas de Gauss e de Weingarten são as equações fundamentais de primeira ordem de $M$.

\subsubsection{Equações fundamentais de segunda ordem}

As derivadas covariantes da segunda forma fundamental e do operador de forma de $M$ estão dadas por

$$
\left(\nabla_{X}^{\perp} \alpha\right)(Y, Z)=\nabla_{X}^{\perp} \alpha(Y, Z)-\alpha\left(\nabla_{X} Y, Z\right)-\alpha\left(Y, \nabla_{X} Z\right)
$$

e

$$
\left(\nabla_{X} A\right)_{\xi} Y=\left(\nabla_{X} A_{\xi}\right) Y-A_{\nabla_{X}} \xi=\nabla_{X}\left(A_{\xi}\right) Y-A_{\xi}\left(\nabla_{X} Y\right)-A_{\nabla_{X} \xi} Y
$$

para $X, Y, Z \in \Gamma(T M)$ e $\xi \in \Gamma(\nu M)$. Temos uma relação entre as derivadas covariantes de $\alpha$ e $A$, no mesmo sentido de $(1.2)$

$$
\left\langle\left(\nabla_{X}^{\perp} \alpha\right)(Y, Z), \xi\right\rangle=\left\langle\left(\nabla_{X} A\right)_{\xi} Y, Z\right\rangle .
$$

Agora, sejam $\bar{R}$ e $R$ os tensores de curvatura de $\bar{M}$ e $M$, respectivamente, considerados com sinais como na observação (1.1.1). Temos que

$$
\begin{aligned}
\bar{R}(X, Y) Z & =R(X, Y) Z-A_{\alpha(Y, Z)} X+A_{\alpha(X, Z)} Y+ \\
& +\left(\nabla_{X}^{\perp} \alpha\right)(Y, Z)-\left(\nabla_{Y}^{\perp} \alpha\right)(X, Z) .
\end{aligned}
$$

Suponhamos que $\bar{M}$ é uma forma espacial e seja $\kappa$ sua curvatura seccional. Então a componente tangencial da equação acima é

$$
\begin{aligned}
\kappa(\langle Y, Z\rangle X-\langle X, Z\rangle Y) & =(\bar{R}(X, Y) Z)^{\top} \\
& =R(X, Y) Z-A_{\alpha(Y, Z)} X+A_{\alpha(X, Z)} Y
\end{aligned}
$$


e a componente normal da mesma é

$$
\begin{aligned}
0 & =(\bar{R}(X, Y) Z)^{\perp} \\
& =\left(\nabla_{X}^{\perp} \alpha\right)(Y, Z)-\left(\nabla_{Y}^{\perp} \alpha\right)(X, Z) .
\end{aligned}
$$

Se $W \in \Gamma(T M)$, as anteriores equações podem ser escritas assim

$$
\begin{aligned}
\langle R(X, Y) Z, W\rangle & =\kappa(\langle Y, Z\rangle\langle X, W\rangle-\langle X, Z\rangle\langle Y, W\rangle) \\
& +\langle\alpha(Y, Z), \alpha(X, W)\rangle-\langle\alpha(X, Z), \alpha(Y, W)\rangle
\end{aligned}
$$

e

$$
\left(\nabla_{X}^{\perp} \alpha\right)(Y, Z)=\left(\nabla_{Y}^{\perp} \alpha\right)(X, Z) .
$$

A primeira é chamada equação de Gauss e a segunda equação de Codazzi. De (1.3) a equação de Codazzi é equivalente a

$$
\left\langle\left(\nabla_{X} A\right)_{\xi} Y, Z\right\rangle=\left\langle\left(\nabla_{Y} A\right)_{\xi} X, Z\right\rangle .
$$

O tensor de curvatura do fibrado normal é de notado por $R^{\perp}$ e definido por

$$
R^{\perp}(X, Y) \xi=\nabla_{X}^{\perp} \nabla_{Y}^{\perp} \xi-\nabla_{Y}^{\perp} \nabla_{X}^{\perp} \xi-\nabla_{[X, Y]}^{\perp} \xi
$$

Usando as fórmulas de Gauss e Weingarten obtemos

$$
\begin{aligned}
0 & =\bar{R}(X, Y) \xi \\
& =\left(\nabla_{X} A\right)_{\xi} Y-\left(\nabla_{Y} A\right)_{\xi} X+R^{\perp}(X, Y) \xi+\alpha\left(A_{\xi} X, Y\right)-\alpha\left(X, A_{\xi} Y\right) .
\end{aligned}
$$

A componente tangencial desta última equação é a equação de Codazzi, e a componente normal é chamada equação de Ricci. Usando (1.2), a equação de Ricci pode ser escrita assim

$$
\left\langle R^{\perp}(X, Y) \xi, \eta\right\rangle=\left\langle\left[A_{\xi}, A_{\eta}\right] X, Y\right\rangle
$$

onde $\eta \in \Gamma(\nu M)$ e $\left[A_{\xi}, A_{\eta}\right]=A_{\xi} A_{\eta}-A_{\eta} A_{\xi}$. Se $R^{\perp} \equiv 0$ dizemos que $M$ tem fibrado normal raso. A interpretação geométrica do fibrado normal raso é que o transporte paralelo, em relação a $\nabla^{\perp}$, de vetores normais ao longo de curvas (em $M$ ) com mesmos pontos extremos só depende da classe 
de homotopia das curvas. Note que da equação de Ricci, o tensor de curvatura normal $R^{\perp}$ mede a não-comutatividade dos operadores de forma de $M$.

Observação 1.1.2. Pela simetria de $\alpha$ temos que $\left(\nabla_{X}^{\perp} \alpha\right)(Y, Z)=\left(\nabla_{X}^{\perp} \alpha\right)(Z, Y)$ assim na verdade a equação de Codazzi diz que a expressão $\left\langle\left(\nabla_{X} A\right)_{\xi} Y, Z\right\rangle$ é simétrica nas três variáveis tangenciais, ou seja

$$
\left\langle\left(\nabla_{X} A\right)_{\xi} Y, Z\right\rangle=\left\langle\left(\nabla_{Y} A\right)_{\xi} X, Z\right\rangle=\left\langle\left(\nabla_{Z} A\right)_{\xi} Y, X\right\rangle
$$

As equações de Gauss, Codazzi e Ricci são as equações fundamentais de segunda ordem de $M$.

\subsection{Teorema fundamental de subvariedades de formas espaciais}

Na seção anterior vimos que em uma subvariedade de uma forma espacial as equações de Gauss Codazzi e Ricci são satisfeitas. Localmente uma subvariedade de uma forma espacial fica completamente determinada pela conexão normal, a segunda forma fundamental e o operador de forma mediante estas equações. Mais precisamente tem-se o seguinte resultado.

Teorema 1.2.1. Sejam $M$ uma variedade Riemanniana de dimensão $m, \nu$ um fibrado vetorial Riemanniano sobre $M$ de posto $k, \nabla^{\nu}$ uma conexão métrica sobre $\nu$ e $\alpha$ um tensor simétrico sobre $M$ com valores em $\nu$. Definimos $A: \nu \longrightarrow \operatorname{End}(T M)$ por $\left\langle A_{v} u, w\right\rangle=\langle\alpha(u, w), v\rangle$, para $u, w \in T_{p} M$ e $v \in \nu_{p}, p \in M$. Suponhamos que $\alpha, A$ e $\nabla^{\nu}$ satisfazem as equações de Gauss, Codazzi e Ricci para algum número real $\kappa$. Então para cada ponto $p \in M$ existe uma vizinhança aberta $\mathcal{A}$ de $p$ em $M$ e uma imersão isométrica $\phi$ de $\mathcal{A}$ em uma forma espacial $\bar{M}$, de dimensão $n=m+k$ e curvatura seccional constante $\kappa$; tais que $\alpha$ e A são a segunda forma fundamental e o operador de forma de $\phi$, respectivamente e $\nu$ é isomorfo ao fibrado normal de $\phi$. A imersão $\phi$ é única salvo uma isometria de $\bar{M}$. Ainda mais, suponhamos que existem duas imersões isométricas $\psi_{1}, \psi_{2}: M \longrightarrow \bar{M}$ e um isomorfismo de fibrados vetoriais $\Psi: \nu_{1} M \longrightarrow \nu_{2} M$ tal que

$$
\begin{gathered}
\langle\Psi(\xi), \Psi(\eta)\rangle=\langle\xi, \eta\rangle, \\
\Psi\left(\alpha_{1}(X, Y)\right)=\alpha_{2}(X, Y)
\end{gathered}
$$

$e$

$$
\Psi\left(\nabla_{X}^{\perp_{1}} \xi\right)=\nabla_{X}^{\perp_{2}} \Psi(\xi)
$$


onde $\alpha_{i}$ e $\nabla^{\perp_{i}}$ são a segunda forma fundamental e a conexão normal de $\psi_{i}, i=1,2$; para todo par $X, Y \in \Gamma(T M)$ e todo par $\xi, \eta \in \Gamma\left(\nu_{1} M\right)$. Então existe uma isometria $B$ do espaço ambiente $\bar{M}$, tal que localmente $\psi_{2}=B \circ \psi_{1}$ e d $\left.B\right|_{\nu_{1} M}=\Psi$.

Demonstração. Daremos uma prova para o caso $\kappa=0$.

Consideremos o fibrado $F=T M \oplus \nu$. Definimos a conexão $\hat{\nabla}$ sobre $F$ por

$$
\hat{\nabla}_{X} Y=\nabla_{X} Y+\alpha(X, Y)
$$

$\mathrm{e}$

$$
\hat{\nabla}_{X} \xi=-A_{\xi} X+\nabla_{X}^{\nu} \xi
$$

para seções $X, Y \in \Gamma(T M)$ e $\xi \in \Gamma(\nu)$. As equações de Gauss, Codazzi e Ricci implicam que $F$ tem tensor de curvatura nulo. Logo, dado $p \in M$ existe um referencial ortonormal paralelo $U_{1}, \ldots, U_{n}$ de $F$, definido em uma vizinhança aberta $\mathcal{A}^{\prime}$ de $p$ em $M$. Seja $\omega_{1}, \ldots, \omega_{n}$ seu referencial dual. Temos que

$$
\begin{aligned}
d \omega_{i}(X, Y) & =X\left\langle U_{i}, Y\right\rangle-Y\left\langle U_{i}, X\right\rangle-\left\langle U_{i},[X, Y]\right\rangle \\
& =\left\langle U_{i}, \nabla_{X} Y+\alpha(X, Y)\right\rangle-\left\langle U_{i}, \nabla_{Y} X+\alpha(Y, X)\right\rangle-\left\langle U_{i},[X, Y]\right\rangle \\
& =0,
\end{aligned}
$$

para todo $i \in\{1, \ldots, n\}$ e cada par de seções $X, Y \in \Gamma(T M)$. Segue que as 1 -formas $\omega_{1} \ldots, \omega_{n}$ são fechadas, logo existem vizinhanças abertas conexas e simplesmente conexas $\mathcal{A}_{i}$ de $p$ em $\mathrm{M}$ e funções $\phi_{i}: \mathcal{A}_{i} \longrightarrow \mathbf{R}$ tais que $\omega_{i}=d \phi_{i}$, para $i \in\{1, \ldots, n\}$. Fazendo $\mathcal{A}=\cap_{i=1}^{n} \mathcal{A}_{i}$,

$$
\phi=\left(\phi_{1}, \ldots, \phi_{n}\right): \mathcal{A} \longrightarrow \mathbf{E}^{n}
$$

é uma imersão isométrica. Com efeito, Dadas seções $X, Y \in \Gamma(T M)$ temos que

$$
\langle X, Y\rangle=\sum_{i=1}^{n}\left\langle U_{i}, X\right\rangle\left\langle U_{i}, Y\right\rangle
$$


Se $\left(x_{1}, \ldots, x_{n}\right)$ são as coordenadas usuais de $\mathbf{E}^{n}$ então $d \phi(X)=\sum_{i=1}^{n} d \phi_{i}(X) \frac{\partial}{\partial x_{i}}, \operatorname{logo}$

$$
\begin{aligned}
\langle d \phi(X), d \phi(Y)\rangle & =\sum_{i, j=1}^{n} d \phi_{i}(X) d \phi_{j}(Y)\left\langle\frac{\partial}{\partial x_{i}}, \frac{\partial}{\partial x_{j}}\right\rangle \\
& =\sum_{i=1}^{n} d \phi_{i}(X) d \phi_{i}(Y) \\
& =\sum_{i=1}^{n} \omega_{i}(X) \omega_{i}(Y) \\
& =\sum_{i=1}^{n}\left\langle U_{i}, X\right\rangle\left\langle U_{i}, Y\right\rangle \\
& =\langle X, Y\rangle .
\end{aligned}
$$

Note que o referencial $U_{1}, \ldots, U_{n}$ é único salvo uma isometria linear de $\mathbf{E}^{n}$ e $\omega_{i}=d\left(\phi_{i}+a_{i}\right)$, para todo número real $a_{i}, i \in\{1, \ldots, n\}$. Assim a aplicação $\phi$ construída desta maneira é única salvo uma isometria de $\mathbf{E}^{n}$. Definindo $\tilde{\phi}:\left.\left.F\right|_{\mathcal{A}} \longrightarrow T \mathbf{E}^{n}\right|_{\mathcal{A}}$ por

$$
\tilde{\phi}\left(U_{i}\right)=\frac{\partial}{\partial x_{i}}
$$

para $X \in \Gamma(T M)$ temos que

$$
\begin{aligned}
\tilde{\phi}(X) & =\sum_{i=1}^{n}\left\langle U_{i}, X\right\rangle \hat{\phi}\left(U_{i}\right) \\
& =\sum_{i=1}^{n} \omega_{i}(X) \frac{\partial}{\partial x_{i}} \\
& =\sum_{i=1}^{n} d \phi_{i}(X) \frac{\partial}{\partial x_{i}} \\
& =d \phi(X) .
\end{aligned}
$$

Portanto $d \phi=\left.\tilde{\phi}\right|_{\left.T M\right|_{\mathcal{A}}}$. Agora, $\tilde{\phi}$ é uma isometria em cada fibra, $\operatorname{logo} \tilde{\phi}\left(\left.T M\right|_{\mathcal{A}}\right)$ e isomorfo a $T \mathcal{A}$ e $\tilde{\phi}\left(\left.\nu\right|_{\mathcal{A}}\right)$ é isomorfo a $\nu \mathcal{A}$. Ainda, como $\tilde{\phi}$ leva o referencial ortonormal paralelo $U_{1}, \ldots, U_{n}$ no 
referencial ortonormal paralelo $\frac{\partial}{\partial x_{1}}, \ldots, \frac{\partial}{\partial x_{n}}$ temos que

$$
\tilde{\phi}\left(\hat{\nabla}_{X} Y\right)=\bar{\nabla}_{d \phi(X)} \tilde{\phi}(Y) \text { e } \tilde{\phi}\left(\hat{\nabla}_{X} \xi\right)=\bar{\nabla}_{d \phi(X)} \tilde{\phi}(\xi)
$$

onde $\bar{\nabla}$ é a conexão de Levi-Civita de $\mathbf{E}^{n}$. Portanto $A, \alpha$ e $\nabla^{\nu}$ são o operador de forma a segunda forma fundamental e a conexão normal de $\phi$.

Agora, como o segundo resultado a ser provado também é local, sem perder generalidade, podemos supor $M$ conexa e $\psi_{1}$ e $\psi_{2}$ mergulhos isométricos. Seja

$$
\Phi: \psi_{1}^{*} T M=d \psi_{1}(T M) \oplus \nu_{1} M \longrightarrow d \psi_{2}(T M) \oplus \nu_{2} M=\psi_{2}^{*} T M
$$

dada por $\Phi(X)=X$ e $\Phi(\xi)=\Psi(\xi)$, para todo $X \in \Gamma(T M)$ e todo $\xi \in \Gamma\left(\nu_{1} M\right)$. Então por (1.4) $\Phi$ é um isomorfismo de fibrados vetoriais que preserva a métrica; ainda mais das equações (1.4), (1.5) e (1.6), para todo par $X, Y \in \Gamma(T M)$ e todo $\xi \in \Gamma\left(\nu_{1} M\right)$, tem-se

$$
\Phi\left(\hat{\nabla}_{X}^{1} Y\right)=\nabla_{X} Y+\Psi\left(\alpha_{1}(X, Y)\right)=\nabla_{X} Y+\alpha_{2}(X, Y)=\hat{\nabla}_{X}^{2} Y
$$

$\mathrm{e}$

$$
\begin{aligned}
\Phi\left(\hat{\nabla}_{X}^{1} Y\right) & =-A_{\xi}^{1} X+\Psi\left(\hat{\nabla}_{X}^{1} \xi\right) \\
& =-A_{\Psi(\xi)}^{2} X+\nabla^{\perp_{2}} \Psi(\xi) \\
& =\hat{\nabla}_{X}^{2} \Psi(\xi),
\end{aligned}
$$

onde $A^{i}$ e o operador de forma de $\psi_{i}$ e $\hat{\nabla}^{i}$ é definida como nas equações (1.7) e (1.8), para $i=1,2$. Pela primeira parte da prova sabemos que $\hat{\nabla}^{1}$ e $\hat{\nabla}^{2}$ têm tensor de curvatura nulo, assim $\Phi$ leva referenciais ortonormais paralelos em referenciais normais paralelos. Identificando $\psi_{1}^{*} T M$ e $\psi_{2}^{*} T M$ com $M \times \mathbf{E}^{n}, \Phi$ leva campos ortonormais constantes em campos ortonormais constantes, logo é da forma $(p, u) \mapsto\left(p, B_{0} u\right)$, onde $B_{0}$ é uma isometria linear de $\mathbf{E}^{n}$. Portanto

$$
\left.d B_{0}\right|_{\left(\nu_{1}\right)_{p} M}=\Psi_{p}
$$

$\mathrm{e}$

$$
B_{0}\left(d \psi_{1}\right)_{p}=\left(d \psi_{2}\right)_{p},
$$


para todo $p \in M$. Em particular $d\left(B_{0} \circ \psi_{1}-\psi_{2}\right)=0$ e como $M$ é conexa $B_{0} \circ \psi_{1}-\psi_{2}=-b$ para algum $b \in \mathbf{E}^{n}$. Logo

$$
\psi_{2}=B \circ \psi_{1},
$$

onde $B$ é a isometria de $\mathbf{E}^{n}$ dada por $B(x)=B_{0} x+b, x \in \mathbf{E}^{n}$.

\subsection{Curvaturas principais}

Seja $M$ uma subvariedade de uma forma espacial $\bar{M}$. Dados $p \in M$ e $v \in \nu_{p} M$ sabemos que $A_{v}$ é um operador auto-adjunto de $T_{p} M$. Os auto-valores, auto-vetores e auto-espaços de $A_{v}$ são chamados curvaturas principais, vetores de curvatura e espaços de curvaturas principais de $M$ na direção de $v$, respectivamente.

Observação 1.3.1. Como as curvaturas principais são as raízes de um polinômio (a saber o polinômio minimal de $A_{v}$ ), são funções contínuas, mas nem sempre são diferencíaveis. Por exemplo se $S$ é uma superfície de $\mathbf{E}^{3}, K$ e $H$ são suas curvaturas Gaussiana e média, respectivamente, então as curvaturas $\lambda$ de $S$ estão dadas por

$$
\lambda=H_{-}^{+} \sqrt{H^{2}-K},
$$

logo $\lambda$ não é diferencíavel no conjunto dos pontos umbílicos de $S$ (dizemos que $p \in M$ é um ponto umbilico de $M$ se as curvaturas principais nesse ponto coincidem). Porém, se as multiplicidades das curvaturas principais são constantes sobre o fibrado normal unitário, as curvaturas principais são funções suaves.

Seja $\xi$ um campo normal unitário local de $M$ definido em um subconjunto aberto e conexo $\mathcal{A}$ de $M$. Então $A_{\xi}$ é suavemente diagonalizável sobre um subconjunto aberto e denso $\mathcal{B}$ de $\mathcal{A}$. Sobre cada componente conexa $\mathcal{C}$ de $\mathcal{B}$ temos $g$ curvaturas principais $\lambda_{1}, \ldots, \lambda_{g}$, com multiplicidades $m_{1}, \ldots, m_{g}$. Os espaços de curvatura principal associados a cada $\lambda_{i}, i=1, \ldots, g$, definem $g$ distribuições $E_{1}, \ldots, E_{g}$, chamadas distribuições de curvatura. Para cada $p \in M$ o subespaço linear $E_{0}(p)=\bigcap_{v \in \nu_{p} M} \operatorname{ker} A_{v}$ de $T_{p} M$ é chamado espaço de nulidade de $M$ em $p$. A coleção dos espaços de nulidade de pontos de $\mathcal{C}$ constituem a distribuição de nulidade. Se $\xi$ está definido globalmente e as curvaturas principais são constantes ao longo de $\xi$ então as distribuições de curvatura estão definidas 
sobre $M$. Se alguma curvatura principal ao longo de $\xi$ é nula, a distribuição de curvatura associada coincide com a distribuição de nulidade. Para uma apresentação detalhada destes fatos vide [Rec79] e [Nom73].

Uma subvariedade conexa $S$ de $M$ diz-se superfície de curvatura se existe um campo normal paralelo $\xi$ tal que $T_{x} S$ está contido em um espaço de curvatura principal de $A_{\xi_{x}}$ para todo $x \in S$.

\subsection{Subvariedades totalmente geodésicas e subvariedades totalmen- te umbílicas}

\subsubsection{Subvariedades totalmente geodésicas}

Uma subvariedade $M$ de uma variedade Riemanniana $\bar{M}$ diz-se totalmente geodésica se toda geodésica de $M$ é uma geodésica de $\bar{M}$. Pela fórmula de Gauss $M$ é totalmente geodésica se e somente se a segunda forma fundamental de $M$ é nula.

Uma distribuição $\mathfrak{D}$ sobre uma variedade Riemanniana $\bar{M}$ diz-se auto-paralela se para cada par de seções $X$ e $Y$ de $\mathfrak{D}$ tem-se que $\bar{\nabla}_{X} Y$ é uma seção de $\mathfrak{D}$. Pelo Teorema de Frobenius toda distribuição auto-paralela é integrável. Uma distribuição integrável $\mathfrak{D}$ de $\bar{M}$ é auto-paralela se e somente se suas folhas são subvariedades totalmente geodésicas de $\bar{M}$.

As subvariedades conexas, completas e totalmente geodésicas de $\mathbf{E}^{n}$ e $\mathbf{S}^{n}$ são precisamente os subespaços afins e as hiperesferas equatoriais, respectivamente.

\subsubsection{Subvariedades totalmente umbílicas}

Uma subvariedade $M$ de uma variedade Riemanniana $\bar{M}$ diz-se umbílica em $p \in M$ na direção de $v \in \nu_{p} M$ se $A_{v}$ é múltiplo da identidade de $T_{p} M$. Um campo $\xi \in \Gamma(\nu M)$ tal que $A_{\xi}$ é múltiplo da identidade diz-se campo normal umbílico e dizemos que $M$ é umbílica na direção de $\xi$. Se $M$ é umbílica em toda direção normal dizemos que $M$ é uma subvariedade totalmente umbílica de $\bar{M}$.

O campo de curvatura média $H$ de $M$ é dado por

$$
H=\frac{1}{m} \operatorname{trac} o(\alpha) .
$$


$h=|H|$ é a função de curvatura média de $M$. Dizemos que $M$ é mínima se $h=0$.

Suponhamos que $M$ é totalmente umbílica. Dado $p \in M$, seja $e_{1}, \ldots, e_{m}$ uma base ortogonal para $T_{p} M$. Se $u, w \in T_{p} M, v \in \nu_{p} M$ e $\lambda$ é a curvatura principal de $A_{v}$. Por (1.2) temos que

$$
\begin{aligned}
\left\langle\alpha(u, w)-\langle u, w\rangle H_{p}, v\right\rangle & =\left\langle A_{v} u, w\right\rangle-\langle u, w\rangle\left\langle H_{p}, v\right\rangle \\
& =\langle u, w\rangle\left(\lambda-\left\langle H_{p}, v\right\rangle\right) \\
& =\langle u, w\rangle\left(\lambda-\frac{1}{m} \sum_{i}\left\langle\alpha\left(e_{i}, e_{i}\right), v\right\rangle\right) \\
& =\langle u, w\rangle\left(\lambda-\frac{1}{m} \sum_{i}\left\langle A_{v} e_{i}, e_{i}\right\rangle\right) \\
& =\langle u, w\rangle\left(\lambda-\frac{1}{m} \sum_{i} \lambda\left\langle e_{i}, e_{i}\right\rangle\right)=0 .
\end{aligned}
$$

Reciprocamente, se $\alpha=\langle\cdot, \cdot\rangle H$, então usando de novo (1.2), para $X, Y \in \Gamma(T M)$ e $\xi \in \Gamma(\nu M)$

$$
\left\langle A_{\xi} X, Y\right\rangle=\langle\alpha(X, Y), \xi\rangle=\langle\langle H, \xi\rangle X, Y\rangle
$$

logo $M$ é totalmente umbílica. Portanto $M$ é umbílica se e só se $\alpha=\langle\cdot, \cdot\rangle H$.

As subvariedades conexas, completas e totalmente umbílicas de $\mathbf{E}^{n}$ são os espaços afins e as intersecções de espaços afins com hiperesferas. As subvariedades conexas, completas e totalmente umbílicas de $\mathbf{S}^{n}$ são as intersecções de $\mathbf{S}^{n}$ com subespaços afins de $\mathbf{E}^{n+1}$.

\subsection{Redução de codimensão}

Dizemos que uma subvariedade $M$ de uma variedade Riemanniana $\bar{M}$ é substancial ${ }^{*}$ se $M$ não está contida em uma subvariedade totalmente geodésica de $\bar{M}$ de dimensão menor do que $n$.

$M$ é substancial em $\mathbf{E}^{n}$ se e somente se não existe um hiperplano afim de $\mathbf{E}^{n}$ contendo $M$. Se $M$ não é substancial em $\mathbf{E}^{n}$ seja $F$ a intersecção dos hiperplanos afins que o contem. $F$ é o menor subespaço afim de $\mathbf{E}^{n}$ que contem $M$. Assim poderemos considerar $M$ como uma subvariedade de $\mathbf{E}^{l}$ onde $l=\operatorname{dim} F$.

\footnotetext{
*Do inglês 'substancial'.
} 
Dado $p \in M$ seja $\mathcal{N}_{p}$ o subespaço linear de $\nu_{p} M$ gerado por $\left\{\alpha(u, w): u, w \in T_{p} M\right\}$. Dizemos que $\mathcal{N}_{p}$ é o primeiro espaço normal de $M$ em $p$. Tem-se que $\mathcal{N}_{p}$ é o complemento ortogonal de $\left\{v \in \nu_{p} M: A_{v}=0\right\}$ em $\nu_{p} M$. Se a dimensão de $\mathcal{N}_{p}$ independe de $p \in M$, então $\mathcal{N}=\left\{\mathcal{N}_{p}: p \in M\right\}$ é um subfibrado vetorial de $\nu M$.

Teorema 1.5.1 (Redução de codimensão). Seja $M$ uma subvariedade conexa de uma forma espacial $\bar{M}$. Suponhamos que $\mathcal{N}$ é invariante pelo transporte paralelo de $\nu M$. Então existe uma subvariedade totalmente geodésica $N$ de $\bar{M}$, contendo $M$, com $\operatorname{dim} N=l+m$, onde l é a dimensão das fibras de $\mathcal{N}$.

Demonstração. Sem perder generalidade podemos supor que a curvatura seccional $\kappa$ de $\bar{M}$ é 0,1 ou -1 . Só faremos a prova para o caso $\kappa=0,1$; a prova do caso $\kappa=-1$ é similar.

Seja $p \in M$ fixo. Dado $q \in M$, como $M$ é conexa, existe uma curva suave por partes $\gamma:[0,1] \longrightarrow$ $M \operatorname{com} \gamma(0)=p$ e $\gamma(1)=q$. Sejam $v \in \mathcal{N}^{\perp}$ e $\xi$ o transporte paralelo de $v$ ao longo de $\gamma$. Temos que $\nu M=\mathcal{N} \oplus \mathcal{N}^{\perp}$. Então $\mathcal{N}^{\perp}$ também é invariante pelo transporte paralelo de $\nu M$. Assim

$$
\bar{\nabla}_{\gamma^{\prime}(t)} \xi(t)=-A_{\xi(t)} \gamma^{\prime}(t)+\nabla_{\gamma^{\prime}(t)}^{\perp} \xi(t)=0
$$

para todo $t \in[0,1]$.

a. Suponhamos que $\bar{M}=\mathbf{E}^{n}$. Então

$$
\xi^{\prime}(t)=\bar{\nabla}_{\gamma^{\prime}(t)} \xi(t)
$$

para todo $t \in[0,1]$. Segue que $\xi$ é constante, $\log \langle\gamma(t), \xi(t)\rangle=\langle p, v\rangle$, para todo $t \in[0,1]$, em particular $\langle q-p, v\rangle=0$. Portanto, se $v_{1}, \ldots, v_{n-m-l}$ é uma base ortogonal de $\mathcal{N}_{p}^{\perp}$, tem-se $\left\langle q-p, v_{i}\right\rangle=0$ para todo $q \in M$ e todo $i=1, \ldots, n-m-l$. Fazendo $N=p+\cap_{i=1}^{n-m-l} v_{i}^{\perp}$ obtemos

$$
M \subset N
$$

$\operatorname{com} \operatorname{dim} N=m+l$.

b. Suponhamos que $\bar{M}=\mathbf{S}^{n}$. Temos que

$$
\xi^{\prime}(t)-\left\langle\xi^{\prime}(t), \gamma(t)\right\rangle \gamma(t)=\bar{\nabla}_{\gamma^{\prime}(t)} \xi(t)
$$


para todo $t \in[0,1]$. De outro lado $\langle\xi(t), \gamma(t)\rangle=0$, logo

$$
0=\frac{d}{d t}\langle\xi(t), \gamma(t)\rangle=\left\langle\xi^{\prime}(t), \gamma(t)\right\rangle+\left\langle\xi(t), \gamma^{\prime}(t)\right\rangle=\left\langle\xi^{\prime}(t), \gamma(t)\right\rangle
$$

então $\xi(t)=v$ para todo $t \in[0,1]$, em particular $\langle q, v\rangle=0$. Portanto, se $v_{1}, \ldots, v_{n-m-l}$ é uma base ortogonal de $\mathcal{N}_{p}^{\perp}$, tem-se $\left\langle q, v_{i}\right\rangle=0$ para todo $q \in M$ e todo $i=1, \ldots, n-m-l$. Fazendo $N=\mathbf{S}^{n} \cap\left(\cap_{i=1}^{n-m-l} v_{i}^{\perp}\right)$ obtemos

$$
M \subset N \subset \mathbf{S}^{n},
$$

$\operatorname{com} \operatorname{dim} N=m+l$.

\subsection{Submersões Riemannianas}

Sejam $M$ e $N$ duas variedades Riemannianas. Una aplicação sobrejetora e suave $\pi: M \longrightarrow N$ diz-se submersão Riemanniana se

$$
\left.d \pi_{p}\right|_{\left(\operatorname{ker} d \pi_{p}\right)^{\perp}}:\left(\operatorname{ker} d \pi_{p}\right)^{\perp} \longrightarrow T_{\pi(p)} N
$$

é uma isometria linear para todo $p \in M$. As distribuições $\mathcal{V}$ e $\mathcal{H}$ sobre $M$ dadas por $\mathcal{V}_{p}=\operatorname{ker} d \pi_{p}$ e $\mathcal{H}_{p}=\left(\mathcal{V}_{p}\right)^{\perp}$, para todo $p \in M$, são chamadas distribuições vertical e horizontal de $M$ em relação a $\pi$, respectivamente. As componentes conexas das fibras de $\pi$ são variedades integrais de $\mathcal{V}$. Temos a decomposição ortogonal $T M=\mathcal{V} \oplus \mathcal{H} . \mathcal{H}$ nem sempre é integrável.

Uma curva $\gamma: I \longrightarrow M$ diz-se levantamento horizontal da curva $\beta: I \longrightarrow N$, se $\beta=\pi \circ \gamma$ e $\gamma$ é horizontal, isto é $\gamma^{\prime}(t) \in \mathcal{H}_{\gamma(t)}$, para todo $t \in I$. Dada $\beta: I \longrightarrow N$, se $t_{o} \in I$ e $p_{0} \in \pi^{-1}\left(\beta\left(t_{0}\right)\right)$, existe um único levantamento horizontal $\gamma: I_{0} \subset I \longrightarrow M$ de $\left.\beta\right|_{I_{o}}$ passando por $p_{0}$, ou seja, com $t_{0} \in I_{0}$ e $\gamma\left(t_{0}\right)=p_{0}$. Se $\gamma$ é uma curva em $M$ então $|\gamma| \geq|d(\pi \circ \gamma)|$ e tem-se a igualdade se e somente se $\gamma$ é horizontal, isto segue da equação (1.9). Portanto o comprimento de $\gamma$ é maior ou igual ao comprimento de $d(\pi \circ \gamma)$ e dá-se a igualdade se e somente se $\gamma$ é horizontal. Assim o levantamento horizontal de uma geodésica é uma geodésica. Em particular se $\mathcal{H}$ é integrável então suas folhas são totalmente geodésicas.

Um campo $X \in \Gamma(T M)$ diz-se projetável se existe um campo $Z \in \Gamma(T N) \pi$-relacionado com $X$, 
isto é $d \pi_{p} X_{p}=Z_{\pi(p)}$, para todo $p \in M$. Logo $X$ é projetável se e somente se $d \pi(X)$ é constante ao longo das fibras de $\pi$. Dado um campo $Z \in \Gamma(T N)$ existe um único campo horizontal $X \in \Gamma(\mathcal{H})$ que é $\pi$-relacionado com $Z$. Dizemos que $X$ é o levantamento horizontal de $Y$. Um campo projetável e horizontal diz-se campo vetorial básico. Note que se $U$ é um campo vertical, isto é $U \in \Gamma(\mathcal{V})$, então $U$ é $\pi$-relacionado com o campo nulo, ademais sabemos que para dois pares de campos $\pi$-relacionados seus respectivos colchetes são $\pi$-relacionados. Assim o colchete de dois campos projetáveis é projetável e o colchete de um campo vertical por um campo projetável é vertical.

Sejam $U \in \Gamma(\mathcal{V})$ e $X, Y \in \Gamma(\mathcal{H})$ dois campos básicos. Então

$$
\langle[U, X], Y\rangle=0=\langle[U, Y], X\rangle .
$$

Como $X$ e $Y$ são constantes ao longo fibras de $\pi$, temos que $U\langle X, Y\rangle=0$. Logo da fórmula de Koszul obtemos

$$
\left\langle\nabla_{X} Y, U\right\rangle=\frac{1}{2}\langle[X, Y], U\rangle .
$$

Proposição 1.6.1. Seja $\pi: M \longrightarrow N$ uma submersão Riemanniana. Então as seguintes afirmações são equivalentes

a. A distribuição horizontal é integrável

b. A distribuição horizontal é totalmente geodésica

c. Sobre cada fibra de $\pi$ os campos básicos são normais paralelos.

Demonstração. Suponhamos que $\mathcal{H}$ é integrável. Pelo teorema de Frobenius $\mathcal{H}$ é involutiva, então o membro direito da equação (1.11) anula-se. Segue que $\mathcal{H}$ é auto paralela e portanto tem folhas totalmente geodésicas.

Suponhamos que a distribuição horizontal é totalmente geodésica. Assim o lado esquerdo da equação (1.11) anula-se. Os campos básicos são seções normais das fibras de $\pi$, combinando os membros esquerdos das equações (1.10) e (1.11) segue que estas são paralelas.

Suponhamos que sobre cada fibra de $\pi$ os campos básicos são paralelos. Assim o membro direito da equação (1.11) anula-se. Segue que a distribuição horizontal e involutiva então, pelo teorema de Frobenius, $\mathcal{H}$ é integrável. 


\section{CAPÍTULO 2}

\section{Subvariedades isoparamétricas}

Como ficou esclarecido na introdução deste trabalho, o conceito de subvariedade isoparamétrica nasce junto com o de aplicação isoparamétrica dado por Harle. Mas estes dois são bem posteriores aos trabalhos feitos sobre funções isopárametricas e famílias de hipersuperfícies isoparamétricas. Neste capítulo damos a definição no sentido de Terng e desenvolvemos varias propriedades básicas destes objetos. Deixamos para a última seção a discussão sobre aplicações isoparamétricas.

Seja $M$ uma subvariedade de uma forma espacial $\bar{M}$. Dizemos que $M$ tem curvaturas principais constantes se para cada campo normal paralelo ao longo de uma curva suave por partes, as curvaturas principais na direção desse campo são constantes. Se ainda $M$ tem fibrado normal raso dizemos que $M$ é uma subvariedade isoparamétrica de $\bar{M}$.

Proposição 2.1. Seja $M$ uma subvariedade de $\mathbf{S}^{n} \subset \mathbf{E}^{n+1}$. Então $M$ é isoparamétrica vista como subvariedade de $\mathbf{S}^{n}$ se e somente se é isoparamétrica vista como subvariedade de $\mathbf{E}^{n+1}$.

Demonstração. Denotemos com $A$ e $\hat{A}$ os operadores de forma, com $\nabla^{\perp}$ e $\nabla^{\hat{\perp}}$ as conexões normais e com $\nu M$ e $\hat{\nu} M$ os fibrados normais de $M$, vista como subvariedade de $\mathbf{E}^{n+1}$ e $\mathbf{S}^{n}$, respectivamente. Seja $P$ é o campo vetor posição de $\mathbf{E}^{n+1}$ restrito a $\mathbf{S}^{n}$. Seja $\zeta \in \Gamma(\nu M)$, então $\zeta=-f P+\xi$, onde 
$\xi \in \Gamma(\hat{\nu} M)$ e $f: M \longrightarrow \mathbf{R}$ é una função suave. Como $\langle X, \xi\rangle=0$, temos que

$$
\langle(d \xi) X, P\rangle P=(X\langle\xi, P\rangle-\langle\xi, X\rangle) P=0
$$

logo da fórmula de Weingarten

$$
\begin{aligned}
-A_{\zeta} X+\nabla_{X}^{\perp} \zeta & =d \zeta(X) \\
& =d \xi(X)-X(f) P-f X \\
& =d \xi(P)-\langle d \xi(X), P\rangle P-X(f) P-f X \\
& =-\hat{A}_{\xi} X+\nabla \frac{\hat{\perp}}{X} \xi-X(f) P-f X .
\end{aligned}
$$

Portanto

$$
A_{\zeta} X=\hat{A}_{\xi} X+f X
$$

e

$$
\nabla_{X}^{\perp} \zeta=\nabla_{X}^{\hat{\Lambda}} \xi-X(f) P
$$

Por (2.2) $\zeta$ é paralelo em relação a $\nabla^{\perp}$ se e somente se $\xi$ é paralelo em relação a $\nabla^{\hat{\perp}}$ e $f$ e constante. Neste caso, a equação (2.1) implica que os auto-valores de $A_{\zeta}$ são da forma $\hat{\lambda}+\mu$ onde $\hat{\lambda}$ é um auto-valor de $\hat{A}_{\xi}$ e $\mu$ é um número real. Então $M$ tem curvaturas principais constantes em relação a $\mathbf{S}^{n}$ se e somente se tem curvaturas principais constantes em relação a $\mathbf{E}^{n+1}$

Finalmente se $Y \in \Gamma(T M)$, da equação (2.2) temos que

$$
\begin{aligned}
\left(\nabla_{X}^{\perp} \nabla_{Y}^{\perp}-\nabla_{Y}^{\perp} \nabla_{X}^{\perp}-\nabla_{[X, Y]}^{\perp}\right) \zeta & =\left(\nabla_{X}^{\hat{\imath}} \nabla_{Y}^{\hat{\imath}}-\nabla_{Y}^{\hat{\imath}} \nabla_{X}^{\hat{\imath}}-\nabla_{[X, Y]}^{\hat{\imath}}\right) \xi+ \\
& -(X Y-Y X-[X, Y])(f) P \\
& =\left(\nabla_{X}^{\hat{\imath}} \nabla_{Y}^{\hat{\imath}}-\nabla_{Y}^{\hat{\imath}} \nabla_{X}^{\hat{\imath}}-\nabla_{[X, Y]}^{\hat{\imath}}\right) \xi
\end{aligned}
$$

então $\nu M$ é raso se e somente se $\hat{\nu} M$ é raso.

Neste capítulo estudaremos a geometria das subvariedades isoparamétricas do espaço Euclidiano. Pela proposição acima, na nossa discussão estarão incluídas as subvariedades isoparamétricas da esfera. 


\subsection{Variedades focais e paralelas}

Seja $M$ uma subvariedade de $\mathbf{E}^{n}$. Suponhamos que existe um campo normal paralelo não trivial $\xi$ definido em $M$. Consideremos a aplicação $\pi: M \longrightarrow \mathbf{E}^{n}$ dada por $\pi(p)=p+\xi(p)$. Temos que $d \pi_{p}=i d_{T_{p} M}-A_{\xi_{p}}$, para cada $p \in M$. Seja $v(p)=\operatorname{dim}\left(\operatorname{ker}\left(d \pi_{p}\right)\right), p \in M$. Um ponto $\pi(p)$ diz-se ponto focal na direção de $\xi$ se $v(p)>0$. Se $v$ é constante $M_{\xi}=\pi(M)$ é uma subvariedade imersa de $\mathbf{E}^{n}$, com $\operatorname{dim} M_{\xi}=m-v$. Se $v>0$ é constante dizemos que $M_{\xi}$ é uma variedade focal de $M$. Se $v=0, M_{\xi}$ diz-se variedade paralela de $M$.

Nesta seção $\hat{\nabla}$ e $\hat{A}$ denotarão a conexão de Levi-Civia e o operador de forma de $M_{\xi}$, respectivamente.

Observação 2.1.1. Na verdade $M_{\xi}$ é focal se e somente se $A_{\xi}$ tem um auto-valor constante $\lambda=1$, ao longo do campo paralelo $\xi$. Com efeito, se $p \in M$, então $v(p)>0$ se e só se $\operatorname{ker}\left(d \pi_{p}\right)$ é o auto-espaço associado ao auto-valor 1 de $A_{\xi_{p}}$.

Seja $M_{\xi}$ uma variedade focal de $\mathrm{M}$, claramente $\pi: M \longrightarrow M_{\xi}$ é uma submersão. Sejam $\mathcal{V}$ e $\mathcal{H}$, as distribuições definidas sobre $M$ onde $\mathcal{V}_{p}=\operatorname{ker}\left(d \pi_{p}\right)$ e $\mathcal{H}_{p}=\mathcal{V}_{p}^{\perp}$ é o complemento ortogonal de $\mathcal{V}_{p}$ em relação a $T_{p} M$, para todo $p \in M$. Dado $p \in M$, se $u \in \mathcal{V}_{p}$ e $w \in T_{p} M$, como $A_{\xi_{p}}$ é auto-adjunto

$$
\left\langle d \pi_{p} w, u\right\rangle=\left\langle w-A_{\xi_{p}} w, u\right\rangle=\langle w, u\rangle-\left\langle w, A_{\xi_{p}} u\right\rangle=0 .
$$

Logo $T_{\pi(p)} M_{\xi} \subset \mathcal{H}_{p}$ e $\operatorname{dim}\left(T_{\pi(p)} M_{\xi}\right)=\operatorname{dim}\left(\mathcal{H}_{p}\right)$, portanto $T_{\pi(p)} M_{\xi}=\mathcal{H}_{p}$ e $\nu_{\pi(p)} M_{\xi}=\mathcal{V}_{p} \oplus \nu_{p} M$. Em particular se $M_{\xi}$ é paralela tem-se $T_{\pi(p)} M_{\xi}=T_{p} M$ e $\nu_{\pi(p)} M_{\xi}=\nu_{p} M$, para todo $p \in M$.

Lema 2.1.2. Seja $M_{\xi}$ uma variedade focal de $M \subset \mathbf{E}^{n}$. Consideremos a projeção $\pi: M \longrightarrow M_{\xi}$. Então as componentes conexas das fibras de $\pi$ são subvariedades totalmente geodésicas de $M$ e os operadores de forma de $M$ preservam a decomposição ortogonal $T M=\mathcal{V} \oplus \mathcal{H}$.

Demonstração. Sejam $U$ e $V$ seções de $\mathcal{V}$. Então se $X$ uma seção de $\mathcal{H}$, como $\xi$ é paralelo, da equação 
de Codazzi temos que

$$
\begin{aligned}
\left\langle\nabla_{U} V, X\right\rangle & =\left\langle\nabla_{U}\left(A_{\xi} V\right), X\right\rangle \\
& =\left\langle\left(\nabla_{U} A\right)_{\xi} V, X\right\rangle+\left\langle A_{\xi}\left(\nabla_{U} V\right), X\right\rangle \\
& =\left\langle\left(\nabla_{X} A\right)_{\xi} V, U\right\rangle+\left\langle\nabla_{U} V, A_{\xi} X\right\rangle \\
& =\left\langle\nabla_{X}\left(A_{\xi} V\right), U\right\rangle-\left\langle A_{\xi}\left(\nabla_{X} V\right), U\right\rangle+\left\langle\nabla_{U} V, A_{\xi} X\right\rangle \\
& =\left\langle\nabla_{X} V, U-A_{\xi} U\right\rangle+\left\langle\nabla_{U} V, A_{\xi} X\right\rangle \\
& =\left\langle\nabla_{U} V, A_{\xi} X\right\rangle .
\end{aligned}
$$

Então $\left\langle\nabla_{U} V, d \pi(X)\right\rangle=\left\langle\nabla_{U} V, X-A_{\xi} X\right\rangle=0$, para toda seção $X$ de $\mathcal{H}$. Logo $\nabla_{U} V \in \Gamma(\mathcal{V})$ para todo par de seções $U, V \in \Gamma(\mathcal{V})$. Ou seja $\mathcal{V}$ é uma distribuição auto-paralela, assim suas folhas (que são precisamente as componentes conexas das fibras de $\pi$ ) são subvariedades totalmente geodésicas de $M$.

De outro lado, como $\xi$ é paralelo a equação de Ricci implica que $A_{\eta}$ comuta com $A_{\xi}$, para todo $\eta \in \Gamma(\nu M)$. Logo os operadores de forma de $M$ preservam $\mathcal{V}$ e por serem auto-adjuntos preservam $\mathcal{H}$.

Observação 2.1.3. Seja $\mathfrak{D}$ uma distribuição suave sobre $M$. Então as seguintes afirmações são equivalentes

a. Os operadores de forma de $M$ preservam $\mathfrak{D}$.

b. $\alpha(u, w)=0$, para todo $u \in \mathfrak{D}_{p}, w \in\left(\mathfrak{D}_{p}\right)^{\perp}$ e todo $p \in M$.

c. Os operadores de forma de $M$ preservam $\mathfrak{D}^{\perp}$.

Com efeito, seja $v \in \nu_{p} M$, então

$$
\langle\alpha(u, w), v\rangle=\left\langle A_{v} u, w\right\rangle=\left\langle u, A_{v} w\right\rangle
$$

Seja $\beta:(-\epsilon, \epsilon) \longrightarrow M_{\xi}$ uma curva suave com $\beta(0)=\hat{p}$. Dado $p \in \pi^{1-}(\hat{p})$, seja $\gamma:(-\epsilon, \epsilon) \longrightarrow M$ o levantamento horizontal de $\beta$ passando por $p$. Se $\zeta$ é um campo normal ao longo de $\beta$ com $\zeta(t) \in \nu_{\gamma(t)} M \subset \nu_{\beta(t)} M_{\xi}$, para todo $t \in(-\epsilon, \epsilon)$. Então o campo $\eta(t)=\zeta(t)$, para todo $t \in(-\epsilon, \epsilon)$, 
define um campo normal $\eta$ ao longo de $\gamma$. Reciprocamente, se $\eta$ é um campo normal ao longo de $\gamma$, então $\zeta(t)=\eta(t)$, define um campo normal $\zeta$ ao longo de $\beta \operatorname{com} \zeta(t) \in \nu_{\gamma(t)} M \subset \nu_{\beta(t)} M_{\xi}$, para todo $t \in(-\epsilon, \epsilon)$.

Lema 2.1.4. Com a notação acima temos que

$$
\hat{A}_{\zeta(t)} \beta^{\prime}(t)=A_{\eta(t)} \gamma^{\prime}(t)
$$

$e$

$$
\left(\hat{\nabla} \frac{\perp}{\beta^{\prime}} \zeta\right)(t)=\left(\nabla \frac{\perp}{\gamma^{\prime}} \eta\right)(t)
$$

para todo $t \in(-\epsilon, \epsilon)$. Em particular o transporte paralelo, de vetores de $\nu_{p} M$, ao longo de $\gamma$ e ao longo de $\beta$ coincidem.

Demonstração. Pela equação de Weingarten temos que

$$
-\hat{A}_{\zeta(t)} \beta^{\prime}(t)+\left(\hat{\nabla}_{\beta^{\prime}}^{\perp} \zeta\right)(t)=\zeta^{\prime}(t)=\eta^{\prime}(t)=-A_{\eta(t)} \gamma^{\prime}(t)+\left(\nabla_{\gamma^{\prime}}^{\perp} \eta\right)(t)
$$

para todo $t \in(-\epsilon, \epsilon)$. Do lema (2.1.2) sabemos que os operadores de forma de $M$ deixam $\mathcal{H}$ invariante. Assim como $\gamma$ é o levantamento horizontal de $\beta$, então $-A_{\eta(t)} \gamma^{\prime}(t) \in \mathcal{H}_{\gamma(t)}=T_{\beta(t)} M_{\xi}$, para todo $t \in(-\epsilon, \epsilon)$. Daqui seguem as equações $(2.3)$ e $(2.4)$.

Corolário 2.1.5 ("Fórmula do tubo"). Suponhamos que $M_{\xi}$ é uma variedade paralela ou focal de uma subvariedade $M$ do espaço Euclidiano. Então se $p \in M$ e $v \in \nu_{p} M$, temos que

$$
\hat{A}_{v}=A_{v}\left(\left.\left(i d_{T_{p} M}-A_{\xi_{p}}\right)\right|_{\mathcal{H}_{p}}\right)^{-1} .
$$

Demonstração. Com a notação do lema acima, sejam $v=\eta(0)=\zeta(0)$ e $w=\beta^{\prime}(0)=$, então a equação (2.3) e o fato de que $\gamma$ é o levantamento horizontal de $\beta$ implicam que

$$
\hat{A}_{v} w=A_{v} \gamma^{\prime}(0)=A_{v}\left(\left.\left(i d_{T_{p} M}-A_{\xi_{p}}\right)\right|_{\mathcal{H}_{p}}\right)^{-1} w .
$$

Seja $M$ uma subvariedade do espaço Euclidiano, com fibrado normal raso. Para cada $p \in M$ denotamos com $F_{M}(p)$ o conjunto focal de $M$ em $p$, constituído pelos pontos em $\mathbf{E}^{n}$ da forma $p+v$, 
onde $v \in \nu_{p} M$ tal que $A_{v}$ tem um autovalor igual a 1 .

Proposição 2.1.6. Seja $M \subset \mathbf{E}^{n}$ uma subvariedade com fibrado normal raso. Suponhamos que existe uma campo normal paralelo $\xi \in \Gamma(\nu M)$ tal que $M_{\xi}$ é uma variedade paralela de $M$. Consideremos a projeção $\pi: M \longrightarrow M_{\xi}$, então para todo $p \in M$ tem-se

a. $p+\nu_{p} M=\pi(p)+\nu_{\pi(p)} M_{\xi}$

b. $F_{M}(p)=F_{M_{\xi}}(\pi(p))$

Demonstração. a. Temos que $\xi_{p} \in \nu_{p} M$ e $\nu_{p} M=\nu_{\pi(p)} M_{\xi}$. Então

$$
p+\nu_{p} M=p+\xi_{p}+\nu_{p} M=\pi(p)+\nu_{p} M=\pi(p)+\nu_{\pi(p)} M_{\xi} .
$$

b. Seja $v \in \nu_{p} M$, da fórmula do tubo temos que

$$
\begin{aligned}
\hat{A}_{v-\xi_{p}} & =\hat{A}_{v}-\hat{A}_{\xi_{p}} \\
& =A_{v}\left(i d_{T_{p} M}-A_{\xi_{p}}\right)^{-1}-A_{\xi_{P}}\left(i d_{T_{p} M}-A_{\xi_{p}}\right)^{-1} \\
& =\left(A_{v}-A_{\xi_{p}}\right)\left(i d_{T_{p} M}-A_{\xi_{p}}\right)^{-1} \\
& =\left(i d_{T_{p} M}-A_{\xi_{P}}-\left(i d_{T_{p} M}-A_{v}\right)\right)\left(i d_{T_{p} M}-A_{\xi_{p}}\right)^{-1} \\
& =i d_{T_{p} M}-\left(i d_{T_{p} M}-A_{v}\right)\left(i d_{T_{p} M}-A_{\xi_{p}}\right)^{-1} \\
& =i d_{T_{\pi(p)} M_{\xi}}-\left(i d_{T_{p} M}-A_{v}\right)\left(i d_{T_{p} M}-A_{\xi_{p}}\right)^{-1} .
\end{aligned}
$$

Então $i d_{T_{\pi(p)} M_{\xi}}-\hat{A}_{v-\xi_{p}}$ é singular se e só se $i d_{T_{p} M}-A_{v}$ é singular, logo

$$
\begin{aligned}
F_{M}(p) & =\left\{p+v: i d_{T_{p} M}-A_{v} \text { é singular }\right\} \\
& =\left\{\pi(p)+\left(v-\xi_{p}\right): i d_{T_{\pi(p)} M_{\xi}}-\hat{A}_{v-\xi_{p}} \text { é singular }\right\} \\
& =F_{M_{\xi}}(\pi(p)) .
\end{aligned}
$$




\subsection{Normais de curvatura}

Seja $M$ uma subvariedade isoparamétrica do espaço Euclidiano. Como o tensor de curvatura do fibrado normal de $M$ é nulo, a equação de Ricci implica que para todo $p \in M,\left\{A_{v}: v \in \nu_{p} M\right\}$ é uma família comutativa de operadores auto-adjuntos de $T_{p} M$. Logo são simultaneamente diagonalizáveis com auto-valores $\lambda_{1}(p), \ldots, \lambda_{g}(p) \in\left(\nu_{p} M\right)^{*}$ e auto-espaços comuns $E_{1}(p), \ldots, E_{g}(p)$. Como as curvaturas principais são constantes ao longo de campos paralelos obtemos $g$ distribuições de curvatura $E_{1}, \ldots, E_{g}$, mutuamente ortogonais com $T M=\oplus_{i} E_{i}$. Como $\lambda_{1}, \ldots, \lambda_{g} \in(\nu M)^{*}$, existem $g$ campos normais $\mathbf{n}_{1}, \ldots, \mathbf{n}_{g}$, tais que $\lambda_{i}(p, v)=\lambda_{i}(v)=\left\langle\mathbf{n}_{i}(p), v\right\rangle$, para todo $i \in\{1, \ldots, g\}$, todo $v \in \nu_{p} M$ e todo $p \in M$. Estes campos são chamados normais de curvatura. Quando exista uma normal de curvatura nula para distinguir-la das outras será denotada por $\mathbf{n}_{0}$.

As normais de curvatura são campos paralelos. Com efeito, dado $i \in\{1, \ldots, g\}$ seja $\xi$ um campo normal paralelo. Sabemos que $\lambda_{i}(\xi)$ é constante, então para todo $X \in \Gamma(T M)$ tem-se

$$
\left\langle\nabla_{X}^{\perp} \mathbf{n}_{i}, \xi\right\rangle=X\left\langle\mathbf{n}_{i}, \xi\right\rangle-\left\langle\mathbf{n}_{i}, \nabla_{X}^{\perp} \xi\right\rangle=X\left(\lambda_{i}(\xi)\right)=0 .
$$

Observação 2.2.1. Se $M \subset \mathbf{E}^{n}$ é uma subvariedade isoparamétrica então o primeiro espaço normal é gerado pelas normais de curvatura. Com efeito, dados $p \in M$ e $v \in \nu_{p} M$ então $v$ é ortogonal ao primeiro espaço normal de $M$ em $p$ se e somente se o operador de forma na direção de $v$ e nulo, se e somente se $v$ e ortogonal a todas as normais de curvatura em $p$, se e somente se $v$ pertence ao espaço ortogonal ao espaço gerado pelas normais de curvatura em $p$.

Proposição 2.2.2. Seja $M_{\xi}$ uma variedade paralela de uma subvariedade isoparamétrica $M$ do espaço Euclidiano. Então $M_{\xi}$ é isoparamétrica.

Demonstração. Consideremos a projeção $\pi: M \longrightarrow M_{\xi}$. Dados campos $X, Y \in \Gamma(T M)$ e $\eta \in \Gamma(\nu M)$, $\operatorname{sejam} Z, W \in \Gamma\left(T M_{\xi}\right)$ e $\zeta \in \Gamma\left(\nu M_{\xi}\right), \operatorname{com} Z_{\pi(p)}=d \pi_{p} X_{p}, W_{\pi(p)}=d \pi_{p} Y_{p}$ e $\zeta_{\pi(p)}=\eta_{p}$, para todo $p \in M$. Note que as seções tangentes e normais de $M_{\xi}$ são desta forma. Pelo lema (2.1.4) temos que

$$
\left(\hat{\nabla}_{Z}^{\perp} \zeta\right)_{\pi(p)}=\left(\nabla \frac{\perp}{X} \eta\right)_{p}
$$


Assim, denotando com $\hat{R}$ o tensor de curvatura normal de $M_{\xi}$, tem-se

$$
(\hat{R}(Z, W) \zeta)_{\pi(p)}=(R(X, Y) \eta)_{p}
$$

para todo $p \in M$. Logo $M_{\xi}$ tem fibrado normal raso se $M$ tem fibrado normal raso.

Seja $p \in M$, fixo. Sabemos que $T_{p} M=T_{\pi(p)} M_{\xi}$ e $\nu_{p} M=\nu_{\pi(p)} M_{\xi} . \quad\left(i d_{T_{p} M}-A_{\xi_{p}}\right)^{-1}$ é um operador auto-adjunto de $T_{p} M$ que comuta com $A_{v}$, para todo $v \in \nu_{p} M$. Então da fórmula do tubo a família $\left\{A_{v}, \hat{A}_{v}: v \in \nu_{p} M\right\}$ é simultaneamente diagonalizável. Portanto existe uma permutação $\sigma$ do conjunto $\{1, \ldots, g\}$ tal que $E_{j}(p)=E_{\sigma(j)}(\pi(p))=d \pi_{p} E_{j}(p)$. Seja $u \in E_{j}(p)$ então $u^{\prime}=d \pi_{p} u \in$ $E_{\sigma(j)}(\pi(p))$. Assim se $\hat{\lambda}_{1}, \ldots, \hat{\lambda}_{g}$ são as curvaturas principais de $M_{\xi}$, dado $v \in \nu_{p} M$ novamente pela fórmula do tubo tem-se

$$
\begin{aligned}
\lambda_{j}(v) u & =A_{v} u \\
& =A_{v}\left(d \pi_{p}\right)^{-1} u^{\prime} \\
& =A_{v}\left(i d_{T_{p} M}-A_{\xi_{p}}\right)^{-1} u^{\prime} \\
& =\hat{A}_{v} u^{\prime} \\
& =\hat{\lambda}_{\sigma(j)}(v) u^{\prime} \\
& =\hat{\lambda}_{\sigma(j)}(v) d \pi_{p} u \\
& =\hat{\lambda}_{\sigma(j)}(v)\left(i d_{T_{p} M}-A_{\xi_{p}}\right) u \\
& =\hat{\lambda}_{\sigma(j)}(v)\left(1-\lambda_{j}\left(\xi_{p}\right)\right) u .
\end{aligned}
$$

Da observação $(2.1 .1) \xi_{p} \notin F_{M}(p), \log 01-\lambda_{j}\left(\xi_{p}\right) \neq 0$ e portanto

$$
\hat{\lambda}_{\sigma(j)}(v)=\frac{\lambda_{j}(v)}{1-\lambda_{j}\left(\xi_{p}\right)}
$$

para todo $j \in\{1, \ldots, g\}$. Portanto $M_{\xi}$ tem curvaturas principais constantes ao longo de campos normais paralelos.

Lema 2.2.3. Dados $i, j, k \in\{1, \ldots, g\}$ e seções $X_{l}$ de $E_{l}, l=i, j, k$, tem-se

$$
\left(\nabla_{X_{i}}^{\perp} \alpha\right)\left(X_{j}, X_{k}\right)=\left\langle\nabla_{X_{i}} X_{j}, X_{k}\right\rangle\left(\mathbf{n}_{j}-\mathbf{n}_{k}\right) .
$$


Assim da equação de Codazzi obtemos

$$
\left\langle\nabla_{X_{i}} X_{j}, X_{k}\right\rangle\left(\mathbf{n}_{j}-\mathbf{n}_{k}\right)=\left\langle\nabla_{X_{j}} X_{i}, X_{k}\right\rangle\left(\mathbf{n}_{i}-\mathbf{n}_{k}\right)
$$

Demonstração. Seja $\xi$ um campo normal paralelo. $\lambda_{i}, \lambda_{j}$ e $\lambda_{k}$ são constantes ao longo de $\xi$. Então pela equação (1.3) temos que

$$
\begin{aligned}
\left(\lambda_{j}-\lambda_{k}\right)\left\langle\nabla_{X_{i}} X_{j}, X_{k}\right\rangle & =\left\langle\nabla_{X_{i}}\left(A_{\xi} X_{j}\right), X_{k}\right\rangle-\left\langle\nabla_{X_{i}} X_{j}, A_{\xi} X_{k}\right\rangle \\
& =\left\langle\nabla_{X_{i}}\left(A_{\xi} X_{j}\right), X_{k}\right\rangle-\left\langle A_{\xi}\left(\nabla_{X_{i}} X_{j}\right), X_{k}\right\rangle \\
& =\left\langle\left(\nabla_{X_{i}} A\right)_{\xi} X_{j}, X_{k}\right\rangle \\
& =\left\langle\left(\nabla_{X_{i}}^{\perp} \alpha\right)\left(X_{j}, X_{k}\right), \xi\right\rangle .
\end{aligned}
$$

Ou seja

$$
\left\langle\left\langle\nabla_{X_{i}} X_{j}, X_{k}\right\rangle\left(\mathbf{n}_{j}-\mathbf{n}_{k}\right)-\left(\nabla_{X_{i}}^{\perp} \alpha\right)\left(X_{j}, X_{k}\right), \xi\right\rangle=0,
$$

para todo campo normal paralelo $\xi$. Esta última equação é equivalente a (2.6).

Dado $p_{0} \in M$ consideremos um subespaço afim $\mathcal{P}$ de $\nu_{p_{0}} M$. Introduzimos a seguinte notação

$$
\begin{aligned}
& \mathfrak{D}_{\mathcal{P}}(p)=\bigoplus_{\mathbf{n}_{i}\left(p_{0}\right) \in \mathcal{P}} E_{i}(p), \\
& \nu_{\mathcal{P}}(p)=\sum_{\mathbf{n}_{i}\left(p_{0}\right) \in \mathcal{P}} \boldsymbol{R}_{i}(p), \\
& \mathbf{F}_{\mathcal{P}}(p)=\mathfrak{D}_{\mathcal{P}}(p) \oplus \nu_{\mathcal{P}}(p)
\end{aligned}
$$

e

$$
L_{\mathcal{P}}(p)=p+\mathbf{F}_{\mathcal{P}}(p)
$$

para cada $p \in M$.

Proposição 2.2.4. Seja $M$ uma subvariedade isoparamétrica de $\mathbf{E}^{n}$. Com a notação acima temos que

a. $\mathfrak{D}_{\mathcal{P}}$ é uma distribuição auto-paralela de $M$ e portanto tem folhas totalmente geodésicas em $M$. 
b. $\mathfrak{D}_{\mathcal{P}}$ é invariante pelos operadores de forma de $M$.

c. $S e S_{\mathcal{P}}(p)$ denota a folha de $\mathfrak{D}_{\mathcal{P}}$ passando por $p$, então $S_{\mathcal{P}}(p)$ é uma subvariedade isoparamétrica substancial do espaço Euclidiano $L_{\mathcal{P}}(p)$. E para cada $q \in S_{\mathcal{P}}(p)$ temos $L_{\mathcal{P}}(p)=L_{\mathcal{P}}(q)$.

Demonstração. a. Sejam $i, j, k \in\{1, \ldots, g\}$ tais que $\mathbf{n}_{i}\left(p_{0}\right), \mathbf{n}_{j}\left(p_{0}\right) \in \mathcal{P}$ e $\mathbf{n}_{k}\left(p_{0}\right) \notin \mathcal{P}$. Se $i \neq j$, temos que $\mathbf{n}_{j}\left(p_{0}\right)-\mathbf{n}_{k}\left(p_{0}\right)$ e $\mathbf{n}_{i}\left(p_{0}\right)-\mathbf{n}_{j}\left(p_{0}\right)$ são linearmente independentes. Agora, o transporte paralelo é uma isometria linear de $\nu_{p} M$ sobre $\nu_{q} M$, para todo par $p, q \in M$. Então $\mathbf{n}_{j}(p)-\mathbf{n}_{k}(p) \neq 0$ e se $i \neq j, \mathbf{n}_{j}(p)-\mathbf{n}_{k}(p)$ e $\mathbf{n}_{i}(p)-\mathbf{n}_{j}(p)$ são linearmente independentes para todo $p \in M$. Trocando os índices na equação (2.7) obtemos $\left\langle\nabla_{X_{i}} X_{j}, X_{k}\right\rangle\left(\mathbf{n}_{j}-\mathbf{n}_{k}\right)=\left\langle\nabla_{X_{k}} X_{j}, X_{i}\right\rangle\left(\mathbf{n}_{j}-\mathbf{n}_{i}\right)$, assim

$$
\left\langle\nabla_{X_{i}} X_{j}, X_{k}\right\rangle=0
$$

Logo se $X=\sum_{\mathbf{n}_{i}\left(p_{0}\right) \in \mathcal{P}} X_{i}$ e $Y=\sum_{\mathbf{n}_{j}\left(p_{0}\right) \in \mathcal{P}} Y_{j}$ são duas seções de $\mathfrak{D}_{\mathcal{P}}$ e $Z$ é uma seção de $E_{k}$, com $\mathbf{n}_{k}\left(p_{0}\right) \notin \mathcal{P}$, temos que

$$
\left\langle\nabla_{X} Y, Z\right\rangle=\sum_{\mathbf{n}_{i}\left(p_{0}\right), \mathbf{n}_{j}\left(p_{0}\right) \in \mathcal{P}}\left\langle\nabla_{X_{i}} Y_{j}, Z\right\rangle=0
$$

Assim $\mathfrak{D}_{\mathcal{P}}$ é auto paralela.

b. Como $R^{\perp}=0$, então os operadores de forma de $M$ comutam, logo preservam as distribuições de curvatura. Em particular deixam $\mathfrak{D}_{\mathcal{P}}$ invariante.

c. Seja $p \in M$ fixo. Dado $q \in S_{\mathcal{P}}(p)$ seja $\gamma[0,1] \longrightarrow S_{\mathcal{P}}(p)$ uma curva suave por partes com $\gamma(0)=p$ e $\gamma(1)=q$. Sejam $u \in \mathfrak{D}_{\mathcal{P}}(p)^{\perp} \cap T_{p} M$ um vetor normal a $S_{\mathcal{P}}(p)$, vista como subvariedade de $M$, e $U(t)$ o transporte paralelo de $u$ ao longo de $\gamma$. Como $S_{\mathcal{P}}(p) \subset M$ é totalmente geodésica temos que $\nabla_{\gamma^{\prime}(t)} U(t)=0$ e do item b. e da observação (2.1.3), temos que $\alpha\left(\gamma^{\prime}(t), U(t)\right)=0$. Logo pela formula de Gauss tem-se

$$
U^{\prime}(t)=\nabla_{\gamma^{\prime}(t)} U(t)+\alpha\left(\gamma^{\prime}(t), U(t)\right)=0,
$$

para todo $t \in[0,1]$. Então $U$ é constante e como $u$ é arbitrário

$$
\mathfrak{D}_{\mathcal{P}}(p)^{\perp} \cap T_{p} M=\mathfrak{D}_{\mathcal{P}}(q)^{\perp} \cap T_{q} M .
$$


Por outro lado

$$
\frac{d}{d t}\langle\gamma(t)-p, U(t)\rangle=\left\langle\gamma^{\prime}(t), U(t)\right\rangle+\left\langle\gamma(t), U^{\prime}(t)\right\rangle=0,
$$

para todo $t \in[0,1]$. Logo $\langle\gamma-p, U\rangle$ é constante e assim $\langle q-p, v\rangle=0$. Como $q \in S_{\mathcal{P}}(p)$ é arbitrário obtemos

$$
S_{\mathcal{P}}(p) \subset p+\left(\mathfrak{D}_{\mathcal{P}}(p)^{\perp} \cap T_{p} M\right)^{\perp}=p+\mathfrak{D}_{\mathcal{P}}(p) \oplus \nu_{p} M
$$

e de $(2.8)$

$$
\mathfrak{D}_{\mathcal{P}}(p) \oplus \nu_{p} M=\mathfrak{D}_{\mathcal{P}}(q) \oplus \nu_{q} M,
$$

para todo $q \in S_{\mathcal{P}}(p)$.

Consideremos $S_{\mathcal{P}}(p)$ como subvariedade de $p+\mathfrak{D}_{\mathcal{P}}(p) \oplus \nu_{p} M$. Pelo item b. los operadores de forma de $M$ preservam $\mathfrak{D}_{\mathcal{P}}$, logo para cada $q \in S_{\mathcal{P}}(p)$ e cada $v \in \nu_{q} M$ o operador de forma de $S_{\mathcal{P}}(p)$ na direção de $v$ é $\left.A_{v}\right|_{\mathfrak{D}_{\mathcal{P}}(q)}$, o qual é nulo para $v \in \nu_{\mathcal{P}}^{\perp} \cap \nu_{p} M$. Assim, por um razonamento análogo ao usado acima

$$
\mathbf{F}_{\mathcal{P}}(p)=\mathbf{F}_{\mathcal{P}}(q)
$$

para todo $q \in S_{\mathcal{P}}(p)$ e

$$
S_{\mathcal{P}}(p) \subset L_{\mathcal{P}}(p) .
$$

De (2.10) segue que $L_{\mathcal{P}}(p)=L_{\mathcal{P}}(q)$, para todo $q \in S_{\mathcal{P}}(p)$. Consideremos $S_{\mathcal{P}}(p)$ como subvariedade de $L_{\mathcal{P}}(p)$ denotemos seu operador de forma por $\hat{A}$, de novo pelo item b. se tem-se

$$
\hat{A}_{v}=\left.A_{v}\right|_{\mathfrak{D}_{\mathcal{P}}(q)}
$$

para todo $q \in S_{\mathcal{P}}(p)$ e todo $v \in \nu_{\mathcal{P}}(q)$. Suas normais de curvatura são $\left.\mathbf{n}_{j}\right|_{S_{\mathcal{P}}(p)}$, para $j \in\{i$ : $\left.\mathbf{n}_{i}\left(p_{0}\right) \in \mathcal{P}\right\}$. Assim $S_{\mathcal{P}}(p)$ é uma subvariedade isoparamétrica e substancial do espaço Euclidiano $L_{\mathcal{P}}(p)$.

Observação 2.2.5. Note que no item c. da demonstração acima está implícito que $S_{\mathcal{P}}(p)$ é uma subvariedade isoparamétrica de $p+\mathfrak{D}_{\mathcal{P}}(p) \oplus \nu_{p} M$, onde $p+\mathfrak{D}_{\mathcal{P}}(p) \oplus \nu_{p} M=q+\mathfrak{D}_{\mathcal{P}}(q) \oplus \nu_{q} M$, para todo $q \in S_{\mathcal{P}}(p)$. Além disso $\sum_{\mathbf{n}_{i}\left(p_{0}\right) \in \mathcal{P}} \mathbf{R}_{i}(p)$ é o primeiro espaço normal de $S_{\mathcal{P}}(p)$ vista como subvariedade do espaço Euclidiano $p+\mathfrak{D}_{\mathcal{P}}(p) \oplus \nu_{p} M$. 
Corolário 2.2.6. Seja $M$ uma subvariedade isoparamétrica de $\mathbf{E}^{n}$. Então as distribuições de curvatura de $M$ são auto paralelas e portanto têm folhas totalmente geodésicas. Dado $p \in M$, seja $S_{i}(p)$ a folha de $E_{i}$ passando por $p, i \in\{1, \ldots, g\}$. Ainda

a. Se $\mathbf{n}_{i}(p)=0$, então $S_{i}(p)$ é um aberto de $p+E_{i}(p)=q+E_{i}(q)$, para todo $q \in S_{i}(p)$.

b. Se $\mathbf{n}_{i}(p) \neq 0$, então $S_{i}(p)$ é um aberto de uma hiperesfera de centro em $p+\frac{1}{\left|\mathbf{n}_{i}(p)\right|^{2}} \mathbf{n}_{i}(p)$ e raio $\frac{1}{\left|\mathbf{n}_{i}(p)\right|}$ no espaço afim $p+E_{i}(p) \oplus \mathbf{R n}_{i}(p)=q+E_{i}(q) \oplus \mathbf{R n}_{i}(q)$,para todo $q \in S_{i}(p)$.

Demonstração. Fazendo $\mathcal{P}=\left\{\mathbf{n}_{i}\left(p_{0}\right)\right\}$, da proposição anterior temos que $\mathfrak{D}_{\mathcal{P}}=E_{i}$ é auto-paralela. Também que $S_{i}(p)$ é uma subvariedade isoparamétrica de $L_{i}(p)=p+E_{i}(p) \oplus \mathbf{R n}_{i}(p)$ (neste caso $S_{i}(p)$ é uma subvariedade totalmente umbílica de $\left.L_{i}(p)\right)$, com normal de curvatura $\left.\mathbf{n}_{i}\right|_{S_{i}(p)}$.

a. Se $\mathbf{n}_{i}(p)=0$, então $S_{i}(p) \subset p+E_{i}(p)$.

b. Se $\mathbf{n}_{i}(p) \neq 0$, seja $r=\frac{1}{\left|\mathbf{n}_{i}(p)\right|}$. Então dado $q \in S_{i}(p)$, sejam $\gamma:[0,1] \longrightarrow S_{i}(p)$ uma curva suave por partes com $\gamma(0)=p$ e $\gamma(1)=q$. Como $\mathbf{n}_{i}$ é paralelo temos que

$$
\begin{aligned}
\frac{d}{d t}\left(\gamma(t)+r^{2} \mathbf{n}_{i}(\gamma(t))\right) & =\gamma^{\prime}(t)-r^{2}\left\langle\mathbf{n}_{i}(\gamma(t)), \mathbf{n}_{i}(\gamma(t))\right\rangle \gamma^{\prime}(t) \\
& =\gamma^{\prime}(t)-r^{2}\left\langle\mathbf{n}_{i}(\gamma(t)), \mathbf{n}_{i}(\gamma(t))\right\rangle \gamma^{\prime}(t) \\
& =\gamma^{\prime}(t)-\gamma^{\prime}(t) \\
& =0,
\end{aligned}
$$

para todo $t \in[0,1]$. Logo $\gamma+r^{2} \mathbf{n}_{i} \circ \gamma$ é constante, em particular

$$
p+r^{2} \mathbf{n}_{i}(p)=\gamma(0)+r^{2} \mathbf{n}_{i}(\gamma(0))=\gamma(1)+r^{2} \mathbf{n}_{i}(\gamma(1))=q+r^{2} \mathbf{n}_{i}(q) .
$$

Como $q$ é arbitrário e $\left|\mathbf{n}_{i}\right|$ é constante, temos que $\left|q-\left(p+r^{2} \mathbf{n}_{i}(p)\right)\right|=r$, para todo $q \in S_{i}(p)$

Observação 2.2.7. Seja $M$ uma subvariedade isoparamétrica do espaço Euclidiano.

a. Sejam $p, q \in M$ e $\tau: \nu_{p} M \longrightarrow \nu_{q} M$ o transporte paralelo. Se $v \in \mathcal{N}_{p}^{\perp}$, da observação (2.2.1) tem-se

$$
0=\left\langle\mathbf{n}_{i}(p), v\right\rangle=\left\langle\tau \mathbf{n}_{i}(p), \tau v\right\rangle=\left\langle\mathbf{n}_{i}(q), \tau v\right\rangle
$$


para todo $i \in\{1, \ldots, g\}$. Logo $\mathcal{N}^{\perp}$ é preservado pelo transporte paralelo.

b. Seja $p \in M$ fixo. Se $v \in \nu_{p} M \operatorname{com}\left\langle\mathbf{n}_{i}(p), v\right\rangle=0$, para todo $i \in\{1, \ldots, g\}$, do item anterior e do item a. na demonstração do teorema (1.5.1) tem-se $M \subset p+v^{\perp}$.

c. Suponhamos que $M$ é substancial. Então pelo item anterior e a observação (2.2.1) tem-se $\nu_{p} M=$ $\sum_{i} \mathbf{R n}_{i}(p)$, para todo $p \in M$.

d. Suponhamos que $M$ é substancial e compacta. Então de b. $M$ tem uma normal de curvatura nula se e somente se sua correspondente curvatura principal é nula. Neste caso seja $E_{0}$ a distribuição de nulidade. Como $M$ é completa as folhas de $E_{0}$ são completas logo do item a. do corolário anterior são subespaços afins do espaço Euclidiano, os quais são triviais já que $M$ é limitada. Logo $M$ tem normais de curvatura não nulas.

Observação 2.2.8. Seja $M$ uma subvariedade isoparamétrica do espaço Euclidiano. O posto de $M$ é o número máximo de normais de curvatura linearmente independentes. Do item a. da observação acima, do teorema (1.5.1) e do item c. da mesma observação podemos considerar, $M \subset \mathbf{E}^{n}$ com $n=m+l$ onde $l$ é o posto de $M$. Em diante somente consideraremos subvariedades isoparamétricas substanciais do Espaço Euclidiano.

\subsection{O grupo de Coxeter}

Um subgrupo grupo finito e essencial* do grupo ortogonal de um espaço vetorial finito dimensional com produto interno gerado por um conjunto de reflexões é chamado grupo de Coxeter. O posto do grupo de Coxeter é o número máximo de vetores linearmente independentes do conjunto finito de vetores tais que as reflexões são feitas em relação aos seus espaços ortogonais. Dada uma subvariedade isoparamétrica do espaço Euclidiano podemos associar-lhe um grupo de Coxeter. Para isto consideraremos reflexões de subespaços afins do espaço Euclidiano em relação a certos hiperplanos; a saber, os espaços normais afins e os hiperplanos focais, respectivamente. Este grupo será uma ferramenta importante no estudo da geometria da subvariedade. Em particular o posto da subvariedade coincidirá com o posto do respectivo grupo de Coxeter.

\footnotetext{
*Essencial no sentido de que o conjunto dos pontos fixos do grupo é a origem. Na verdade os grupos de Coxeter em geral não são só finitos mas sim discretos. No nosso caso estudaremos grupos de Coxeter finitos.
} 
Sejam $M \subset \mathbf{E}^{n}$ uma subvariedade isoparamétrica e $\mathbf{n}_{1}, \ldots, \mathbf{n}_{g}$ suas normais de curvatura não nulas. Dado $p \in M$ para $i \in\{1, \ldots, g\}$ definimos o $i$-ésimo hiperplano focal de $M$ em $p, l_{i}(p)$ por

$$
l_{i}(p)=\left\{p+v: v \in \nu_{p} M \quad e\left\langle\mathbf{n}_{i}(p), v\right\rangle=1\right\} .
$$

Temos que o conjunto focal de $M$ em $p$ é a reunião dos hiperplanos focais em $p$.

No que resta desta seção utilizaremos a seguinte notação. Dados $p, q \in M \tau_{p}^{q}: \nu_{p} M \longrightarrow \nu_{q} M$ denotará o transporte paralelo (ao longo de uma curva suave por partes unindo $p$ e $q$ ) em relação à conexão normal de M. Definimos o transporte paralelo afim $\hat{\tau}_{p}^{q}: p+\nu_{p} M \longrightarrow q+\nu_{q} M$ por

$$
\hat{\tau}_{p}^{q}(p+v)=q+\tau_{p}^{q} v
$$

para todo $v \in \nu_{p} M . R_{i}^{p}$ e $\rho_{i}^{p}$ denotarão as reflexões de $\nu_{p} M$ e $p+\nu_{p} M$ em relação aos hiperplanos $\mathbf{n}_{i}(p)^{\perp} \cap \nu_{p} M$ e $l_{i}(p)$, respectivamente, para $p \in M$ e $i \in\{1, \ldots, g\}$. Ademais, consideraremos $M$ completa, assim as folhas das distribuições de curvatura associadas a normais de curvatura não nulas são hiperesferas de subespaços afins do espaço Euclidiano. $a_{i}: S_{i}(p) \longrightarrow S_{i}(p)$ denotará a aplicação antípoda, isto é $a_{i}(q)$ é o outro ponto de interseç̧ão entre a reta unindo $q$ ao centro de $S_{i}(p)$ e $S_{i}(p)$, para todo $q \in S_{i}(p)$.

Lema 2.3.1. Com a notação acima temos que para todo $i \in\{1, \ldots, g\}$

a. $\rho_{i}^{p}(p)=a_{i}(p)$

b. $\rho_{i}^{p}=\hat{\tau}_{p}^{a_{i}(p)}$

c. $\rho_{i}^{p}\left(l_{j}(p)\right)=l_{j}\left(a_{i}(p)\right)$, para todo $j \in\{1, \ldots, g\}$ e portanto $\rho_{i}^{p}\left(F_{M}(p)\right)=F_{M}\left(a_{i}(p)\right)$

Demonstração. Com efeito,

a. Temos que

$$
\begin{aligned}
l_{i}(p) & =p+\left\{v \in \nu_{p} M:\left\langle\mathbf{n}_{i}(p), v\right\rangle=1\right\} \\
& =p+\left\{v \in \nu_{p} M:\left\langle\mathbf{n}_{i}(p), v-\frac{1}{\left|\mathbf{n}_{i}(p)\right|^{2}} \mathbf{n}_{i}(p)\right\rangle=0\right\} \\
& =p+\left(\frac{1}{\left|\mathbf{n}_{i}(p)\right|^{2}} \mathbf{n}_{i}(p)+\mathbf{n}_{i}(p)^{\perp} \cap \nu_{p} M\right) .
\end{aligned}
$$


$R_{i}^{p}$ está dada por

$$
R_{i}^{p} v=v-2 \frac{\left\langle\mathbf{n}_{i}(p), v\right\rangle}{\left|\mathbf{n}_{i}(p)\right|^{2}} \mathbf{n}_{i}(p),
$$

para todo $v \in \nu_{p} M$. Assim

$$
\begin{aligned}
\rho_{i}^{p}(p+v) & =p+\frac{1}{\left|\mathbf{n}_{i}(p)\right|^{2}} \mathbf{n}_{i}(p)+R_{i}^{p}\left(v-\frac{1}{\left|\mathbf{n}_{i}(p)\right|^{2}} \mathbf{n}_{i}(p)\right) \\
& =p+v+2 \frac{\left(1-\left\langle\mathbf{n}_{i}(p), v\right\rangle\right)}{\left|\mathbf{n}_{i}(p)\right|^{2}} \mathbf{n}_{i}(p),
\end{aligned}
$$

para todo $v \in \nu_{p} M$. Agora seja $r(t)=(1-t) p+t\left(p+\frac{1}{\left|\mathbf{n}_{i}(p)\right|^{2}} \mathbf{n}_{i}(p)\right), t \in \mathbf{R}$, a reta unindo $\mathrm{p}$ ao centro de $S_{i}(p)$, com $r(0)=p$ e $r(1)=p+\frac{1}{\mathbf{n}_{i}(p)^{2}} \mathbf{n}_{i}(p)$. Então $a_{i}(p)=r(2)=p+\frac{2}{\left|\mathbf{n}_{i}(p)\right|^{2}} \mathbf{n}_{i}(p)=\rho_{i}^{p}(p)$.

b. Pela observação (2.2.5) temos que $S_{i}(p)$ é uma subvariedade totalmente umbílica de $p+E_{i}(p) \oplus \nu_{p} M$ e também que o transporte paralelo normal de $S_{i}(p)$, vista como subvariedade deste espaço afim, coincide com o transporte paralelo normal de $M$ restrito a $S_{i}(p)$. Então

$$
\mathbf{n}_{i}\left(a_{i}(p)\right)=\tau_{p}^{a_{i}(p)} \mathbf{n}_{i}(p)=-\mathbf{n}_{i}(p)
$$

e $\tau_{p}^{a_{i}(p)} v=v$, se $v \in \nu_{p} M \operatorname{com}\left\langle\mathbf{n}_{i}(p), v\right\rangle=0$. Portanto $\tau_{p}^{a_{i}(p)}$ coincide com $R_{i}^{p}$, assim da equação (2.13) tem-se

$$
\begin{aligned}
\rho_{i}^{p}(p+v) & =p+\frac{2}{\left|\mathbf{n}_{i}(p)\right|^{2}} \mathbf{n}_{i}(p)+R_{i}^{p} v \\
& =a_{i}(p)+\tau_{p}^{a_{i}(p)} v \\
& =\hat{\tau}_{p}^{a_{i}(p)}(p+v),
\end{aligned}
$$

para todo $v \in \nu_{p} M$, 
c. Da equação acima temos que

$$
\begin{aligned}
\rho_{i}^{p}\left(l_{j}(p)\right) & =\left\{\rho_{i}^{p}(p+v):\left\langle\mathbf{n}_{j}(p), v\right\rangle=1\right\} \\
& =\left\{a_{i}(p)+\tau_{p}^{a_{i}(p)} v:\left\langle\mathbf{n}_{j}(p), v\right\rangle=1\right\} \\
& =\left\{a_{i}(p)+\tau_{p}^{a_{i}(p)} v:\left\langle\mathbf{n}_{j}\left(a_{i}(p)\right), \tau_{p}^{a_{i}(p)} v\right\rangle=1\right\} \\
& =l_{j}\left(a_{i}(p)\right)
\end{aligned}
$$

Consideremos os $g$ campos paralelos $\xi_{i}=\frac{2}{\left|\mathbf{n}_{i}\right|^{2}} \mathbf{n}_{i}$ e as respectivas projeções $\pi_{i}: M \longrightarrow M_{\xi_{i}}$. Temos que $\pi_{i}(p)=p+\frac{2}{\left|\mathbf{n}_{i}(p)\right|^{2}} \mathbf{n}_{i}(p)=a_{i}(p)$. Logo $M_{\xi_{i}}=M$. Portanto da proposição (2.1.6) item b. e do item c. do lema acima temos que

$$
\begin{aligned}
F_{M}(p) & =F_{M_{\xi_{i}}}\left(\pi_{i}(p)\right) \\
& =F_{M}\left(a_{i}(p)\right) \\
& =\rho_{i}^{p}\left(F_{M}(p)\right),
\end{aligned}
$$

para todo $p \in M$.

Note que para cada $i \in\{1, \ldots, g\}$ fazendo $\sigma_{i}=\sigma$ na equação (2.5) como $M=M_{\xi_{i}}$ obtemos

$$
\mathbf{n}_{\sigma_{i}(j)}\left(a_{i}(p)\right)=\frac{1}{1-\left\langle\mathbf{n}_{j}(p), \xi_{i}(p)\right\rangle} \mathbf{n}_{j}(p)
$$

para todo $j \in\{1, \ldots, g\}$.

Observação 2.3.2. Note que a existência das permutações $\sigma_{i}$ impõe uma forte restrição às multiplicidades das curvaturas principais, a saber

$$
m_{j}=\operatorname{dim}\left(E_{j}\right)=\operatorname{dim}\left(E_{\sigma_{i}(j)}\right)=m_{\sigma_{i}(j)} .
$$


Lema 2.3.3. As reflexões $\rho_{1}^{p}, \ldots, \rho_{g}^{p}$ permutam os hiperplanos focais de $M$ em $p$. Ainda $\rho_{i}^{p}\left(l_{j}(p)\right)=$ $l_{\sigma_{i}(j)}(p)$

Demonstração. Do item c. do lema (2.3.1) e das equações (2.16) e (2.14) tem-se

$$
\begin{aligned}
\rho_{i}^{p}\left(l_{j}(p)\right) & =l_{j}\left(a_{i}(p)\right) \\
& =\left\{a_{i}(p)+v:\left\langle\mathbf{n}_{j}\left(a_{i}(p)\right), v\right\rangle=1\right\} \\
& =\left\{p+\xi_{i}(p)+v:\left\langle-\mathbf{n}_{j}(p), v\right\rangle=1\right\} \\
& =\left\{p+w:-\left\langle\mathbf{n}_{j}(p), w-\xi_{i}(p)\right\rangle=1\right\} \\
& =\left\{p+w: \frac{\left\langle-\mathbf{n}_{j}(p), w\right\rangle}{1-\left\langle\mathbf{n}_{j}(p), \xi_{i}(p)\right\rangle}=1\right\} \\
& =\left\{p+w:\left\langle-\mathbf{n}_{\sigma_{i}(j)}\left(a_{i}(p)\right), w\right\rangle=1\right\} \\
& =\left\{p+w:\left\langle\mathbf{n}_{\sigma_{i}(j)}(p), w\right\rangle=1\right\} \\
& =l_{\sigma_{i}(j)}(p) .
\end{aligned}
$$

Lema 2.3.4. Seja $M$ uma subvariedade isoparamétrica do espaço Euclidiano. Dado $p \in M$ seja $\tilde{W}^{p}$ o subgrupo de $G L\left(\nu_{p} M\right)$ gerado pelas reflexões $R_{1}^{p}, \ldots, R_{g}^{p}$. Então $\tilde{W}^{p}$ é um grupo de Coxeter finito.

Demonstração. Um ponto de $\nu_{p} M$ é fixado por todo elemento de $\tilde{W}^{p}$ se e somente se é fixado pelas reflexões $R_{1}^{p}, \ldots, R_{g}^{p}$. Logo a intersecção dos conjuntos de pontos fixos de todos os elementos de $\tilde{W}^{p}$ é a intersecção dos hiperespaços de $\nu_{p} M$ ortogonais às normais de curvatura. Agora, como as normais de curvatura geram $\nu_{p} M$, temos que $\nu_{p} M=\left(\cap_{i=1}^{g} \nu_{p} M \cap \mathbf{n}_{i}(p)^{\perp}\right)^{\perp}$. Logo $\tilde{W}^{p}$ é essencial.

De outro lado, como $R_{i}^{p}=\tau_{p}^{a_{i}(p)}$ (demonstração do item b. do lema (2.3.1)), a equação (2.16) pode ser reescrita assim

$$
R_{i}^{p} \mathbf{n}_{\sigma_{i}(j)}(p)=\left(1-\frac{2\left\langle\mathbf{n}_{j}(p), \mathbf{n}_{i}(p)\right\rangle}{\left|\mathbf{n}_{i}(p)\right|^{2}}\right)^{-1} \mathbf{n}_{j}(p)
$$

então

$$
\left|\mathbf{n}_{\sigma_{i}(j)}(p)\right|=\left|1-\frac{2\left\langle\mathbf{n}_{j}(p), \mathbf{n}_{i}(p)\right\rangle}{\left|\mathbf{n}_{i}(p)\right|^{2}}\right|^{-1}\left|\mathbf{n}_{j}(p)\right|,
$$


e como $\left(R_{i}^{p}\right)^{2}=i d_{\nu_{p} M}$ temos que

$$
R_{i}^{p}\left(\frac{1}{\left|\mathbf{n}_{j}(p)\right|} \mathbf{n}_{j}(p)\right)=\frac{{ }_{-}^{+} 1}{\left|\mathbf{n}_{\sigma_{i}(j)}(p)\right|} \mathbf{n}_{\sigma_{i}(j)}(p) .
$$

Logo as reflexões $R_{1}^{p}, \ldots, R_{g}^{p}$ permutam o conjunto

$$
\Delta_{p}=\left\{\frac{{ }_{-}^{+} 1}{\left|\mathbf{n}_{i}(p)\right|} \mathbf{n}_{i}(p): i=1, \ldots, g\right\},
$$

portanto existe um homomorfismo de $\tilde{W}^{p}$ no grupo de permutações de $\Delta_{p}$. Este homomorfismo é injetor já que se um elemento de $\tilde{W}^{p}$ restrito a $\Delta_{p}$ é a identidade, então é a identidade de $\nu_{p} M$ (as normais de curvatura geram $\left.\nu_{p} M\right)$. Segue que $\tilde{W}^{p}$ é finito.

Teorema 2.3.5 (Terng). Seja $M$ uma subvariedade isoparamétrica compacta do espaço Euclidiano. Então o subgrupo $W^{p}$, do grupo de isometrias de $p+\nu_{p} M$, gerado pelas reflexões $\rho_{1}^{p}, \ldots, \rho_{g}^{p}$ é um grupo de Coxeter, para cada $p \in M$. Dizemos que $W^{p}$ é o grupo de Coxeter de $M$ em $p$.

Demonstração. Como $M$ é compacta da observação (2.2.7) item d. segue que suas normais de curvatura são não nulas. Seja $\varphi: W^{p} \longrightarrow \tilde{W}^{p}$ a aplicação dada por $\varphi\left(\rho_{i_{1}}^{p} \cdots \rho_{i_{s}}^{p}\right)=R_{i_{1}}^{p} \cdots R_{i_{s}}^{p}$. Vejamos que $\varphi$ é um homomorfismo injetor. Com efeito, pela equação (2.15) temos que para todo $v \in \nu_{p} M$

$$
\rho_{i_{1}}^{p} \cdots \rho_{i_{s}}^{p}(p+v)=p+\xi_{\left(i_{1}, \ldots, i_{s}\right)}(p)+R_{i_{1}}^{p} \cdots R_{i_{s}}^{p} v
$$

onde

$$
\xi_{\left(i_{1}, \ldots, i_{s}\right)}(p)=\xi_{i_{1}}(p)+R_{i_{1}}^{p} \xi_{i_{2}}+R_{i_{1}}^{p} R_{i_{2}}^{p} \xi_{i_{3}}+\ldots+R_{i_{1}}^{p} \cdots R_{i_{s-1}}^{p} \xi_{i_{s}}
$$

e $\xi_{i_{j}}=\frac{2}{\left|\mathbf{n}_{i_{j}}\right|^{2}} \mathbf{n}_{i_{j}}$, com $j \in\{1, \ldots, s\}$. Assim $\varphi(\rho)=d \rho_{x}$, para todo $x \in \nu_{p} M$ e para cada $\rho \in W^{p}$, portanto $\varphi$ está bem definido e é um homomorfismo de grupos. Agora suponhamos que $\varphi\left(\rho_{i_{1}}^{p} \cdots \rho_{i_{s}}^{p}\right)=$ $i d_{\nu_{p} M}$. Como as reflexões $R_{1}^{p}, \ldots R_{g}^{p}$ permutam $\Delta_{p}$, também permutam os hiperespaços ortogonais às normais de curvatura, então $\sigma_{i_{1}} \cdots \sigma_{i_{s}}$ é a permutação identidade. De outro lado as reflexões $\rho_{1}^{p}, \ldots, \rho_{g}^{p}$ permutam os hiperplanos focais, assim dado $p+v \in l_{j}(p)$ temos que $\rho_{i_{1}}^{p} \cdots \rho_{i_{s}}^{p}(p+v) \in$ $l_{\sigma_{i_{1}} \cdots \sigma_{i_{s}}(j)}(p)=l_{j}(p)$. Então da equação (2.17) tem-se

$$
1=\left\langle\mathbf{n}_{j}(p), \xi_{\left(i_{1}, \ldots, i_{s}\right)}(p)+v\right\rangle=\left\langle\mathbf{n}_{j}(p), \xi_{\left(i_{1}, \ldots, i_{s}\right)}(p)\right\rangle+1
$$


para todo $j \in\{1, \ldots, g\}$. Logo $\xi_{\left(i_{1}, \ldots, i_{s}\right)}(p)=0$ e portanto $\rho_{i_{1}}^{p} \cdots \rho_{i_{s}}^{p}$ é a identidade de $p+\nu_{p} M$. Ou seja $\varphi$ é injetor. Pelo lema anterior $\tilde{W}^{p}$ é finito, então $W^{p}$ é finito.

Um ponto de $p+\nu_{p} M$ é fixado por todo elemento de $W^{p}$ se e somente se é fixado por $\rho_{i}^{p}$ para todo $i \in\{1, \ldots, g\}$. Assim a intersecção dos conjuntos de pontos fixos dos elementos de $W^{p}$ é a intersecção dos hiperplanos focais de $M$ em $p$. Dado $x_{0} \in \nu_{p} M$ fixo, consideremos a função

$$
f(x)=\max \left\{\frac{1}{2}\left|\rho^{\prime}\left(x_{0}\right)-x\right|^{2}: \rho^{\prime} \in W^{p}\right\}
$$

$x \in \nu_{p} M . f$ é uma função estritamente convexa, já que é o máximo de um conjunto finito de funções estritamente convexas, logo tem um único ponto de mínimo $x_{1}$ (isto é, $x_{1}$ é o centro de massa da órbita de $x_{0}$ dada pela ação de $W^{p}$ sobre $\left.p+\nu_{p} M\right)$. Dado $\rho \in W^{p}$, sabemos que $\rho$ preserva a distância assim

$$
\begin{aligned}
f\left(\rho\left(x_{1}\right)\right) & =\max \left\{\frac{1}{2}\left|\rho^{\prime}\left(x_{0}\right)-\rho\left(x_{1}\right)\right|^{2}: \rho^{\prime} \in W^{p}\right\} \\
& =\max \left\{\frac{1}{2}\left|\rho^{-1} \circ \rho^{\prime}\left(x_{0}\right)-x_{1}\right|^{2}: \rho^{\prime} \in W^{p}\right\} \\
& =f\left(x_{1}\right)
\end{aligned}
$$

assim $\rho\left(x_{1}\right)$ é ponto de mínimo de $f$, ou seja $\rho\left(x_{1}\right)=x_{1}$. Como $\rho$ é arbitrário segue que $x_{1} \in \cap_{i=1}^{g} l_{i}(p)$. Por último suponhamos que existem $p+v_{1}, p+v_{2} \in \cap_{i=1}^{g} l_{i}(p)$. Assim $\left\langle\mathbf{n}_{j}(p), v_{1}-v_{2}\right\rangle=\left\langle\mathbf{n}_{j}(p), v_{1}\right\rangle-$ $\left\langle\mathbf{n}_{j}(p), v_{2}\right\rangle=1-1=0$, para todo $j \in\{1, \ldots, g\}$, logo $v_{1}-v_{2}=0$ e portanto $p+v_{1}=p+v_{2}$. Segue que existe um único ponto $b_{p} \in \cap_{i=1}^{g} l_{i}(p)$, ou seja $W^{p}$ é essencial.

Observação 2.3.6. Note que se $p \in M$ a equação (2.15) implica que

$$
\rho_{i}^{p}(x)=R_{i}^{p}\left(x-b_{p}\right)+b_{p},
$$

para todo $x \in p+\nu_{p} M$ e cada $i \in\{1, \ldots, g\}$. Logo

$$
\rho(x)=R\left(x-b_{p}\right)+b_{p},
$$

para todo $x \in p+\nu_{p} M$, onde $\varphi(\rho)=R$. 
De outro lado

$$
l_{i}(p)=\left\{x \in p+\nu_{p} M:\left\langle\frac{1}{\left|\mathbf{n}_{i}(p)\right|} \mathbf{n}_{i}(p), x-b_{p}\right\rangle=0\right\},
$$

para todo $i \in\{1, \ldots, g\}$. Segue que $W^{p}$ e $\tilde{W}^{p}$ são isomorfos.

Corolário 2.3.7. Seja $M$ uma subvariedade isoparamétrica compacta do espaço Euclidiano. Então existe um campo normal paralelo $\zeta$ tal que $\left\langle\zeta, \mathbf{n}_{i}\right\rangle=1$. para todo $i \in\{1, \ldots, g\}$.

Demonstração. Com efeito, seja $\zeta_{p}=b_{p}-p$,para todo $p \in M$.

Lema 2.3.8. Os grupos de Coxeter em relação a cada ponto de $M$ são conjugados entre si.

Demonstração. Com efeito. Se $i \in\{1, \ldots, g\}$ fixo, da proposição (2.1.6) temos que

$$
\nu_{p} M=\nu_{\pi_{i}(p)} M_{\xi_{i}}=\nu_{\pi_{i}(p)} M
$$

$\mathrm{e}$

$$
p+\nu_{p} M=\pi_{i}(p)+\nu_{\pi_{i}(p)} M .
$$

Do lema (2.1.4) $\tau_{p}^{q}=\tau_{a_{i}(p)}^{a_{i}(q)}$. Agora, se $v \in \nu_{p} M$ e $q \in M$, tem-se

$$
\begin{aligned}
\hat{\tau}_{p}^{q}(p+v) & =q+\tau_{p}^{q} v \\
& =a_{i}(q)-\xi_{i}(q)+\tau_{a_{i}(p)}^{a_{i}(q)} v \\
& =a_{i}(q)+\xi_{i}\left(a_{i}(q)\right)+\tau_{a_{i}(p)}^{a_{i}(q)} v \\
& =a_{i}(q)+\tau_{a_{i}(p)}^{a_{i}(q)}\left(\xi_{i}\left(a_{i}(p)\right)+v\right) \\
& =a_{i}(q)+\tau_{a_{i}(p)}^{a_{i}(q)}\left(-\xi_{i}(p)+v\right) \\
& =\hat{\tau}_{a_{i}(p)}^{a_{i}(q)}\left(a_{i}(p)+v-\xi_{i}(p)\right) \\
& =\hat{\tau}_{a_{i}(p)}^{a_{i}(q)}(p+v) .
\end{aligned}
$$


Portanto, do item b. do lema (2.3.1) obtemos

$$
\begin{aligned}
\hat{\tau}_{p}^{q} \rho_{i}^{p} \hat{\tau}_{q}^{p} & =\hat{\tau}_{a_{i}(p)}^{a_{i}(q)} \hat{\tau}_{p}^{a_{i}(p)} \hat{\tau}_{q}^{p} \\
& =\hat{\tau}_{q}^{a_{i}(q)} \\
& =\rho_{i}^{q} .
\end{aligned}
$$

\subsubsection{A câmera de Weyl}

Seja $M \subset \mathbf{E}^{n}$ uma subvariedade isoparamétrica. Fixando $p \in M$, consideremos um ponto $x$ no complementar $F_{M}(p)^{c}$ de $F_{M}(p)$ em $p+\nu_{p} M$, definimos $\Delta_{p}^{+}(x)$ por

$$
\Delta_{p}^{+}(x)=\left\{v \in \Delta_{p}:\left\langle v, x-b_{p}\right\rangle>0\right\}
$$

Lema 2.3.9. O conjunto

$$
\Omega_{x}=\cap_{v \in \Delta_{p}^{+}(x)}\left\{y \in p+\nu_{p} M:\left\langle v, y-b_{p}\right\rangle>0\right\}
$$

é a componente conexa de $x$ em $F_{M}(p)^{c}$.

Demonstração. Com efeito, $\Omega_{x}$ é conexo pois é intersecção de convexos. De outro lado $\Omega_{x}$ é aberto e fechado em $F_{M}(p)^{c}$ e contém $x$.

Denotamos com $C_{x}$ o fecho de $\Omega_{x}$ em $p+\nu_{p} M$. A seguinte proposição diz que $C_{x}$ é um domínio fundamental de $W^{p}$, este é chamado câmera de Weyl de $W^{p}$ sobre $p+\nu_{p} M$.

Proposição 2.3.10. Dado $y \in p+\nu_{p} M$ a órbita de $y, W^{p}(y)$, dada pela ação de $W^{p}$ sobre $p+\nu_{p} M$, intersecta $C_{x}$ em um único ponto.

Demonstração. Consideremos $R \in \hat{W}^{p}$ tal que $\left\langle R\left(y-b_{p}\right), b_{p}-x\right\rangle$ seja maximal. Sejam $v \in \Delta_{p}^{+}(x)$ e 
$R_{v}$ a reflexão correspondente a $v$, então

$$
\begin{aligned}
\left\langle R\left(y-b_{p}\right), b_{p}-x\right\rangle & =\left\langle R_{v} R\left(y-b_{p}\right), b_{p}-x\right\rangle \\
& =\left\langle R\left(y-b_{p}\right), R_{v}\left(b_{p}-x\right)\right\rangle \\
& =\left\langle R\left(y-b_{p}\right), b_{p}-x-2\left\langle v, b_{p}-x\right\rangle v\right\rangle \\
& =\left\langle R\left(y-b_{p}\right), b_{p}-x\right\rangle+2\left\langle v, x-b_{p}\right\rangle\left\langle v, R\left(y-b_{p}\right)\right\rangle,
\end{aligned}
$$

$\operatorname{logo}\langle v, R(y-b p)\rangle \geq 0$, para todo $v \in \Delta_{p}^{+}(x)$. Seja $\rho \in W^{p}$ dado por $\rho(z)=R\left(z-b_{p}\right)+b_{p}$, $z \in p+\nu_{p} M$; segue que $\rho(y) \in C_{x}$. Para a demonstração da unicidade precisamos introduzir alguns objetos relativos à ação de $\hat{W}^{p}$ sobre $\nu_{p} M$ e considerar suas propriedades. Mas não queremos nos alongar muito nesta discussão. O leitor interessado pode consultar [Bro88] página 24 ou [BG70] capítulo 4 .

\subsection{Decomposição de subvariedades isoparamétricas do espaço Eu- clidiano}

Proposição 2.4.1. Seja $M$ uma subvariedade isoparamétrica completa de posto $l$ do espaço Euclidiano tal que suas normais de curvatura não se anulam. Então existem um ponto $p_{0}$ e um número real positivo $r_{0}$ tais que $M$ está contida na esfera de raio $r_{0}$ e centro em $p_{0}$

Demonstração. Pelo corolário (2.3.7) existe um campo normal paralelo $\zeta$ de $M$ tal que $\left\langle\zeta, \mathbf{n}_{i}\right\rangle=1$, para todo $i \in\{1, \ldots, g\}$. Seja $r_{0}=|\zeta|$. Consideremos a aplicação $\pi(p)=p+\zeta(p), p \in M$. Então para todo campo $X \in \Gamma(T M)$ e todo $p \in M$ temos que $d \pi_{p} X_{p}=X_{p}-A_{\zeta_{p}} X_{p}=0$. Assim $\pi$ é constante igual a $p_{0}$. Portanto para todo $p \in M$

$$
\left|p-p_{0}\right|=|\zeta(p)|=r_{0}
$$

Observação 2.4.2. Note que

$$
M_{\zeta}=\left\{p_{0}\right\}
$$


Também

$$
p_{0}=p+\zeta_{p}=b_{p} \in \cap_{i=1}^{g} l_{i}(p)
$$

para todo $p \in M$.

Corolário 2.4.3. Seja $M$ uma subvariedade isoparamétrica completa do espaço Euclidiano. Então as seguintes afirmações são equivalentes

a. $M$ é compacta

b. As normais de curvatura são não nulas

c. M está contida em uma esfera

Demonstração. Com efeito, se $M$ é compacta a observação (2.2.7) mostra que suas normais de curvatura são não nulas. Se as normais de curvatura de $M$ são não nulas a proposição anterior mostra que $M$ está contida em uma esfera. Se $M$ está contida em uma esfera, como $M$ é completa o teorema (2.6.2) implica que $M$ é fechada; assim $M$ é fechada em um compacto, portanto é compacta.

Teorema 2.4.4. Seja $M \subset \mathbf{E}^{n}$ uma subvariedade isoparamétrica completa e não compacta de posto l. Se $E_{0}$ é a distribuição de nulidade de $M$, então existe uma subvariedade isoparamétrica $N$, de posto $l$, de uma esfera de dimensão $n-m_{0}-1$ tal que

$$
M=N \times E_{0} .
$$

Demonstração. Seja $\mathbf{n}_{0}$ a normal de curvatura nula $\left(\mathbf{n}_{i}(p) \neq 0\right.$, para todo $i \in\{1, \ldots, g\}$ e todo $p \in M)$. Dado $p_{0} \in M$ fixo, consideremos o subespaço afim $\mathcal{P} \subset \nu_{p_{0}} M$ dado por $\mathcal{P}=\mathbf{n}_{1}\left(p_{0}\right)+$ $\sum_{i=2}^{g} \mathbf{R}\left(\mathbf{n}_{1}\left(p_{0}\right)-\mathbf{n}_{i}\left(p_{0}\right)\right)$. Temos que $\mathbf{n}_{0}\left(p_{0}\right)=0 \notin \mathcal{P}$, já que existe $v \in \nu_{p_{0}} M \operatorname{com}\left\langle\mathbf{n}_{i}\left(p_{0}\right), v\right\rangle=1$, para todo $i \in\{1, \ldots, g\}$. Consideremos a distribuição $\mathfrak{D}_{\mathcal{P}}=\oplus_{i=1}^{g} E_{i}$. Pela proposição $(2.2 .4) \mathfrak{D}_{\mathcal{P}}$ é auto-paralela e os operadores de forma de $M$ a preservam, então

$$
d U(X)=\nabla_{X} U \in \Gamma\left(E_{0}\right),
$$

para todo par de seções $U \in \Gamma\left(E_{0}\right)$ e $X \in \Gamma(T M)$. Portanto $E_{0}\left(p_{0}\right)=E_{0}(p)$ para todo $p \in M$. 
Seja $N=M \cap E_{0}^{\perp}$, vejamos que $N$ é uma subvariedade isoparamétrica do espaço Euclidiano $E_{0}^{\perp}$. Com efeito, $T_{p} N=\mathfrak{D}_{\mathcal{P}}(p)$ e $\nu_{p} N=\nu_{p} M$, para cada $p \in N$. Sejam $\hat{\nabla}^{\perp}$ e $\hat{A}$ a conexão normal e o operador de forma de $N$, respectivamente. Da fórmula Weingarten temos que

$$
\hat{A}_{\xi} X=\left.A_{\xi}\right|_{\mathfrak{D}_{\mathcal{P}}} X \text { e } \hat{\nabla}_{X}^{\perp} \xi=\nabla \frac{1}{X} \xi
$$

onde $X, Y \in \Gamma\left(\mathfrak{D}_{\mathcal{P}}\right)$ e $\xi \in \Gamma(\nu N)$. Portanto $N \subset E_{0}^{\perp}$ é isoparamétrica com normais de curvatura não nulas $\left.\mathbf{n}_{1}\right|_{N}, \ldots,\left.\mathbf{n}_{g}\right|_{N}$, logo $N$ tem posto $l$ e pela proposição (2.4.1) está contida em uma hiperesfera de $E_{0}^{\perp}$. Por último, se $p \in M, p=x_{0}+x \operatorname{com} x_{0} \in E_{0}$ e $x \in E_{0}^{\perp}, \operatorname{logo} x=p-x_{0} \in p+E_{0}=S_{0}(p) \subset M$, $\operatorname{assim} M \subset N \times E_{0}$. Agora, se $x \in N$ e $x_{0} \in E_{0}$ então $x+x_{0} \in x+E_{0}(x)=S_{0}(x) \subset M, \operatorname{logo}$ $N \times E_{0} \subset M$ e portanto $M=N \times E_{0}$.

Corolário 2.4.5. Seja $M \subset \mathbf{E}^{n}$ uma subvariedade isoparamétrica completa. Então $M$ é fechada.

Demonstração. Com efeito, se $M$ não tem normais de curvatura nulas então o corolário (2.4.3) implica que $M$ é compacta e portanto fechada. Agora, se $M$ tem uma normal de curvatura nula então o teorema (2.4.4) implica que $M$ é o produto de um fechado e um compacto, portanto é fechada.

Observação 2.4.6. Na proposição (2.2.4), supondo $M$ completa, a folha $S_{\mathcal{P}}(p)$ da distribuição $\mathfrak{D}_{\mathcal{P}}$ passando por $p$ também é completa, já que é totalmente geodésica em $M$. Assim pelo corolário acima é fechada em $L_{\mathcal{P}}(p)$, para todo $p \in M$.

Como conseqüência do corolário (2.2.6) e do teorema (2.4.4) temos a classificação das hipersuperfícies isoparamétricas do espaço Euclidiano.

Proposição 2.4.7. Seja $M$ uma hipersuperfície isoparamétrica conexa e completa do espaço Euclidiano. Então

a. Se $M$ tem uma única curvatura principal e é nula, então $M$ é um hiperplano.

b. Se $M$ tem uma única curvatura principal e é não nula, então $M$ é uma esfera.

c. Se $M$ tem duas curvaturas principais, então $M$ é um cilindro esférico.

Demonstração. Com efeito, $M$ tem posto um, logo 
a. $T M=E_{0}$, assim a afirmação segue do corolário (2.2.6).

b. $T M=E_{1}$, assim a afirmação segue do corolário (2.2.6).

c. $T M=E_{0} \oplus E_{1}$, assim o resultado segue do item b. e do teorema (2.4.4).

Teorema 2.4.8. Seja $M \subset \mathbf{E}^{n}$ uma subvariedade isoparamétrica conexa e completa com grupo de Coxeter $W$. Então $M$ é redutível se e somente se $W$ é redutível.

Demonstração. Suponhamos que existem dois espaços $V_{i} \subset \mathbf{E}^{n}$ e duas subvariedades isoparamétricas e substanciais $M_{i} \subset p_{0}+V_{i}$, para $i=1,2$, com $M=M_{1} \times M_{2}$ e $V_{1} \perp V_{2}$. Portanto $\nu_{p_{1}} M_{1} \perp \nu_{p_{2}} M_{2}$, para todo $p_{i}, \in M_{i}, i=1,2$. Assim o grupo de Coxeter $\mathrm{W}$ de $M$ é o produto direto $W=W_{1} \times W_{2}$, onde $W_{i}$ é o grupo de Coxeter de $M_{i}$, para $i=1,2$.

Reciprocamente suponhamos que $W=W_{1} \times W_{2}$. Pelo teorema anterior podemos supor $\mathbf{n}_{i} \neq 0$, para todo $i \in\{1, \ldots, g\}$. Seja $p_{0} \in M$ fixo. Então existem dois subespaços $\mathcal{P}_{1}$ e $\mathcal{P}_{2}$ de $\nu_{p_{0}} M$ com $\mathcal{P}_{1} \perp \mathcal{P}_{2}, \nu_{p_{0}} M=\mathcal{P}_{1} \oplus \mathcal{P}_{2}$ e as normais de curvatura que geram $W_{i}$ pertencem a $\mathcal{P}_{i}$, para $i=1,2$. Consideremos a notação da proposição (2.2.4) correspondente a $\mathcal{P}_{1}$ e $\mathcal{P}_{2}$. Dadas seções $X_{i}+\xi_{i}$ de $\mathbf{F}_{\mathcal{P}_{i}}$ e $Y_{j}$ de $\mathfrak{D}_{\mathcal{P}_{j}}$, com $i \neq j$ temos que

$$
d\left(X_{i}+\xi_{j}\right)\left(Y_{j}\right)=\nabla_{Y_{j}} X_{i}+\nabla_{Y_{j}}^{\perp} \xi_{i} \in \Gamma\left(\mathbf{F}_{\mathcal{P}_{i}}\right)
$$

Logo, dado $p \in M$ temos que

$$
\mathbf{F}_{\mathcal{P}_{i}}(q)=\mathbf{F}_{\mathcal{P}_{i}}(p),
$$

para todo $q \in S_{\mathcal{P}_{j}(p)}$, com $i, j=1,2$ (o caso $i=j$ segue da equação (2.10)).

Dado $p \in M$, sejam $x \in S_{\mathcal{P}_{1}}(p)$ e $y \in S_{\mathcal{P}_{2}}(p)$. Então

$$
S_{\mathcal{P}_{1}}(y) \cap S_{\mathcal{P}_{2}}(x)=\{x+y-p\} .
$$

Com efeito. Pela observação (2.4.6) as folhas das distribuições $D_{\mathcal{P}_{i}}, i=1,2$, são completas. Seja $\beta:[0,1] \longrightarrow S_{\mathcal{P}_{2}}(p)$ uma geodésica, $\operatorname{com} \beta(0)=p, \beta(1)=y$ e $\beta^{\prime}(0)=u$. Sejam $\gamma:[0,1] \longrightarrow S_{\mathcal{P}_{1}}(p)$ uma curva suave por partes, com $\gamma(0)=p$ e $\gamma(1)=x$, e $U(t)$ o transporte paralelo de $u$ ao longo de $\gamma$. Consideremos a família a um parâmetro de geodésicas $\beta_{s}(t)=\exp _{\gamma(s)}(t U(s))$, onde exp é a aplicação 
exponencial de $M$. Sejam $J(t)=\left.\frac{\nabla}{d s}\right|_{s=0} \beta_{s}(t)$ e $J_{i}(t)$ sua projeção ortogonal sobre $\mathfrak{D}_{\mathcal{P}_{i}}(\beta(t))$, para $i=1,2$ e cada $t \in[0,1]$. Temos que

$$
J(0)=\left.\frac{\nabla}{d s}\right|_{s=0} \beta_{s}(0)=\left.\frac{\nabla}{d s}\right|_{s=0} \gamma(s)=\gamma^{\prime}(0) \in \mathfrak{D}_{\mathcal{P}_{1}}(p)
$$

e

$$
\begin{aligned}
J^{\prime}(0) & =\left.\left.\frac{\nabla}{d t}\right|_{t=0} \frac{\nabla}{d s}\right|_{s=0} \beta_{s}(t) \\
& =\left.\left.\frac{\nabla}{d s}\right|_{s=0} \frac{\nabla}{d t}\right|_{t=0} \beta_{s}(t) \\
& =\left.\frac{\nabla}{d s}\right|_{s=0} \beta_{s}^{\prime}(0) \\
& =\left.\frac{\nabla}{d s}\right|_{s=0} U(s) \\
& =0 .
\end{aligned}
$$

Assim $J_{2}(0)=J_{2}^{\prime}(0)=0$. Como $J$ é um campo de Jacobi e $\mathfrak{D}_{\mathcal{P}_{2}}$ é auto-paralela então $J_{2}$ é um campo de Jacobi, $\operatorname{logo} J_{2}=0$. Portanto

$$
\frac{\nabla}{d s} \beta_{s}(t) \in \mathfrak{D}_{\mathcal{P}_{1}}(\beta(t)),
$$

para todo $t \in[0,1]$. Como $u \in \mathfrak{D}_{\mathcal{P}_{2}}(p)$, então $U(s) \in \mathfrak{D}_{\mathcal{P}_{2}}(\gamma(s))$ e como $S_{\mathcal{P}_{2}}(\gamma(s)) \subset M$ é totalmente geodésica tem-se $\beta_{s}(t) \in S_{\mathcal{P}_{2}}(\gamma(s))$. Em particular $\beta_{1}(1) \in S_{\mathcal{P}_{2}}(x)$. Por $(2.21) \frac{\nabla}{d s} \beta_{s}(1) \in \mathfrak{D}_{\mathcal{P}_{1}}(\beta(1))$, então a curva $s \mapsto \beta_{s}(1)$ está contida em $S_{\mathcal{P}_{1}}(y)$. Logo

$$
\beta_{1}(1) \in S_{\mathcal{P}_{1}}(y) \cap S_{\mathcal{P}_{2}}(x) .
$$

De outro lado temos que $y-p \in \mathbf{F}_{\mathcal{P}_{2}}(p), x-p \in \mathbf{F}_{\mathcal{P}_{1}}(p)$ e $\mathbf{F}_{\mathcal{P}_{1}}(p) \cap \mathbf{F}_{\mathcal{P}_{2}}(p)=\{0\}$. Então a equação (2.19) implica que

$$
\begin{aligned}
L_{\mathcal{P}_{1}}(y) \cap L_{\mathcal{P}_{2}}(x) & =\left(y+\mathbf{F}_{\mathcal{P}_{1}}(y)\right) \cap\left(x+\mathbf{F}_{\mathcal{P}_{2}}(x)\right) \\
& =\left(x+y-p+\mathbf{F}_{\mathcal{P}_{1}}(p)\right) \cap\left(x+y-p+\mathbf{F}_{\mathcal{P}_{2}}(p)\right) \\
& =\{x+y-p\} .
\end{aligned}
$$

Assim $\emptyset \neq S_{\mathcal{P}_{1}}(y) \cap S_{\mathcal{P}_{2}}(x) \subset L_{\mathcal{P}_{1}}(y) \cap L_{\mathcal{P}_{2}}(x)=\{x+y-p\}$. Isto prova $(2.20)$. 
Dado $p \in M$, para $x \in S_{\mathcal{P}_{1}}(p)$, consideremos a aplicação $f_{x}(y)=y+(x-p), y \in \mathbf{E}^{n}$. Por $(2.20) f_{x}\left(S_{\mathcal{P}_{2}}(p)\right) \subset S_{\mathcal{P}_{2}}(x)$ e $f_{x}^{-1}\left(S_{\mathcal{P}_{2}}(x)\right) \subset S_{\mathcal{P}_{2}}(p)$. Como $f_{x}$ é uma isometria de $\mathbf{E}^{n}$, leva $S_{\mathcal{P}_{2}}(p)$ isometricamente sobre $S_{\mathcal{P}_{2}}(x)$.

Por último seja $\phi: L_{\mathcal{P}_{1}}(p) \times L_{\mathcal{P}_{2}}(p) \longrightarrow \mathbf{E}^{n}$, dada por $\phi(x, y)=x+y-p$. Então como $\mathbf{F}_{\mathcal{P}_{2}}=\mathbf{F}_{\mathcal{P}_{1}}^{\perp}$, $\phi$ é uma isometria. Na outra mão, da observação (2.4.6) $S_{\mathcal{P}_{i}}(p)$ é uma subvariedade fechada de $L_{\mathcal{P}_{i}}(p)$, para $i=1,2, \operatorname{logo} S_{\mathcal{P}_{1}}(p) \times S_{\mathcal{P}_{2}}(p) \subset L_{\mathcal{P}_{1}}(p) \times L_{\mathcal{P}_{2}}(p)$ é fechada. Se $(x, y) \in S_{\mathcal{P}_{1}}(p) \times S_{\mathcal{P}_{2}}(p)$, então $\phi(x, y)=f_{x}(y) \in S_{\mathcal{P}_{2}}(x)$. Assim $\phi\left(S_{\mathcal{P}_{1}}(p) \times S_{\mathcal{P}_{2}}(p)\right) \subset M$ é uma subvariedade aberta e fechada de $M$ logo coincide com M. Da proposição (2.2.4) $S_{\mathcal{P}_{i}}(p)$ é uma subvariedade isoparamétrica e substancial de $L_{\mathcal{P}_{i}}(p)$, para $i=1,2$. Isto completa a prova.

\subsection{Teorema da fatia ${ }^{\dagger}$}

Lema 2.5.1. Seja $M$ uma subvariedade isoparamétrica do espaço Euclidiano. Dado um campo normal paralelo consideremos a variedade focal $\pi: M \longrightarrow M_{\xi}$. Dado $q \in M_{\xi}$, seja $F_{q}$ uma componente conexa da fibra $\pi^{-1}(q)$. Então $F_{q}$ é uma subvariedade isoparamétrica de $q+\nu_{q} M_{\xi}$.

Demonstração. Seja $q \in M_{\xi}$ fixo. Temos que para cada $p \in \pi^{-1}(q)$

$$
\operatorname{ker} d \pi_{p}=\bigoplus_{q \in l_{i}(p)} E_{i}(p)
$$

Do lema (2.1.2) os operadores de forma de $M$ preservam a decomposição ortogonal $T M=\operatorname{ker} d \pi \oplus$ ker $d \pi^{\perp}$ e as componentes conexas das fibras de $\pi$ são subvariedades totalmente geodésicas de $M$. Então, por um razonamento análogo ao feito na demonstração do item c. da proposição $(2.2 .4), F_{q}$ é uma subvariedade isoparamétrica de

$$
p+\bigoplus_{q \in l_{i}(p)} E_{i}(p) \oplus \nu_{p} M=p+\nu_{q} M_{\xi}=q+\nu_{q} M_{\xi}
$$

\footnotetext{
†Do inglês 'slice'.
} 
Observação 2.5.2. A existência de polinômios isoparamétricos (vide seção 2.6) implicará que as variedades paralelas e focais de uma variedade isoparamétrica $M \subset \mathbf{E}^{n}$ constituem uma folheação singular de $\mathbf{E}^{n}$. Nós não provaremos este fato. Para uma demonstração vide [PT88] capitulo 6 .

Teorema 2.5.3 (Teorema da fatia). Seja $M_{\xi}$ uma variedade focal de uma subvariedade isoparamétrica $M \subset \mathbf{E}^{n}$. Se $q \in M_{\xi}$ é fixo então as variedades paralelas $M_{\eta}$ de $M$ intesectam $q+\nu_{q} M_{\xi}$ em uma folheação isoparamétrica de $q+\nu_{q} M_{\xi}$.

Demonstração. Seja $\hat{M}=M_{\eta}$ definimos $\zeta_{\hat{p}}=\xi_{p}-\eta_{p}$, onde $\hat{p}=p+\eta_{p}$. Consideremos a projeção $\hat{\pi}: \hat{M} \longrightarrow \hat{M}_{\zeta}$. Pela fórmula do tubo temos que

$$
d \hat{\pi}_{\hat{p}}=i d_{T_{p} M}-A_{\xi_{p}-\eta_{p}}\left(i d_{T_{p} M}-A_{\eta_{p}}\right)^{-1},
$$

$\operatorname{logo} \operatorname{ker} d \hat{\pi}_{\hat{p}}=\left(i d_{T_{p} M}-A_{\eta_{p}}\right) \operatorname{ker} d \pi_{p}$. Então $\hat{M}_{\zeta}=M_{\xi}$ é uma variedade focal de $\hat{M}$. Se $\phi(p)=p+\eta_{p}$, $p \in M$, então $\phi$ é um difeomorfismo de $M$ sobre $\hat{M}$. Fixando $q \in M_{\xi}$, temos que

$$
\hat{\pi}^{-1}(q)=\phi\left(\pi^{-1}(q)\right)
$$

Assim as componentes conexas $\hat{F}_{q}$ de $\hat{\pi}^{-1}(q)$ são da forma $\phi\left(F_{q}\right)$, onde $F_{q}$ é uma componente conexa de $\pi^{-1}(q)$. Isto é $\hat{F}_{q}$ é uma variedade paralela de $F_{q}$. Do lema anterior $\hat{F}_{q}$ é uma subvariedade isoparamétrica de $q+\nu_{q} \hat{M}_{\zeta}=q+\nu_{q} M_{\xi}$.

Lema 2.5.4. Seja $M$ uma subvariedade isoparamétrica do espaço Euclidiano. Dado um campo normal paralelo consideremos a variedade focal ou paralela $\pi: M \longrightarrow M_{\xi}$. Então para $q \in M_{\xi}$ tem-se

$$
\nu_{q} M_{\xi}=\cup_{p \in \pi^{-1}(q)} \nu_{p} M
$$

Demonstração. Sabemos que para cada $p \in \pi^{-1}(q), \nu_{p} M \subset \nu_{q} M_{\xi}$. Agora, dado $v \in \nu_{q} M_{\xi}$ existe $\eta \in \Gamma(\nu M)$ tal que $q+v \in M_{\eta} \cap \pi^{-1}(q)$, ou seja $v=\eta_{p}-\xi_{p} \in \nu_{p} M$, para algum $p \in \pi^{-1}(q)$.

\subsection{Aplicações isoparamétricas}

Seja $N$ uma forma espacial. Dizemos que $f=\left(f_{1}, \ldots, f_{n}\right): N \longrightarrow \mathbf{R}^{k}$ é uma aplicação isoparamétrica se 
AI 1. $f$ tem um valor regular;

AI 2. $\Delta f_{i}$ e $\left\langle\operatorname{grad} f_{i}, \operatorname{grad} f_{j}\right\rangle$ são funções de $f$;

AI 3. $\left[\operatorname{grad} f_{i}, \operatorname{grad} f_{j}\right]$ é uma combinação linear de $\operatorname{grad} f_{1}, \ldots, \operatorname{grad} f_{k}$, tal que os coeficientes são funções de $f$,

para todo par $i, j \in\{1, \ldots, k\}$, onde $\Delta$ e grad denotam o Laplaciano e o gradiente, respectivamente.

Teorema 2.6.1. Uma subvariedade $M \subset \mathbf{E}^{n}$ é isoparamétrica se e somente se é, localmente, um conjunto de nível regular de uma aplicação isoparamétrica.

Demonstração. Suponhamos que $f: \mathcal{A} \longrightarrow \mathbf{R}^{k}$ é uma aplicação isoparamétrica onde $\mathcal{A} \subset \mathbf{E}^{n}$ é um aberto. Seja $c$ um valor regular de $f$, o qual existe por AI 1. Diminuindo $\mathcal{A}$, se necessário, $\mathcal{B}=f(\mathcal{A})$ é uma vizinhança aberta de $c$ em $\mathbf{R}^{k}$ tal que $f: \mathcal{A} \longrightarrow \mathcal{B}$ é uma submersão. Equiparemos $\mathcal{B}$ com uma métrica tal que $f$ seja uma submersão Riemanniana de $\mathcal{A}$ sobre $\mathcal{B}$. Com efeito, sejam $a_{i j}=\left\langle\operatorname{grad} f_{i}, \operatorname{grad} f_{j}\right\rangle$, para todo par $i, j \in\{1, \ldots, k\}$. Por AI 2 , as funções $a_{i j}$ são constantes ao longo das fibras de $f$, portanto podemos considerar-las como funções definidas em $\mathcal{B}$. Assim, dado $q \in \mathcal{B}$ temos que

$$
d f_{p} \operatorname{grad} f_{i}(p)=\left(a_{1 i}(q), \ldots, a_{n i}(q)\right),
$$

para todo $p \in f^{-1}(q)$. Seja $w \in T_{q} \mathcal{B}$, então para cada $p \in f^{-1}(q)$ existe $u(p)=\sum_{i=1}^{k} c_{i}(p) \operatorname{grad} f_{i}(p)$ tal que

$$
w=d f_{p} u(p) .
$$

Se $p, p^{\prime} \in f^{-1}(q)$ temos que

$$
\begin{aligned}
\sum_{i=1}^{k} c_{i}(p)\left(a_{1 i}(q), \ldots, a_{n i}(q)\right) & =d f_{p} u(p) \\
& =w \\
& =d f_{p^{\prime}} u\left(p^{\prime}\right) \\
& =\sum_{i=1}^{k} c_{i}\left(p^{\prime}\right)\left(a_{1 i}(q), \ldots, a_{n i}(q)\right) .
\end{aligned}
$$

Portanto os coeficientes $c_{1}, \ldots, c_{k}$ independem de $p \in f^{-1}(q)$. Desta maneira podemos definir a 
métrica $\langle\langle\cdot, \cdot\rangle\rangle$ sobre $\mathcal{B}$ por

$$
\left\langle\left\langle w, w^{\prime}\right\rangle\right\rangle_{q}=\sum_{i, j=1}^{k} c_{i} c_{j}^{\prime} a_{i j}(q),
$$

para todo par de vetores $w, w^{\prime} \in T_{q} \mathcal{B}$ e todo $q \in \mathcal{B}$. Com esta métrica $f: \mathcal{A} \longrightarrow \mathcal{B}$ é uma submersão Riemanniana ${ }^{\ddagger}$.

Por AI 3 a distribuição horizontal de $\mathcal{A}$ em relação a $f$ é integrável, logo a proposição (1.6.1) implica que é auto-paralela. A equação $(2.22)$ diz que os campos horizontais $\operatorname{grad} f_{1}, \ldots, \operatorname{grad} f_{k}$ são projetáveis (logo são básicos). Assim, de novo, a proposição (1.6.1) implica que estes campos são paralelos em relação às conexões normais das fibras de $f$ e como constituem um referencial normal global, estas têm fibrado normal raso.

Seja $\mathbf{e}_{1}, \ldots, \mathbf{e}_{n-k}, \varepsilon_{1}, \ldots \varepsilon_{k}$ um referencial ortonormal local de $\mathcal{A}$ tal que $\mathbf{e}_{1}, \ldots, \mathbf{e}_{n-k}$ e $\varepsilon_{1}, \ldots \varepsilon_{k}$ são referenciais das distribuições vertical e horizontal dada por $f$, respectivamente, onde o segundo está constituído por campos básicos. Denotemos por $\bar{\nabla}$ e $[\cdot, \cdot]$ a conexão de Levi-Civita e o colchete de Lie de $\mathbf{E}^{n}$, respectivamente. Sejam $i, j, l \in\{1, \ldots, k\}$ e $\beta \in\{1, \ldots, n-k\}$ fixos. Sabemos que $\left[\mathbf{e}_{\beta}, \varepsilon_{l}\right]$ é uma seção vertical e distribuição horizontal é auto-paralela, assim $\left\langle\bar{\nabla}_{\varepsilon_{j}} g r a d f_{i},\left[\mathbf{e}_{\beta}, \varepsilon_{l}\right]\right\rangle=0$ e portanto

$$
\begin{aligned}
\left\langle\bar{\nabla}_{\varepsilon_{j}} g r a d f_{i}, \bar{\nabla}_{\left.\mathbf{e}_{\beta} \varepsilon_{l}\right\rangle}\right\rangle & =\left\langle\bar{\nabla}_{\varepsilon_{j}} g r a d f_{i}, \bar{\nabla}_{\varepsilon_{l}} \mathbf{e}_{\beta}\right\rangle \\
& =\varepsilon_{l}\left\langle\bar{\nabla}_{\varepsilon_{j}} \operatorname{grad} f_{i}, \mathbf{e}_{\beta}\right\rangle-\left\langle\bar{\nabla}_{\varepsilon_{l}} \bar{\nabla}_{\varepsilon_{j}} \operatorname{grad} f_{i}, \mathbf{e}_{\beta}\right\rangle \\
& =0 .
\end{aligned}
$$

Agora, como $\mathbf{E}^{n}$ tem tensor de curvatura nulo, $\operatorname{grad} f_{i}$ é paralelo e $\left[\mathbf{e}_{\beta}, \varepsilon_{l}\right]$ é vertical, da equação acima tem-se

$$
\begin{aligned}
\mathbf{e}_{\beta}\left\langle\bar{\nabla}_{\varepsilon_{j}} g r a d f_{i}, \varepsilon_{l}\right\rangle & =\left\langle\bar{\nabla}_{\mathbf{e}_{\beta}} \bar{\nabla}_{\varepsilon_{j}} \operatorname{grad} f_{i}, \varepsilon_{l}\right\rangle+\left\langle\bar{\nabla}_{\varepsilon_{j}} \operatorname{grad} f_{i}, \bar{\nabla}_{\left.\mathbf{e}_{\beta} \varepsilon_{l}\right\rangle}\right\rangle \\
& =\left\langle\bar{\nabla}_{\mathbf{e}_{\beta}} \bar{\nabla}_{\varepsilon_{j}} \operatorname{grad} f_{i}, \varepsilon_{l}\right\rangle \\
& =\left\langle\left(\bar{\nabla}_{\varepsilon_{j}} \bar{\nabla}_{\mathbf{e}_{\beta}}+\bar{\nabla}_{\left[\mathbf{e}_{\beta}, \varepsilon_{j}\right]}\right) \operatorname{grad} f_{i}, \varepsilon_{l}\right\rangle \\
& =\left\langle\bar{\nabla}_{\varepsilon_{j}} \bar{\nabla}_{\mathbf{e}_{\beta}} \operatorname{grad} f_{i}, \varepsilon_{l}\right\rangle \\
& =0 .
\end{aligned}
$$

\footnotetext{
${ }^{\ddagger}$ Note que a matriz de $\langle\langle\cdot, \cdot\rangle\rangle$ nas coordenadas usuais de $\mathbf{R}^{k}$ é $\left[a_{i j}\right]^{-1}$.
} 
Logo $\left\langle\bar{\nabla}_{\varepsilon_{j}} g r a d f_{i}, \varepsilon_{l}\right\rangle$ é constante ao longo das fibras de $f$, para toda trio $i, j, l \in\{1, \ldots, k\}$.

Consideremos um conjunto de nível regular $M$ de $f$. Sejam $\nabla, H$ e $A$ sua conexão de Levi-Civita e seus campo de curvatura média e operador de forma, respectivamente. Temos que

$$
\left.\operatorname{grad} f_{1}\right|_{M}, \ldots,\left.\operatorname{grad} f_{k}\right|_{M}
$$

é um referencial paralelo de $\nu M$. Se $i \in\{1, \ldots, k\}$ tem-se

$$
\begin{aligned}
(n-k)\left\langle H, \operatorname{grad} f_{i}\right\rangle & =-\sum_{j=1}^{n-k}\left\langle\bar{\nabla}_{\mathbf{e}_{j}} \operatorname{grad} f_{i}, \mathbf{e}_{j}\right\rangle \\
& =\sum_{j=1}^{k}\left\langle\bar{\nabla}_{\varepsilon_{j}} \operatorname{grad} f_{i}, \varepsilon_{j}\right\rangle-\Delta f_{i} .
\end{aligned}
$$

Então $\left\langle H, \operatorname{grad} f_{i}\right\rangle$ é constante e portanto $\nabla^{\perp} H=0$. Seja $X$ um campo básico, então $\xi=\left.X\right|_{M} \in \nu M$. Denotando com exp e $e^{\prime} p^{\prime}$ as aplicações exponenciais do espaço Euclidiano e de $\mathcal{B}$, respectivamente. Como $f$ e $d f(\xi)$ são constantes ao longo de $\mathrm{M}$ e $f$ é uma isometria existe $q_{0} \in \mathcal{B}$ tal que

$$
f\left(p+\xi_{p}\right)=f\left(\exp _{p}\left(\xi_{p}\right)\right)=\exp _{f(p)}^{\prime}\left(d f_{p} \xi_{p}\right)=q_{0},
$$

para todo $p \in M$. Portanto $M_{\xi} \subset f^{-1}\left(q_{0}\right)$. Escolhendo $|\xi|<t_{0}$, com $t_{0}$ suficientemente pequeno para que $M_{\xi}$ seja uma variedade paralela de $M$ e $q_{0}$ seja um valor regular de $f$ temos que $\operatorname{dim} M_{\xi}=\operatorname{dim} M$, e portanto $M_{\xi}$ é um aberto de $f^{-1}\left(q_{0}\right)$. Agora, sejam $\mathcal{C} \subset M$ uma bola fechada de raio positivo e $R=\max \left\{\left|A_{\xi_{p}}\right|: p \in \mathcal{C}\right\}^{\S}$. Denotemos com $A(s)$ e $H(s)$ o operador de forma e campo de curvatura média de $M_{s \xi}$, para $s \in\left(0, t_{1}\right)$ onde $\left.t_{1}=\min \left\{1, \frac{1}{R}\right)\right\}$. Temos que $H(s)$ é constante (já que $M_{s \xi}$ é um aberto de um conjunto de nível regular de $f$ ) e pela fórmula do tubo tem-se

$$
A(s)_{\xi}=A_{\xi}\left(i d-A_{s \xi}\right)^{-1}=A_{\xi} \sum_{i=0}^{\infty}\left(A_{s \xi}\right)^{i}=\sum_{i=0}^{\infty} s^{i}\left(A_{\xi}\right)^{i+1},
$$

\footnotetext{
${ }^{\S} \mathrm{A}$ aplicação $p \mapsto\left|A_{\xi_{p}}\right|=\max _{|u|=1}\left\{\left|A_{\xi_{p}} u\right|\right\}=\max \left\{\left|\lambda_{1}\left(\xi_{p}\right)\right|, \ldots,\left|\lambda_{g}\left(\xi_{p}\right)\right|\right\}, p \in M$, é contínua. Logo tem um ponto de máximo no compacto $\mathcal{C}$. Por outro lado, lembre que se $A$ é um operador linear contínuo de um espaço de Banach tal que $|A|<1$, então $(i d-A)^{-1}=\sum_{i=0}^{\infty} A^{i}$.
} 
$\log 0$

$$
(n-k)\langle H(s), \xi\rangle=\operatorname{traço}\left(A(s)_{\xi}\right)=\sum_{i=0}^{\infty} \operatorname{traço}\left(\left(A_{\xi}\right)^{i+1}\right) s^{i} .
$$

O membro esquerdo da equação acima independe de $p \in \mathcal{C}$, logo os coeficientes da série de potências do membro direito da mesma são constantes em $\mathcal{C}$. Segue que $M$ tem curvaturas principais constantes ao longo de $\left.\xi\right|_{\mathcal{C}}$. Por último, se dois pontos em $M$ estão unidos por uma curva, existe uma bola fechada de raio positivo que contem essa curva e está contida em $M$. Logo $M$ tem curvaturas principais constantes ao longo de campos normais paralelos ao longo de curvas.

A prova da necessidade é um pouco técnica e na verdade e possível mostrar que variedades isoparamétricas são, globalmente, conjuntos de nível regulares de polinômios isoparamétricos (ou seja aplicações isoparamétricas polinomiais). A saber, temos o seguinte resultado.

Teorema 2.6.2. Seja $M \subset \mathbf{E}^{m+l}$ uma subvariedade isoparamétrica de dimensão $m$; completa, de posto $l$ e com normais de curvatura não nulas. Dado $p \in M$ fixo, sejam $h_{1}, \ldots, h_{l}$ os geradores do anel de polinômios $W^{p}$-invariantes sobre $p+\nu_{p} M$. Então $h=\left(h_{1}, \ldots, h_{l}\right)$ tem uma única extensão a um polinômio isoparamétrico $f: \mathbf{E}^{m+l} \longrightarrow \mathbf{R}^{l}$, tal que $M=f^{-1}(f(p))$, onde $f(p)$ é um valor regular de $f$.

O leitor interessado poderá encontrar a prova em [PT88] (seção 6.4).

Corolário 2.6.3. Tem-se

a. A intersecção $M \cap\left(p+\nu_{p} M\right)$ é a órbita $W^{p}(p)$ da ação de $W^{p}$ sobre $p+\nu_{p} M$.

b. M é mergulhada.

c. $\nu M$ é globalmente raso, isto é, existe um referencial normal paralelo definido sobre $M$.

Demonstração. Com efeito,

a. Como $h$ é $W^{p}$-invariante e $f$ estende a $h$, então $h(q)=f(q)=f(p)$, ou seja $q \in f^{-1}(f(p))$, para todo $q \in W^{p}(p)$. Segue que $W^{p}(p) \subset M \cap\left(p+\nu_{p} M\right)$. Agora, o polinômio $h$ separa as órbitas de $W_{p}$, então $M$ não contem elementos de outras órbitas diferentes a $W^{p}(p), \log 0 M \cap\left(p+\nu_{p} M\right)=W^{p}(p)$.

b. $M$ é um conjunto de nível regular de $f$. 
c. Seja $\mathcal{B}=f\left(\mathbf{E}^{m+l}\right)$. Procedendo de maneira análoga como na demonstração do teorema (2.6.1) dotamos $\mathcal{B}$ de uma métrica que faz com que $f: \mathbf{E}^{m+l} \longrightarrow \mathcal{B}$ seja uma submersão Riemanniana. Como $f$ é uma aplicação isoparamétrica concluímos que $\operatorname{grad} f_{1}, \ldots, \operatorname{grad} f_{l}$ é um referencial normal paralelo definido em $M$.

\subsection{Notas adicionais}

O problema da classificação tem um papel principal no estudo de qualquer objeto matemático. No nosso caso já o Levi-Civita classificou as famílias de superfícies isoparamétricas do espaço Euclidiano tridimensional $\mathbf{E}^{3}$ como sendo planos paralelos, esferas concêntricas e cilindros coaxiais esféricos. Segre generalizou este resultado para hipersuperfícies do espaço Euclidiano $\mathbf{E}^{n} \operatorname{com} n$ arbitrário. Esta é a proposição (2.4.7). Da mesma maneira Cartan mostrou o resultado análogo para o caso de hipersuperfícies isoparamétricas do espaço hiperbólico $\mathbf{H}^{n}$. Portanto as hipersuperfícies isoparamétricas de $\mathbf{E}^{n}$ e $\mathbf{H}^{n}$ estão classificadas.

Agora, para o caso da esfera $\mathbf{S}^{n}$ o problema da classificação das hipersuperfícies isoparamétricas ainda está em aberto. Mas tem-se avançado muito e o problema está na última etapa. Os primeiros passos neste sentido foram dados por Cartan e mais adiante por Münzner, entre outros. O principal resultado deste último foi a determinação do número $g$ de curvaturas principais de uma hipersuperfície isoparamétrica de $\mathbf{S}^{n}$, a saber $g \in\{1,2,3,4,6\}$. Cartan resolveu o problema para $g \leq 3$. Para $g=1,2$ as hipersuperfícies são esferas no primeiro caso e produto de esferas no segundo. Para $g=3$ Cartan mostrou que as curvaturas principais têm a mesma multiplicidade $m \in\{1,2,4,8\}$ e as hipersuperfícies isoparamétricas são tubos de raio constante sobre mergulhos de Veronese do plano projetivo $\mathbf{F P}^{2}$ em $\mathbf{S}^{3 m+1}$ onde $\mathbf{F}$ é a álgebra de divisão $\mathbf{R}, \mathbf{C}, \mathbf{H}$ (quatérnios) e $\mathbf{O}$ (números de Cayley) para $m=1,2,4,8$, respectivamente.

Münzner provou que se $g=6$ as curvaturas principais têm as mesmas multiplicidades $m$. Abresch mostrou que $m \in\{1,2\}$. Em 1985 Dorfmeister e Neher mostraram que para $m=1$ as hipersuperfícies isoparamétricas são homogêneas. Para o caso $m=2$ o problema da classificação está em aberto. Ademais existem dois exemplos de famílias de hipersuperfícies isoparamétricas homogêneas com seis curvaturas principais. Uma em $\mathbf{S}^{7}$ com multiplicidades iguais a um e outra em $\mathbf{S}^{13}$ com multiplicidades iguais a dois. 
Existem bastantes e diversos exemplos de hipersuperfícies isoparamétricas da esfera com $g=4$ curvaturas principais. Cartan construiu exemplos em $\mathbf{S}^{5}$ e $\mathbf{S}^{9}$. Esses exemplos são homogêneos e têm as multiplicidades iguais. Ele formulou duas perguntas: São todas as hipersuperfícies isoparamétricas homogêneas? Existe alguma hipersuperfície isoparamétrica cujas multiplicidades das curvaturas principais sejam distintas? Nomizu respondeu a segunda pergunta afirmativamente; a saber, encontrou uma família de hipersuperfícies isoparamétricas com multiplicidades $m_{1}=1$ e $m_{2}=k$, para um inteiro positivo arbitrário $k$. Takagi e Takahashi mostraram que toda hipersuperfície isoparamétrica homogênea da esfera é uma órbita principal de uma representação isotrópica de um espaço simétrico de posto dois. Além disso incluíram uma ampla lista de exemplos. Takagi demonstrou que para $g=4$, se uma das curvaturas principais tem multiplicidade igual a um, então a hipersuperfície isoparamétrica é homogênea. Ozeki e Takahashi produziram duas famílias infinitas de hipersuperfícies isoparamétricas não homogêneas com multiplicidades $m_{1}=3, m_{2}=4 k$ e $m_{1}=7, m_{2}=8 k$, onde $k$ é um inteiro positivo arbitrário. Eles também demonstraram que toda hipersuperfície isoparamétrica da esfera, tal que uma das curvaturas principais tem multiplicidade igual a um, é homogênea. Para uma discussão mais ampla deste assunto vide [Tho00], [CCJ07] e [PT88] capítulo 5.

Como vimos no começo do capítulo 2 , subvariedades isoparamétricas de $\mathbf{S}^{n}$ são também subvariedades isoparamétricas de $\mathbf{E}^{n+1}$ e vice-versa. Logo classificar as hipersuperfícies da esfera é equivalente a classificar subvariedades isoparamétricas de posto dois (com normais de curvatura não nulas) do espaço Euclidiano. Para o caso de posto maior o igual a três Thorbergsson separadamente de Olmos e usando diferentes técnicas, mostraram que subvariedades isoparamétricas substanciais e irredutíveis são órbitas de um subgrupo de isometrias do espaço Euclidiano. Heintze e Liu generalizaram este resultado para o caso de dimensão infinita em [HL99]. 
CAPÍTULO 3

Exemplos

Neste capítulo daremos alguns exemplos de subvariedades isoparamétricas. Basicamente encontraremos as curvaturas principais de uma hipersuperfície da esfera e daremos exemplos de subvariedades isoparamétricas homogêneas dadas pela ação adjunta de um grupo de Lie compacto.

\subsection{Hipersuperfícies da esfera $\mathrm{S}^{n}$}

Seja $M \subset \mathbf{S}^{n}$ uma hipersuperfície isoparamétrica. Sabemos que um campo normal unitário $\zeta$ de $M$ em relação a $\mathbf{E}^{n+1}$ é da forma

$$
\zeta=\cos t \xi-\sin t P
$$

onde $\xi$ é um campo normal unitário de $M$ em relação a $\mathbf{S}^{n}$ e $P$ é o campo vetor posição de $\mathbf{E}^{n+1}$ restrito a $\mathbf{S}^{n}$. Sejam $\lambda_{1}, \ldots, \lambda_{g}$ as curvaturas principais de $M$ em $\mathbf{S}^{n}$ então as curvaturas principais de $M$ em $\mathbf{E}^{n+1}$ são da forma

$$
\lambda_{i} \cos t+\sin t
$$


e portanto

$$
\mathbf{n}_{i}=\lambda_{i} \xi-P,
$$

para todo $i \in\{1, \ldots, g\}$. Denotemos com $\theta_{i}$ o ângulo entre $\mathbf{n}_{i}$ e $\xi$ dado pela orientação $\left\{\xi_{p},-p\right\}$ do espaço normal a $M$ em $p$. Então

$$
\lambda_{i}=\cot \theta_{i},
$$

para todo $i \in\{1, \ldots, g\}$. Sem perder generalidade podemos supor $0<\theta_{1}<\cdots<\theta_{g}<\pi$. Como $M \subset \mathbf{E}^{n+1}$ tem posto 2 seu grupo de Coxeter $W$ é um grupo diedral $* 2 g$. Logo $\theta_{i+1}-\theta_{i}=\frac{\pi}{g}$ ou seja

$$
\theta_{i}=\theta_{1}+\frac{i-1}{g} \pi
$$

para todo $i \in\{1, \ldots, g\}$. Além disso se $\mathbf{n}_{i}^{*}=\xi+\lambda_{i} P$ e $R_{i} \in W$ é a reflexão em relação a $\mathbf{n}_{i}^{\perp}=\mathbf{R n}_{i}^{*}$ temos que $R_{i} \mathbf{n}_{i}^{*} \in \mathbf{R n}_{i+2}^{*}$. Portanto da observação (2.3.2)

$$
m_{i}=m_{i+2},
$$

com índices congruentes módulo $g$.

Desta maneira temos mostrado o seguinte resultado.

Teorema 3.1.1 (Münzner). Seja $M \subset \mathbf{S}^{n}$ uma hipersuperfície completa da esfera com curvaturas principais $\cot \theta_{1}, \ldots, \cot \theta_{g}$ e $0<\theta_{1}<\cdots<\theta_{g}<\pi$. Então

$$
\theta_{i}=\theta_{1}+\frac{i-1}{g} \pi
$$

$e$

$$
m_{i}=m_{i+2},
$$

para todo $i \in\{1, \ldots, g-2\}$.

Agora, fazendo analogia com o feito na seção 2.1, consideraremos variedades paralelas e focais de hipersuperfícies da esfera unitária $\mathbf{S}^{n}$.

${ }^{*}$ Como $M \subset \mathbf{E}^{n+1}$ tem posto dois, $W^{p}$ contém pelo menos uma rotação diferente da identidade. Se $R \in W^{p}$ é uma rotação tal que seu ângulo correspondente $\vartheta$ (dado pela orientação $\left\{\xi_{p},-p\right\}$ ) é mínimo, dada uma reflexão $S \in W^{p}$, temos que $W^{p}$ é gerado por $R$ e $S$. Ainda, $\frac{\pi}{g}=\frac{\vartheta}{2}=\theta_{i+1}-\theta_{i}$, para todo $i \in\{1, \ldots, g-1\}$. 
Dada uma hipersuperfície $M \subset \mathbf{S}^{n}$ seja $\xi$ um campo normal unitário de $M$. Consideremos a aplicação $\phi_{t}=\cos t P+\sin t \xi$, onde $P$ é o campo vetor posição de $\mathbf{E}^{n+1}$ restrito a $\mathbf{S}^{n}$. Temos que

$$
d \phi_{t}=\cos t i d-\sin t A_{\xi}
$$

Dado $X \in \Gamma\left(E_{i}\right)$ tem-se

$$
d \phi_{t} X=\left(\cos t-\sin t \cot \theta_{i}\right) X=\frac{\sin \left(\theta_{i}-t\right)}{\sin \theta_{i}} X
$$

Suponhamos que existem $i, j \in\{1, \ldots, g\}$ e números inteiros $k_{i}, k_{j}$ tais que

$$
\theta_{j}+k_{j} \pi=t=\theta_{i}+k_{i} \pi
$$

Então

$$
\pi>\frac{|j-i|}{g} \pi=\left|\theta_{j}-\theta_{i}\right|=\left|k_{j}-k_{i}\right| \pi,
$$

assim $k_{j}=k_{i}$ e portanto $\theta_{j}=\theta_{i}$. Desta maneira o posto de $d \phi_{t}$ é $m=\operatorname{dim} M$ ou $m-m_{i}$ para algum $i \in\{1, \ldots, g\}$. Mais precisamente fazendo $M_{t}=\phi_{t}(M), M_{t} \subset \mathbf{S}^{n}$ é uma subvariedade e $\phi_{t}: M \longrightarrow M_{t}$ é uma submersão com

a. Se $t=\theta_{i}+k \pi$ ( $k$ inteiro), então o posto de $d \phi_{t}$ é $m-m_{i}$ e $T_{\phi_{t}(p)}=\oplus_{j \neq i} E_{j}(p)$.

b. Caso contrário $T_{\phi_{t}(p)}=T_{p} M$.

No primeiro caso dizemos que $M_{t}$ é uma variedade focal e no segundo que é uma variedade paralela de $M$. Dado $p \in M$ consideremos o vetor normal $\bar{\xi}_{\left.\phi_{(} p\right)}=\cos t-\sin t p$, então o operador de forma $\bar{A}$ de $M_{t}$ na direção de $\bar{\xi}_{\phi_{t}(p)}$ é da forma

$$
\bar{A}_{\bar{\xi}_{\phi_{t}(p)}}=\left(\sin t i d+\cos t A_{\xi_{p}}\right)\left(\cos t i d-\sin t A_{\xi_{p}}\right)^{-1},
$$

onde nos restringimos ao espaço horizontal. Assim os autovalores de $\bar{A}_{\bar{\xi}_{\phi_{t}}(p)}$ são da forma

$$
\bar{\lambda}_{j}=\cot \left(\theta_{j}-t\right)
$$


Logo as variedades focais $M_{i}=M_{\theta_{i}}$ de $M$ têm curvaturas principais da forma

$$
\cot \left(\theta_{j}-\theta_{i}\right)
$$

para todo $i \in\{1, \ldots, g\}$ com $j \neq i$.

Usaremos o teorema (3.1.1) para mostrar a fórmula fundamental de Cartan.

Proposição 3.1.2. Cada variedade focal $M_{i}$ de uma hipersuperfície da esfera é minimal.

Demonstração.

$$
\begin{aligned}
\operatorname{traço}\left(\bar{A}_{\bar{\xi}}\right) & =\sum_{j=1, j \neq i}^{g} m_{j} \cot \left(\theta_{j}-\theta_{i}\right) \\
& =\sum_{j=1}^{i-1} m_{j} \cot \left(\frac{(j-i) \pi}{g}\right)+\sum_{j=i+1}^{g} m_{j} \cot \left(\frac{(j-i) \pi}{g}\right) \\
& =\sum_{j=g-i+1}^{g-1} m_{j-(g-i)} \cot \left(\frac{(j-g) \pi}{g}\right)+\sum_{j=1}^{g-i} m_{j+i} \cot \left(\frac{j \pi}{g}\right) .
\end{aligned}
$$

Se $g$ é ímpar então todos os $m_{k}$ são iguais e se $g$ é par temos $m_{k}=m_{k-g}$ logo da equação acima segue que

$$
\begin{aligned}
\operatorname{traço}\left(\bar{A}_{\bar{\xi}}\right) & =\sum_{j=1}^{g-1} m_{j+i} \cot \left(\frac{j}{g} \pi\right) \\
& =-\sum_{j=1}^{g-1} m_{j+i} \cot \left(\pi-\frac{j}{g} \pi\right) \\
& =-\sum_{j=1}^{g-1} m_{j+i} \cot \left(\frac{g-j}{g} \pi\right) \\
& =-\sum_{j=1}^{g-1} m_{g-j+i} \cot \left(\frac{j}{g} \pi\right) .
\end{aligned}
$$

Novamente se $g$ é ímpar então todos os $m_{k}$ são iguais e se $g$ é par temos $(j+i)-(g-j+i)=g+2 j$ 
que é par, logo $m_{j+i}=m_{g-j+i}$. Portanto das duas últimas equações obtemos

$$
\operatorname{traço}\left(\bar{A}_{\bar{\xi}}\right)=-\operatorname{traço}\left(\bar{A}_{\bar{\xi}}\right)
$$

ou seja traço $\left(\bar{A}_{\bar{\xi}}\right)=0$. Isto é $M_{i}$ é minimal.

Corolário 3.1.3 (Fórmula fundamental de Cartan).

$$
\sum_{j \neq i} m_{j} \frac{1-\lambda_{i} \lambda_{j}}{\lambda_{i}-\lambda_{j}}=0
$$

\section{2 Órbitas da representação adjunta de um grupo de Lie compacto}

Sejam $G$ um grupo de Lie, equipado com uma métrica ai-invariante, $T \subset G$ um toro maximal e $\mathfrak{g}$ e $\mathfrak{t}$ suas respectivas álgebras de Lie. Denotemos com $\langle\cdot, \cdot\rangle,[\cdot, \cdot]$, e ad a forma de Killing o colchete de Lie e a ação adjunta de $\mathfrak{g}$, respectivamente. Consideremos a complexificação $\mathfrak{g}_{\mathbf{C}}$ de $\mathfrak{g}$, e denotemos com os mesmos símbolos acima as respectivas extensões canônicas destes objetos a $\mathfrak{g}_{\mathbf{C}}$, onde $\langle\cdot, \cdot\rangle$ é estendido a uma forma Hermitiana sobre $\mathfrak{g}_{\mathbf{C}}$. Para cada $Z \in \mathfrak{g} a d_{Z}$ é um operador anti-Hermitiano. Como $\mathfrak{t}$ é abeliana a família $\left\{a d_{Z}: Z \in \mathfrak{t}\right\}$ é comutativa e portanto simultaneamente diagonalizável sobre $\mathbf{C}$ com auto-valores imaginários puros, isto é; existe um conjunto finito $R$ de funcionais lineares não nulos $r: \mathfrak{t} \longrightarrow \mathbf{R}$, chamados raízes de $\mathfrak{g}$, e uma decomposição ortogonal única (salvo por uma permutação) de $\mathfrak{g}_{\mathbf{C}}$ da forma

$$
\mathfrak{g}_{\mathbf{C}}=\mathfrak{g}_{0} \oplus \bigoplus_{r \in R} \mathfrak{g}_{r}
$$

tal que $\mathfrak{g}_{r}$ é o auto-espaço associado ao auto-valor $\mathbf{i} r(Z)$, para todo $Z \in \mathfrak{t}$ e toda raiz $r \in R$, e $\mathfrak{g}_{0}$ é o auto espaço associado ao auto-valor nulo.

Proposição 3.2.1. Com a notação acima temos que

a. Se $r \in R$, então $-r \in R$. Assim

$$
R=\left\{r_{1}, \ldots, r_{s},-r_{1}, \ldots,-r_{s}\right\}
$$


onde $r_{j} \neq r_{k}$ se $j \neq k$ e $r_{j} \neq 0$, para todo par $j, k \in\{1, \ldots, s\}$. Logo

$$
\mathfrak{g}_{\mathbf{C}}=\mathfrak{g}_{0} \oplus \bigoplus_{j=1}^{s} \mathfrak{g}_{r_{j}} \oplus \mathfrak{g}_{-r_{j}} .
$$

b. $\operatorname{dim}_{\mathbf{C}} \mathfrak{g}_{r_{j}}=1$ e se $V_{j}=\left(\mathfrak{g}_{r_{j}} \oplus \mathfrak{g}_{-r_{j}}\right) \cap \mathfrak{g}$, então $\operatorname{dim}_{\mathbf{R}} V_{j}=2$, para todo $j \in\{1, \ldots, s\}$.

c. $\mathfrak{g}_{0}=\mathfrak{t} \oplus$ it.

Para uma demonstração destes fatos ver [DK99] capítulo 3.

Proposição 3.2.2. Na mesma via da proposição acima

a. Seja $r \in R, r=r_{j}$. Se $Y+\mathbf{i} X$ gera $\mathfrak{g}_{r}$ então $\{X, Y\}$ é uma base de $V_{j}$.

b. Temos que

$$
[Z, X]=r(Z) Y \quad e[Z, Y]=-r(Z) X,
$$

para todo $Z \in \mathfrak{t}$. E por tanto $X$ e $Y$ são ortogonais e têm o mesmo comprimento.

c. A matriz de $\left.A d_{\exp (Z)}\right|_{V_{j}}$ em relação à base ortonormal $\left\{\frac{1}{|X|} X, \frac{1}{|Y|} Y\right\}$ é

$$
\left[\begin{array}{cc}
\cos \left(r_{j}(Z)\right) & -\sin \left(r_{j}(Z)\right) \\
\sin \left(r_{j}(Z)\right) & \cos \left(r_{j}(Z)\right)
\end{array}\right]
$$

para todo $Z \in \mathfrak{t}$.

Demonstração. Sejam $X, Y \in \mathfrak{g}$ tais que $Y+\mathbf{i} X$ gera $\mathfrak{g}_{r}$, então $X+\mathbf{i} Y$ gera $\mathfrak{g}_{-r}$.

a. Seja $W \in V_{r}$ então $W=\lambda(Y+\mathbf{i} X)+\mu(X+\mathbf{i} Y), \operatorname{com} \lambda, \mu \in \mathbf{C}$. Como $W \in \mathfrak{g}$, então $\bar{W}=W$, assim

$$
W=\frac{1}{2}(W+\bar{W})=(\Re \mu-\Im \lambda) X+(\Re \lambda-\Im \mu) Y,
$$

onde $\Re$ e $\Im$ denotam a parte real e parte imaginária de um número complexo e $\bar{W}$ o vetor conjugado de $W$. Isto mostra que $X$ e $Y$ geram $V_{r}$, e como $\mathfrak{g}_{r} \cap \mathfrak{g}_{-r}=\{0\}$, então $X$ e $Y$ são linearmente independentes. 
b. Dado $Z \in \mathfrak{t}$ temos que

$$
\begin{aligned}
{[Z, Y]+\mathbf{i}[Z, X] } & =[Z, Y+\mathbf{i} X] \\
& =a d_{Z}(Y+\mathbf{i} X) \\
& =\mathbf{i} r(Z)(Y+\mathbf{i} X) \\
& =\mathbf{i} r(Z) Y-r(Z) X,
\end{aligned}
$$

$\operatorname{logo}([Z, Y]+r(Z) X)+\mathbf{i}([Z, X]-r(Z) X)=0$.

c. Dado $Z \in \mathfrak{t} \operatorname{com} r(Z) \neq 0$. Do item anterior tem-se

$$
\begin{aligned}
-r(Z)\langle X, X\rangle & =\langle[Z, Y], X\rangle \\
& =-\langle Y,[Z, X]\rangle \\
& =-r(Z)\langle Y, Y\rangle
\end{aligned}
$$

e

$$
\begin{aligned}
-r(Z)\langle X, Y\rangle & =\langle[Z, Y], Y\rangle \\
& =-\langle Y,[Z, Y]\rangle \\
& =r(Z)\langle Y, X\rangle
\end{aligned}
$$

d. Consideremos a base ortonormal $\left\{\frac{1}{|X|} X, \frac{1}{|Y|} Y\right\}$ do subespaço $V_{r}$. Dado $Z \in \mathfrak{t}$, dos dois itens anteriores segue que a matriz de $\left.a d_{Z}\right|_{V_{r}}$ em relação a esta base é

$$
\left[\begin{array}{cc}
0 & -r(Z) \\
r(Z) & 0
\end{array}\right]
$$

Da identidade exp oad $=A d \circ$ exp segue que a matriz de $\left.A d_{\exp (Z)}\right|_{V_{r}}$ em relação a esta base é

$$
\exp \left[\begin{array}{cc}
0 & -r(Z) \\
r(Z) & 0
\end{array}\right]=\left[\begin{array}{cc}
\cos r(Z) & -\sin r(Z) \\
\sin r(Z) & \cos r(Z)
\end{array}\right]
$$


Como conseqüência das duas proposições acima temos o seguinte resultado.

Corolário 3.2.3. Sejam $G$ um grupo de Lie conexo e compacto, $T \subset G$ um toro maximal e, $\mathfrak{g} e$ $\mathfrak{t}$ as álgebras de Lie de $G$ e $T$, respectivamente. Então existem subespaços $V_{j}$ e funcionais lineares distintos $r_{j}: \mathfrak{t} \longrightarrow \mathbf{R}, j \in\{1, \ldots, s\}$ tais que

a. $\mathfrak{g}$ tem uma única decomposição (salvo uma permutação) da forma

$$
\mathfrak{g}=\mathfrak{t} \oplus \bigoplus_{j=1}^{s} V_{j}
$$

b. Para toda métrica bi-invariante sobre $G$ existe uma base ortogonal de $V_{j}$ tal que a matriz de $\left.A d_{\exp (Z)}\right|_{V_{j}}$ nesta base de da forma

$$
\left[\begin{array}{cc}
\cos \left(r_{j}(Z)\right. & -\sin \left(r_{j}(Z)\right) \\
\sin \left(r_{j}(Z)\right) & \cos \left(r_{j}(Z)\right)
\end{array}\right],
$$

para todo $Z \in \mathfrak{t}$.

Sejam $G$ um grupo de Lie conexo e compacto, $T \subset G$ um toro maximal e, $\mathfrak{g}$ e $\mathfrak{t}$ as álgebras de Lie de $G$ e $T$, respectivamente. Suponhamos que $Z \in \mathfrak{t}$ é tal que a órbita $M=A d_{G} Z$ é principal. Então $r_{j}(Z) \neq 0$ para todo $j \in\{1, \ldots, s\}$. Dados $\xi, \xi^{\prime} \in \mathfrak{t}$ tem-se

$$
\left\langle\xi,\left[X, \xi^{\prime}\right]\right\rangle=-\left\langle\xi,\left[\xi^{\prime}, X\right]\right\rangle=\left\langle\left[\xi, \xi^{\prime}\right], X\right\rangle=\langle 0, X\rangle=0
$$

para todo $X \in \mathfrak{g}$. De outro lado

$$
T_{Z} M=\{[X, Z]: x \in \mathfrak{g}\} .
$$

Assim da equação $(3.4), \nu_{Z} M=\mathfrak{t}$. Dado $\xi \in \mathfrak{t}$ consideremos o campo normal $\hat{\xi}$ dado por

$$
\hat{\xi}_{A d_{g} Z}=A d_{g} \xi
$$

para todo $g \in G$. Diferenciando $\hat{\xi}$ em relação a $g$ obtemos

$$
d \hat{\xi}_{Z}[X, Z]=a d_{X} \xi=[X, \xi] .
$$

Pela equação (3.4) $\hat{\xi}$ é uma campo normal paralelo globalmente definido sobre $M$. Portanto $M$ tem 
fibrado normal raso. Além disso

$$
A_{\hat{\xi}}[X, Z]=-[X, \xi] .
$$

Se $X_{j}$ e $Y_{j}$ são como na equação (3.3) obtemos

$$
A_{\hat{\xi}} X_{j}=-\frac{r_{j}(\xi)}{r_{j}(Z)} X_{j}
$$

$$
A_{\hat{\xi}} Y_{j}=-\frac{r_{j}(\xi)}{r_{j}(Z)} Y_{j}
$$

assim $M$ tem curvaturas principais constantes $\lambda_{j}(\hat{\xi})=-\frac{r_{j}(\xi)}{r_{j}(Z)}$ ao longo de $\hat{\xi}$ e distribuições de curvatura $E_{j}=V_{j}$ com $m_{j}=2$, para $j \in\{1, \ldots, s\}$. Portanto $M$ é isoparamétrica com normais de curvatura

$$
\mathbf{n}_{j}(Z)=\frac{-1}{\left\langle Z_{j}, Z\right\rangle} Z_{j}
$$

onde $Z_{j}$ está definido por $r_{j}\left(Z^{\prime}\right)=\left\langle Z_{j}, Z^{\prime}\right\rangle$, para todo $Z^{\prime} \in \mathfrak{t}$ e todo $j \in\{1, \ldots, s\}$. 


\section{APÊNDICE A}

\section{Pontos críticos de funções distância e subvariedades isoparamétricas}

Neste apêndice pretendemos mostrar algumas propriedades das subvariedades isoparamétricas do espaço Euclidiano que podem ser desenvolvidas usando teoria de pontos críticos. Esta ferramenta é usada na teoria de subvariedades isoparamétricas de espaços de Hilbert (vide [PT88]). O teorema (2.6.2) tem varias conseqüências, entre elas que a intersecção do espaço normal afim a um ponto de uma subvariedade isoparamétrica do espaço Euclidiano intersecta à subvariedade exatamente na órbita do ponto dada pela ação do grupo de Coxeter. Neste capítulo daremos outra prova deste fato e mostraremos outros resultados interessantes como a proposição (A.4) e o corolário (A.5). Para isto estudaremos algumas propriedades da função distância Euclidiana a um ponto fixo restrita a uma subvariedade isoparamétrica. Seria necessário desenvolver alguns conceitos topológicos, mais precisamente subvariedades taut. Não faremos isto e para simplificar enunciaremos sem demonstração a proposição (A.1), este é um caso particular de [PT88] corolário (8.3.3).

Neste capítulo $f_{c}$ denotara a restrição a $M$ da função $d_{c}$ dada por

$$
d_{c}(x)=\frac{1}{2}|x-c|^{2},
$$


para todo $x \in \mathbf{E}^{n}$, onde $c \in \mathbf{E}^{n}$ é fixo e $M \subset \mathbf{E}^{n}$ é uma subvariedade isoparamétrica.

Proposição A.1. Seja $M \subset \mathbf{E}^{n}$ uma subvariedade isoparamétrica conexa. Consideremos o conjunto $M_{r}\left(f_{c}\right)=f_{c}^{-1}([0, r])$, onde $r$ é um número real não negativo. Então $M_{r}\left(f_{c}\right)$ e conexo.

Para uma demonstração deste fato vide [PT88] capítulo 8.

Lema A.2. $p \in M$ é ponto crítico de $f_{c}$ se e somente se $c \in p+\nu_{p} M$ e neste caso

$$
\text { Hess } f_{c}(p)\left(u, u^{\prime}\right)=\left\langle\left(i d_{T_{p} M}-A_{c-p}\right) u, u^{\prime}\right\rangle
$$

para todo par de vetores $u, u^{\prime} \in T_{p} M$, onde Hess $f_{c}$ denota o Hessiano de $f_{c}$.

Demonstração. Dado $p \in M$, o gradiente de $f_{c}$ em $p, \operatorname{grad} f_{c}(p)$, está dado por

$$
\left\langle\operatorname{grad} f_{c}(p), u\right\rangle=\langle p-c, u\rangle
$$

para todo $u \in T_{p} M$. Daqui segue a primeira afirmação. Agora sejam $X, Y \in \Gamma(T M)$ então

$$
\operatorname{grad} d_{c}=\operatorname{grad} f_{c}+\left(\operatorname{grad} d_{c}\right)^{\perp}
$$

e assim

$$
\text { Hess } \begin{aligned}
f_{c}(X, Y) & =\left\langle\nabla_{X} \operatorname{grad} f_{c}, Y\right\rangle \\
& =\left\langle\bar{\nabla}_{X} \operatorname{grad} f_{c}, Y\right\rangle \\
& =\left\langle\bar{\nabla}_{X} \operatorname{grad} d_{c}, Y\right\rangle-\left\langle\bar{\nabla}_{X}\left(\operatorname{grad} d_{c}\right)^{\perp}, Y\right\rangle \\
& =\operatorname{Hess}_{c}(X, Y)+\left\langle A_{\left(\operatorname{grad}_{c}\right)^{\perp}} X, Y\right\rangle \\
& =\langle X, Y\rangle+\left\langle A_{\left(\operatorname{grad}_{c}\right)^{\perp}} X, Y\right\rangle .
\end{aligned}
$$

Portanto tem-se (A.1).

Lema A.3. Seja $p \in M$, então

a. $W^{p}(p) \subset M$ e p $+\nu_{p} M=q+\nu_{q} M$ para todo $q \in W^{p}(p)$.

b. Se p é ponto crítico de $f_{c}$ então $q$ é ponto crítico de $f_{c}$ para todo $q \in W^{p}(p)$. 
Demonstração. Com efeito,

a. Seja $\rho=\rho_{i_{1}}^{p} \cdots \rho_{i_{s}}^{p} \in W^{p}$. Dos lemas (2.3.1) (item a.) e (2.3.3) segue que

$$
\rho(p)=a_{\sigma_{i_{1}} \cdots \sigma_{i_{s-1}}\left(i_{s}\right)} \circ a_{\sigma_{i_{2}} \cdots \sigma_{i_{s-1}}\left(i_{s}\right)} \circ \cdots \circ a_{\sigma_{i_{s-1}}\left(i_{s}\right)} \circ a_{i_{s}}(p) .
$$

Se $\xi_{i}=\frac{1}{\left|\mathbf{n}_{i}\right|^{2}} \mathbf{n}$, sabemos que $a_{i}(M)=M_{\xi_{i}}$, para $i \in\{1, \ldots, g\}$, então

$$
\begin{aligned}
a_{\sigma_{i_{1}} \cdots \sigma_{i_{s-1}}\left(i_{s}\right)} \circ a_{\sigma_{i_{2}} \cdots \sigma_{i_{s-1}}\left(i_{s}\right)} \circ \cdots \circ a_{\sigma_{i_{s-1}}\left(i_{s}\right)} \circ a_{i_{s}}(M) & \\
& =\left(\cdots\left(\left(M_{\xi_{i_{s}}}\right) \xi_{i_{s-1}}\right) \cdots\right)_{\xi_{i_{1}}} \\
& =M .
\end{aligned}
$$

Portanto $q=\rho(p) \in M$ e $p+\nu_{p} M=q+\nu_{q} M$.

b. Suponhamos que $p$ é ponto crítico de $f_{c}$. Dado $q \in W^{p}(p)$, do item a. segue que $p+\nu_{p} M=q+\nu_{q} M$.

Logo $c \in q+\nu_{q} M$ e do lema acima, $q$ é um ponto crítico de $f_{c}$.

Proposição A.4. Sejam $p \in M$ e $C_{p}$ a câmera de Weyl de $p+\nu_{p} M$ contendo $p$. Se $x_{0} \in C_{p}$, então

a. $p$ é um ponto crítico de $f_{c}$ de índice 0 .

b. $f_{c}(p)$ é mínimo global de $f_{c}$ em $M$

c. Se $c \notin F_{M}(p)$ então $f_{c}^{-1}(f(p))$.

Demonstração. a. Temos que $\Delta_{p}^{+}(p)=\left\{\frac{-1}{\left|\mathbf{n}_{i}(p)\right|} \mathbf{n}_{i}(p): i=1, \ldots, g\right\}$, assim

$$
1-\left\langle\mathbf{n}_{i}(p), c-p\right\rangle \geq 0,
$$

para todo $i \in\{1, \ldots, g\}$. Da equação (A.1) tem-se

$$
\text { Hess } f_{c}(p)(u, u) \geq 0
$$

se $u \in T_{p} M$ com $u \neq 0$. Logo $p$ é um ponto crítico de índice 0 . 
b. Para $0<t<1$ consideremos as funções $f_{t}=f_{c_{t}}$, onde $c_{t}=c+t(p-c)$. Então $p$ é um ponto crítico de $f_{t}$ e pela equação (A.2) temos que

$$
1-\left\langle\mathbf{n}_{i}(p),(1-t)(c-p)\right\rangle>0,
$$

para todo $i \in\{1, \ldots, g\}$. Usando de novo a equação (A.1) obtemos

$$
\text { Hess } f_{t}(p)(u, u)>0
$$

para $u \in T_{p} M$, com $u \neq 0$. Segue que $p$ é um ponto crítico não degenerado de índice 0 de $f_{t}$; ainda mais, $p$ é um ponto de mínimo local de $f_{t}$ em $M$ e portanto é isolado. Seja $\mathcal{A}_{t}$ uma vizinhança de $p$ em $M$ tal que $p$ é ponto de mínimo global de $\left.f_{t}\right|_{\mathcal{A}_{t}}$. Fazendo $r_{t}=f_{t}(p)$, temos que $\mathcal{A}_{t} \cap M_{r_{t}}\left(f_{t}\right)=\{p\}$, logo $\{p\}$ é aberto e fechado em $M_{r_{t}}\left(f_{t}\right)$. Do lema (A.1) $M_{r_{t}}\left(f_{t}\right)$ é conexo, então $M_{f_{t}(p)}\left(f_{t}\right)=\{p\}$. Isto é

$$
\left|x-c_{t}\right| \geq\left|p-c_{t}\right|
$$

para todo $x \in M$. Fazendo $t \rightarrow 0, f_{c}(p)$ é um ponto de mínimo global de $f$ em $M$.

c. Do item b. temos que $p \in f_{c}^{-1}\left(f_{c}(p)\right) \subset M_{f_{c}(p)}(f)$. Agora, se $c \notin F_{M}(p)$ então na equação (A.2) a desigualdade é estrita. Assim, usando os mesmos argumentos acima, $M_{f_{c}(p)}(f)=\{p\}$.

Corolário A.5. Sejam $p, q \in M$. Se $c \in\left(p+\nu_{p} M\right) \cap\left(q+\nu_{q} M\right)$ então $c \notin F_{M}(p)$ se e somente se $c \notin F_{M}(q)$, e neste caso $p \in W^{q}(q)$.

Demonstração. Pela proposição (2.3.10) existem $p^{\prime} \in W^{p}(p)$ e $q^{\prime} \in W^{q}(q)$ tais que $c \in C_{p^{\prime}} \cap C_{q^{\prime}}$. Do item b. da proposição anterior $f_{c}\left(p^{\prime}\right)=f_{c}\left(q^{\prime}\right)$ é mínimo global de $f_{c}$ em $M$. Se $c \notin F_{M}(p)=F_{M}\left(p^{\prime}\right)$, pelo item c. da mesma proposição $p^{\prime}=q^{\prime}$. Logo $c \notin F_{M}\left(q^{\prime}\right)=F_{M}(q)$ e $p \in W^{p^{\prime}}\left(p^{\prime}\right)=W^{q^{\prime}}\left(q^{\prime}\right)=$ $W^{q}(q)$.

Proposição A.6. $M \cap\left(p+\nu_{p} M\right)=W^{p}(p)$.

Demonstração. Do item a. do lema (A.3) $W^{p}(p) \subset M \cap\left(p+\nu_{p} M\right)$. Agora, se $c \in M \cap\left(p+\nu_{p} M\right)$ então $c \in\left(x_{0}+\nu_{c} M\right) \cap\left(p+\nu_{p} M\right)$. Como $c \notin F_{M}(c)$, do corolário acima $c \in W^{p}(p)$. 
[BCO03] J. Berndt, S. Console, and C. Olmos. Submanifolds and holonomy. volume 434 of Research Notes in Mathematics. Chapman \& Hall/CRC, Boca Raton-London-New YorkWashington,D.C., 2003.

[BG70] C.T. Benson and L.C. Grove. Finite reflection groups. Bogden \& Quigley, INC. publishers, Tarrytown-on-Hodson, New York-Belmont, California, one edition, 1970.

[Bro88] K.S. Brown. Buldings. Springer-Verlag, Berlin-Heidelberg-New York, one edition, 1988.

[CCJ07] T.E. Cecil, Q.S Chi, and G.R. Jensen. Isoparametric hypersurfaces whit four principal curvatures. Ann. of Math., 166:1-76, 2007.

[DK99] J.J. Duistermaat and J.A.C. Kolk. Lie groups. Springer-Verlag, Berlin-Heidelberg-New York, one edition, 1999.

[HL97] E. Heintze and X. Liu. A splitting theorem of isoparametric submanifolds in Hilbert space. J. Differential Geom, 45:319-335, 1997.

[HL99] E. Heintze and X. Liu. Homogeneity of infinite dimensional isoparametric submanifolds. Annals of Mathematics, 149:149-181, 1999. 
[Nom73] K. Nomizu. Characteristic roots and vectors of a differentiable family of symmetric matrices. Linear and Multilinear Algebra, 1:159-162, 1973.

[PT88] R.S. Palais and C.L. Terng. Critical point theory and submanifold geometry. volume 1353 of Lecture Notes in Mathematics. Springer-Verlag, Berlin-Heidelberg-New York-LondonParis-Tokyo, 1988.

[Rec79] H. Reckziegel. On the eigenvalues of the shape operator of an isometric immersion into a space of constant curvature. Math. Ann., 243:71-82, 1979.

[Spi70] M. Spivak. A comprehensive introduction to differential geometry, volume 1. Publish or Perish, third edition, 1970.

[Tho00] Gudlagur Thorbergsson. Handbook of differential geometry, volume 1, chapter 10. Elsevier Science B.V., one edition, 2000. 
afim

transporte paralelo, 32

aplicação isoparamétrica, 44

câmera de Weyl, 38

campo

básico, 18

horizontal, 18

projetável, 18

vertical, 18

curvatura média

campo de, 15

função de, 15

curvatura principal, 14

constante, 21

na direção de $v, 14$

distribuição

auto-paralela, 15

de curvatura, 14

de nulidade, 14

horizontal, 18

vertical, 18 domínio fundamental, 38

equação

de Codazzi, 10

de Gauss, 10

de Ricci, 10

fórmula

fundamental de Cartan, 53

de Gauss, 8

de Weingarten, 9

focal

conjunto, 25

hiperplano, 32

ponto, 22

variedade, 22

grupo

de Coxeter, 31

grupo de Coxeter

de $M$ em $p, 35$

levantamento horizontal, 18 
normais de curvatura, 26

normal

campo, 7

espaço, 7

fibrado, 6

primeiro espaço, 16

projeção, 6

tensor de curvatura do fibrado, 10

operador de forma, 7

na direção de $\xi, 7$

parâmetro diferencial

primeiro, 1

segundo, 1

posto

de $M, 31$

do grupo de Coxeter, 31

raso

fibrado normal, 10, 21

Riemanniana

submersão, 18

segunda forma fundamental, 7

subvariedade

isoparamétrica, 21

mínima, 15

substancial, 16

totalmente geodésica, 15

totalmente umbílica, 15

variedade paralela, 22 